\title{
TIGHT CLOSURE, INVARLANT THEORY, AND THE BRIANÇON-SKODA THEOREM
}

\author{
MELVIN HOCHSTER AND CRAIG HUNEKE
}

\section{CONTENTS}

1. Introduction

2. Notation and terminology

3. The definition of tight closure for ideals in characteristic $p$ and in characteristic 0

4. Basic properties of tight closure for ideals in characteristic $p$

5. Integral closure and the Briançon-Skoda theorem in characteristic $p$

6. Test elements

7. Constraints on systems of parameters in characteristic $p$

8. Tight closure for submodules in characteristic $p$

9. Phantom homology and the phantom acyclicity criterion in characteristic $p$

10. The syzygy theorem and variant notions of tight closure in characteristic $p$

11. Phantom acyclicity for images of Gorenstein rings

\section{INTRODUCTION}

Throughout this paper all rings are commutative, with identity, and Noetherian, unless otherwise specified. We introduce the notion of the tight closure of an ideal in prime characteristic $p$ and for algebras essentially of finite type over a field of characteristic 0 . Later, we extend the theory to submodules of modules. As a consequence, we are able to give new proofs, which are remarkably simple in characteristic $p$ (often just a few sentences), of several results that were not thought to be particularly related, i.e., that rings of invariants of linearly reductive groups acting on regular rings are Cohen-Macaulay, that the integral closure of the $n$th power of an $n$ generator ideal of a regular ring is contained in the ideal (the Briançori-Skoda theorem), of the monomial conjecture, and of the syzygy theorem.

(Cf. [HR1, B, Ke, and Bor] for the background on invariant theory, [BrS, Sk, LS, and LT] for information about the Briançon-Skoda theorem, and [Wal] for the question of J. Mather that initially motivated the work of Briançon and

Received by the editors December 19, 1988.

1980 Mathematics Subject Classification (1985 Revision). Primary 13E05, 13B20, 13C99, $13 \mathrm{D} 99,13 \mathrm{H} 99$.

Both authors were supported in part by grants from the National Science Foundation. 
Skoda. Cf. [PS1, Ho3, Ho4, Ho9, Ro1, Ro5, Ro6, Du2, EvG1, and EvG2] for more about the monomial conjecture, the syzygy theorem, and the other local homological conjectures.)

The new proofs yield much more general theorems. For example, we shall show in [HH7] that if $S$ is any Noetherian regular ring containing a field and $R$ is a direct summand of $S$ as an $R$-module (we shall sometimes say, briefly, that $R$ is a summand of $S$ to describe this situation: we always mean $R \rightarrow S$ is $R$-split), then $R$ is Cohen-Macaulay. This result was not previously known in this generality. Moreover, this illustrates a general principle. The results proved here using tight closure techniques but which do not refer specifically to tight closure can be extended to the general equicharacteristic case by ring Artin approximation to reduce to a situation in which tight closure is defined.

One of the most important characteristics of tight closure is that in a regular ring every ideal is tightly closed. We call the Noetherian rings all of whose localizations have this property "F-regular." This is an important class of rings that includes the rings of invariants of linearly reductive groups acting on regular rings. A key point is that if $S$ is F-regular and $R$ is a direct summand of $S$ as an $R$-module, then $R$ if F-regular. It turns out that, under mild conditions (like being a homomorphic image of a Cohen-Macaulay ring or a weakening of the requirements for excellence), F-regular rings, which are always normal, are Cohen-Macaulay as well. One of our objectives in the sequels to this paper is to explore the theory of these rings, including its connection with the theory of rational singularities. (The $F$ stands for Frobenius. The reason for this usage will become clear once tight closure is defined.)

Suppose, for simplicity, that $R$ is local. One of the characterizations of the Cohen-Macaulay (C-M) property for $R$ is that for elements $x_{1}, \ldots, x_{n}$ that are part of a system of parameters, $\left(x_{1}, \ldots, x_{n-1}\right) R:{ }_{R} x_{n}=\left(x_{1}, \ldots, x_{n-1}\right) R$. (Recall that $I:{ }_{R} x=I: x=\{r \in R: x r \in I\}$, the pullback of the annihilator of $x$ in $R / I$ to $R$.) A critically important property of tight closure is that, under mild conditions on $R,\left(x_{1}, \ldots, x_{n-1}\right): x_{n} \subseteq\left(x_{1}, \ldots, x_{n-1}\right)^{*}$. Of course, we are not assuming that $R$ is C-M. We describe this property briefly by saying "the tight closure captures colons." This gives an important form of control of the cohomological obstruction to the C-M property. (Note that whenever the tight closure captures colons and $R$ is F-regular, $R$ must be C-M.) What is more, there is a similar result that is global and applies to a sequence of operations (such as intersection, colon, sum, and product) on ideals generated by monomials in elements that are locally parameters. There is an "expected" answer, which is correct in the case where the $x_{i}$ 's form an $R$-sequence, and the correct answer, in general, is in the tight closure of the "expected" answer. See $\S 7$.

Suppose that $R \subseteq S$ are domains for which tight closure is defined, and $S$ is regular (or F-regular). Another important point is that for any ideal $I \subseteq R$, $I^{*} S=I S$. In particular, the tight closure of an ideal $I$ in a domain $R$ is contained in the contraction to $R$ if $I S$ for every (F-)regular domain $S \supseteq R$. In consequence, the tight closure of an ideal is contained in the integral closure, but is usually much smaller. 
Combining this result with the preceding paragraph, one can show that, under mild conditions on the equicharacteristic ring $R$, if $x_{1}, \ldots, x_{n}$ are locally parameters in $R$ and $R \subseteq S$, where $S$ is regular, then $\left(\left(x_{1}, \ldots, x_{n-1}\right):{ }_{R} x_{n}\right) S=$ $\left(x_{1}, \ldots, x_{n-1}\right) S$, a very surprising result.

Here is an example of a theorem proved using tight closure for submodules that is closely allied to the result on colons cited just above.

Theorem (Vanishing Theorem). Let $A \subseteq R \subseteq S$ be excellent equicharacteristic rings such that $A, S$ are regular domains and $R$ is module-finite over $A$. Let $M$

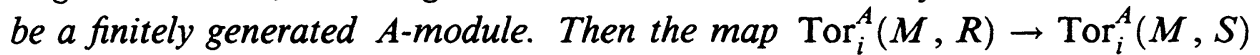
is 0 for all $i \geq 1$.

We have not attempted to state the most general version of this result here. Improved versions can be derived from the phantom acyclicity criterion of $\S 9$ of this paper, and this will be carried through in [HH4]. But we note that the version given already implies that direct summands of regular rings are C-M (using only the case $M=K$ ) and the direct summand (or monomial) conjecture (using only the case where $S$ is a discrete valuation ring). The proof uses Artin approximation to reduce to a case where tight closure techniques can be employed. One shows that appropriate boundaries are in the tight closure of the cycles, which makes the homology vanish after one maps to a regular ring.

The structure of the manuscript is as follows. In the next section, we give some important notation and conventions. In $\S 3$ we discuss the basic definitions for tight closure for ideals. We felt it worthwhile to include the characteristic 0 definitions for ideals, although the detailed study of characteristic 0 is postponed until [HH7]. In $\S 4$ we establish the basic properties for tight closure in the case of ideals and prove that direct summands of regular rings are Cohen-Macaulay in characteristic $p . \S 5$ deals with results related to the Briançon-Skoda theorem.

In $\S 6$ we introduce the notion of a test element. In certain circumstances, one can show that fixed elements of the ring can be used in all tight closure tests. In $\S 7$ we study operations on ideals generated by parameters and prove results about when they are contained in the tight closure of the answer one would expect if they formed an $R$-sequence. The next section introduces the notion of tight closure for submodules in characteristic $p$ and establishes many basic properties.

In $\S 9$ we introduce the notion of phantom homology. This occurs when the cycles in a module of a complex are in tight closure, within that module, of the boundaries. We give a criterion, referred to as the "phantom acyclicity criterion," for a free complex along with all its images under iterations of the Frobenius functor to have higher phantom homology.

In $\S 10$ we explore various alternative notions of tight closure and use one of them to give a new proof of the syzygy theorem. In $\S 11$ we refine our phantom acyclicity criteria in the case where the ring is a homomorphic image of a Gorenstein ring of finite Krull dimension. The present paper ends with $\S 11$, but we want to describe briefly the planned contents of some sequels to this paper.

The techniques discussed here are still insufficient to deal with the case of rings that are not locally equidimensional. In [HH4] we solve that problem by 
using the notion of minheight and the related notion of being weakly CohenMacaulay, which are introduced in [HH4], as well as generalizing certain phantom acyclicity results to the case of complexes that are not necessarily free. These results are then applied in [HH4] to prove numerous vanishing theorems for maps of homology and cohomology that may be thought of as enormous generalizations of the Vanishing Theorem (for maps of Tor) discussed above.

In [HH4] we also study the notion of regular closure, which can be used as a substitute for tight closure in characteristic 0 . In a Noetherian domain $R$, an element is in the regular closure of an ideal if it is in the contraction back to $R$ of the expansion of the ideal to every regular domain that contains $R$. The tight closure is contained in the regular closure (which is obviously contained in the integral closure, where one uses contracted expansions from discrete valuation rings instead). Every theorem on tight closure in characteristic $p$ has a weaker version expressed in terms of regular closure which has the advantage of being capable of generalization to the equal characteristic 0 case. The generalizations are carried out in [HH7]. It should be noted that we do not know whether, when tight closure is defined, it coincides with the regular closure. Regular closure has the advantage of being defined much more generally. However, we cannot prove any interesting results about it directly. What we know is a consequence of tight closure techniques.

When $R$ is not a domain, one considers contracted expansions for maps to regular domains whose kernels are minimal primes. There is also a notion of regular closure for submodules of a module that plays a similar role when one studies tight closure for submodules.

In [HH4, Theorem 6.2] we give a new proof of a result of Roberts used in his demonstration of the new intersection theorem in mixed characteristic (see [Ro5] and [Ro6]) as well as a new proof of a greatly strengthened form of the improved new intersection theorem in characteristic $p$ using phantom acyclicity techniques. This result is generalized to the equal characteristic 0 case in [HH7].

In [HH5] we investigate rings in which every ideal generated by parameters is tightly closed. These are called F-rational by Fedder and Watanabe [FeW] and may coincide with rings with rational singularities in the affine equicharacteristic 0 case. They coincide with F-regular rings in the Gorenstein case, and this fact is used to prove some results on behavior of F-regular Gorenstein rings. This theory has the important corollary that a Gorenstein local ring of characteristic $p$ is F-regular provided that one ideal generated by a system of parameters is tightly closed.

In [HH5] we discuss the notion of a strongly F-regular ring. This class of rings is in many ways better behaved than the class of F-regular rings, but the notion is defined only for reduced rings $R$ of characteristic $p$ such that $R^{1 / p}$ is module-finite over $R$. In the Gorenstein case, when strong F-regularity is defined, it coincides with F-regularity and weak F-regularity. See also [HH3]. [HH5] also gives a further treatment of test elements and discusses the behavior of tight closure under smooth base change.

In [HH6] we discuss the notion of a phantom extension, which can be used to give a new proof of several local homological conjectures in characteristic $p$, 
including the existence of big Cohen-Macaulay modules and strengthenings in various directions of the direct summand conjecture. We also study a number of splitting problems. We show that weakly F-regular rings are direct summands of all module-finite extensions in characteristic $p$ and that the converse holds for locally excellent Gorenstein domains. One tool introduced in studying the converse is a sort of "Artin-Schreier closure" of an ideal. We give a generalization of the Briançon-Skoda theorem for the case of an isolated singularity whose statement does not refer to tight closure.

In [HH7], we develop tight closure theory and phantom homology theory in the equicharacteristic zero case, and study connections with rational singularities in characteristic zero.

Tight closure ideas are used in [HH8] to prove some surprising splitting theorems for cyclic $R$-modules generated by elements of small order in module-finite extension algebras of an F-regular ring $R$.

In [HH9], ideas closely connected with our study of Artin-Schreier closures of ideals in [HH6], with the theory of test elements for tight closure, and with the notion of Cohen-Macaulay tight closure discussed in $\S \S 10$ and 11 of this paper are used to prove the following result.

If $R$ is an excellent, semilocal, biequidimensional domain of characteristic $p$, then the integral closure $R^{+}$of $R$ in an algebraic closure of its fraction field is a big Cohen-Macaulay module for $R$ (every system of parameters for $R$ in its Jacobson radical is a regular sequence in $R^{+}$). Although the idea of the proof of the main result in [HH9] evolved from tight closure theory, the paper has been written so as to make it largely independent of the other tight closure papers.

Parts of this manuscript have been discussed in the announcement [HH1], in the expository papers [HH2] and [Hu2], and in [HH3].

\section{NOTATION AND TERMINOLOGY}

Unless otherwise specified, $A, R$, and $S$ denote Noetherian commutative rings with 1 . By a local ring we always mean a Noetherian ring with a unique maximal ideal. $R^{\circ}$ denotes the complement of the union of the minimal primes of $R . I$ and $J$ always denote ideals. Thus, "given $I \subseteq R$ " means given an ideal $I$ in the Noetherian ring $R$. Unless otherwise specified, given modules $M$ and $N$ are assumed to be finitely generated.

(2.1) Definition. We say that elements $x_{1}, \ldots, x_{n}$ in a Noetherian ring $R$ are parameters if for every prime ideal $P$ containing them, their images in $R_{P}$ are part of a system of parameters (s.o.p.).

Note that, with this definition, elements that generate the unit ideal are parameters. Since no prime contains them all, the condition holds vacuously.

We recall that $R$ is said to be a pure subring of $S$ (or that $R \rightarrow S$ is pure) if for every $R$-module $W$, the induced map of $R \otimes_{R} W \rightarrow S \otimes_{R} W$ is injective (of course, the first module is simply $W$ ).

If $R$ is Noetherian, this is equivalent to assuming that $R$ is a direct summand, as an $R$-module, of every finitely generated $R$-submodule of $S$ containing $R$. In particular, it suffices that $R$ be a direct summand of $S$ as an 
$R$-module. If $R$ is a complete local ring, then $R \subseteq S$ is pure iff $R$ is a direct summand of $S$ as an $R$-module. See [HR1] and [HR2].

We make the following notational conventions in discussing characteristic $p$. We shall always use $p$ to denote a positive prime integer. We shall use $e$ for a variable element of $\mathbf{N}$, the set of nonnegative integers, and $q$ for a variable element of the set $\left\{p^{e}: e \in \mathbf{N}\right\}$. Thus, "for all $e^{"}$ is synonymous with "for all $e \in \mathbf{N}$ " while "for some $q$ " is synonymous with "for some $q$ of the form $p^{e}$ with $e \in \mathbf{N}$."

If $R$ is reduced of characteristic $p$, we write $R^{1 / q}$ for the ring obtained by adjoining all $q$ th roots of elements of $R$. The inclusion map $R \subseteq R^{1 / q}$ is isomorphic with the map $F^{e}: R \rightarrow R$, where $q=p^{e}, F$ is the Frobenius endomorphism of $R$, and $F^{e}$ is the $e$ th iteration of $F$, i.e., $F^{e}(r)=r^{q}$. When $R$ is reduced, we write $R^{\infty}$ for the $R$-algebra $\bigcup_{q} R^{1 / q}$. Note that $R^{\infty}$ is a chronic exception to the rule that the rings we consider be Noetherian. When $R$ is a reduced Noetherian ring of characteristic $p$, we use $\left(R^{\infty}\right)^{\circ}$ for the complement of the union of the minimal primes of $R^{\infty}:\left(R^{\infty}\right)^{\circ}=\bigcup_{q}\left(R^{1 / q}\right)^{\circ}$.

If $I \subseteq R$ and $q=p^{e}$, then $I^{[q]}$ denotes $\left(i^{q}: i \in I\right)=F^{e}(I) R$. If $S$ generates $I$, then $\left\{i^{q}: i \in S\right\}$ generates $I^{[q]}$.

\section{THE DEFINITION OF TIGHT CLOSURE FOR IDEALS IN CHARACTERISTIC $p$ AND IN CHARACTERISTIC 0}

In this section we give briefly the definitions of tight closure for both ideals in characteristic $p$ and for algebras finitely generated over a field in characteristic 0 . We also give a definition for algebras essentially of finite type over a field of characteristic 0 .

(3.1) Definition. Let $I \subseteq R$ of characteristic $p$ be given. We say that $x \in I^{*}$, the tight closure of $I$, if there exists $c \in R^{\circ}$ such that $c x^{q} \in I^{[q]}$ for all $q \gg 0$, i.e., for all sufficiently large $q$ of the form $p^{e}$. If $I=I^{*}$, we say that $I$ is tightly closed.

(3.2) Remarks. Note that if $R$ is a domain, which is by far the most important case, the condition that $c \in R^{\circ}$ is simply the condition that $c$ not be 0 . Note also that if $R$ is reduced, then $c x^{q} \in I^{[q]}$ iff $c^{1 / q} x \in I R^{1 / q}$. Thus, if $x \in I^{*}$, then for some $c \in R^{\circ}$, we have that $c^{1 / q} x \in I R^{\infty}$ for all $q$ (this condition gets stronger as $q$ gets larger). This gives a heuristic argument for regarding $x$ as being "nearly" in $I$, or, at least, $I R^{\infty}$. It is multiplied into $I R^{\infty}$ by elements that, in a formal sense, are getting "closer and closer" to 1 (since $1 / q \rightarrow 0$ as $q \rightarrow \infty)$. See Remark (6.12) for an additional perspective on tight closure.

In a preliminary version of this manuscript a slightly different definition of tight closure was used. It was required that $c x^{q} \in I^{[q]}$ for all $q$ instead of all sufficiently large $q$. This change does not affect what $I^{*}$ turns out to be when $I$ has positive height or when $R$ is reduced (cf. Proposition (4.1)(c)) and yields a more satisfactory theory in other cases.

(3.3) Definition. Let $R$ be a finitely generated algebra over a field $K$ of characteristic $0, I \subseteq R$, and $x, c \in R$. We call a triple $\left(D, R_{D}, I_{D}\right)$ descent data 
for $R, I, x$, and $c$ if $D$ is a finitely generated Z-subalgebra of $K, R_{D}$ is a finitely generated $D$-subalgebra of $R$, and $I_{D}$ is an ideal of $R_{D}$ such that

(a) $I_{D}$ and $R_{D} / I_{D}$ are $D$-free.

(b) The canonical map $K \otimes_{D} R_{D} \rightarrow R$ induced by the inclusions of $K$ and $R_{D}$ in $R$ is a $K$-algebra isomorphism.

(c) $I=I_{D} R$.

(d) $x, c \in R_{D}$.

(3.4) Remarks. Condition (a) also implies that $R_{D}$ is $D$-free. Note that if we are given $D, R_{D}$, and $I_{D}$ such that all conditions but (a) are satisfied, we can achieve (a) by replacing $D, R_{D}, I_{D}$ by their tensor products with $D_{a}$ for a suitable nonzero element $a \in D$, by the lemma of generic flatness (cf. [Mat, Chapter $8, \S 22$, p. 156] or [HR1, $\S 8$, p. 146]). When $L$ is a $D$-algebra, we use the subscript $L$ to denote objects and images after applying $L \otimes_{D}$. If $D^{\prime}$ denotes any finitely generated subalgebra of $K$ containing $D$, then $D^{\prime}$, $R_{D^{\prime}}, I_{D^{\prime}}$ also constitute descent data (one must identify $R_{D^{\prime}}$ with a subring of $R$. This is possible because it is free and so torsion-free over $D^{\prime}$, and so, if $L$ denotes the fraction field of $D^{\prime}$, we have $R_{D^{\prime}} \subseteq R_{L} \subseteq R_{K} \cong R$ ). Strictly speaking, we should use the compositum $R_{D}\left[D^{\prime}\right]$ instead of $R_{D^{\prime}}$, but the two are canonically isomorphic, and we identify them.

Note that descent data always exist, for if we represent $R$ as $T / J$, where $T=K\left[x_{1}, \ldots, x_{n}\right]$ is a polynomial ring and $J=\left(f_{1}, \ldots, f_{m}\right) T$, choose $g_{1}, \ldots, g_{r} \in T$ whose images in $R$ generate $I$, and choose $w, u \in T$ whose images in $R$ are $x, c$, respectively, then we may, as a first approximation, take $D$ to be the algebra generated over $Z$ by the coefficients of all of the polynomials $f, g, w, u$, take $R_{D}$ to be $D\left[x_{1}, \ldots, x_{n}\right] /\left(f_{1}, \ldots, f_{m}\right)$, take $I_{D}=\left(g_{\nu}: \nu\right) R_{D}$, and take $x^{\prime}, c^{\prime}$ to be the images of $w, u$, respectively, in $R_{D}$. We then modify $D$ by localizing at one nonzero element (without changing notation) so that $I_{D}, R_{D} / I_{D}$, and hence $R_{D}$ are $D$-free. If $L$ is the fraction field of $D$, it is clear that $R_{D}$ injects into $L \otimes_{D} R_{D}$ and hence into $K \otimes_{L}\left(L \otimes_{D} R_{D}\right) \cong K \otimes_{D} R_{D} \cong R$ by the presentation. Thus, $R_{D}$ may be identified with a subring of $R$. It is easy to see that $x^{\prime}, c^{\prime}$ are identified with $x, c$ once this is done, that $K \otimes_{D} I_{D}=I_{D} R=I$, and that, in fact, all of the conditions which must be satisfied by descent data hold.

We are now ready for

(3.5) Definition. Let $R$ be a finitely generated algebra over a field $K$ of characteristic 0 and $I \subseteq R$. We say that $x \in I^{*}$, the tight closure of $I$, if there exist $c \in R^{\circ}$ and descent data $\left(D, R_{D}, I_{D}\right)$ such that for every maximal ideal $m$ of $D$, if $\kappa=D / m$ and $p$ denotes the characteristic of $\kappa$, then $c_{\kappa} x_{\kappa}^{q} \in I_{\kappa}^{[q]}$ in $R_{\kappa} \cong R_{D} / m R_{D}$ for every $q=p^{e} \gg 0$, where the subscript $\kappa$ denotes images after applying $\kappa \otimes_{D}$. If $I=I^{*}$, we say that $I$ is tightly closed.

It is not even clear from this definition that $I^{*}$ is an ideal. We return to this and other basic characteristic zero issues in [HH7].

We next define tight closure in a slightly larger context. 
(3.6) Definition. Let $R$ be an algebra essentially of finite type over a field $K$ of characteristic 0 , and $I \subseteq R$. We define the tight closure, $I^{*}$, of $I$ as $\bigcup_{B}(I \cap B)^{*}$, where the union is extended over all finitely generated $K$-subalgebras $B$ of $R$ such that $R$ is a localization of $B$. Again, if $I=I^{*}$, we say that $I$ is tightly closed.

(3.7) Remark. We do not know whether the notion given by Definition (3.6) for fields $K$ of characteristic $p$ coincides with the characteristic $p$ definition of tight closure. The difficulty is that we do not know that tight closure commutes with localization.

\section{BASIC PROPERTIES OF TIGHT CLOSURE FOR IDEALS IN CHARACTERISTIC $p$}

The following proposition shows that tight closure has the usual characteristics of a closure operation.

(4.1) Proposition. Let $R$ be a Noetherian ring of characteristic $p$, and let $I$, $J$ be ideals of $R$.

(a) $I^{*}$ is an ideal of $R$ containing $I$.

(b) If $I \subseteq J$, then $I^{*} \subseteq J^{*}$. The intersection of an arbitrary family of tightly closed ideals is tightly closed.

(c) If I has positive height or if $R$ is reduced, then $x \in I^{*}$ iff there exists $c \in R^{\circ}$ such that $c x^{q} \in I^{[q]}$ for all $q=p^{e}$.

(d) For every $I$ there exists $c \in R^{\circ}$ such that $c\left(I^{*}\right)^{[q]} \subseteq I^{[q]}$ for all $q \gg 0$. If $I$ has positive height or if $R$ is reduced, then $c$ can be chosen so that the inclusion holds for all $q$.

(e) $I^{*}=I^{* *}$

(f) $(I \cap J)^{*} \subseteq I^{*} \cap J^{*}$.

(g) $(I+J)^{*}=\left(I^{*}+J^{*}\right)^{*}$.

(h) $(I J)^{*}=\left(I^{*} J^{*}\right)^{*}$.

(i) $(0)^{*}=\operatorname{Rad}(0)$. In particular, $I^{*}$ contains the nilradical of $R$ for all $I \subseteq R$.

(j) For any $I \subseteq R, I^{*}$ is the inverse image in $R$ of $\left(I R_{\mathrm{red}}\right)^{*}$.

(k) If $I$ is tightly closed, then $I: J$ is tightly closed for any ideal $J$.

Proof. (a) and (b) are immediate from the definition. To prove (c), note that an ideal of positive height is generated by the elements in its intersection with $R^{\circ}$, since it is contained in the union of the ideal they generate and the minimal primes. The set of elements satisfying the condition that $c x^{q} \in I^{[q]}$ for all $q$ is an ideal contained in $I^{*}$. It suffices to show that each element of $I^{*} \cap R^{\circ}$ satisfies the stronger condition. Suppose $x \in R^{\circ}$ and $c x^{q} \in I^{[q]}$ for all $q \geq q^{\prime}$. Then we may simply replace $c$ by $c x^{q^{\prime}}$ to see that $x$ satisfies the stronger condition. On the other hand, if $R$ is reduced and $c x^{q} \in I^{[q]}$ for $q \geq q^{\prime}$ with $c \in R^{\circ}$, then since $S=\left(R^{\circ}\right)^{-1} R$ is a finite product of fields, $I S$ is generated by an idempotent and so $I^{[q]} S=I S$ for all $q$. It follows that for each $q<q^{\prime}$, we can choose $c_{q} \in R^{\circ}$ such that $c_{q} x \in I^{[q]}$, and if we then take $c^{\prime}=c\left(\prod_{q<q^{\prime}} c_{q^{\prime}}\right)$, we have $c^{\prime} x^{q} \in I^{[q]}$ for all $q$. 
To prove (d), choose a finite set of generators $f_{i}$ for $I^{*}$, and choose $c_{i} \in R^{\circ}$ for each $i$ such that $c_{i} f_{i}^{q} \in I^{[q]}$ for $q \geq q_{i}$. Then $c=\prod_{i} c_{i}$ has the required property for $q \geq \max _{i}\left\{q_{i}\right\}$. If $I$ has positive height or if $R$ is reduced, choose the $c_{i}$ to work for all $q$.

To prove (e), suppose that $r \in I^{* *}$ and choose $d \in R^{\circ}$ such that $d r^{q} \in\left(I^{*}\right)^{[q]}$ for all $q \geq 0$. Choose $c$ as in part (d). Then $(c d) r^{q} \in I^{[q]}$ for all $q \geq 0$.

(f) is immediate from (b). To prove (g), first note that since $I+J \subseteq I^{*}+J^{*}$, we have $(I+J)^{*} \subseteq\left(I^{*}+J^{*}\right)^{*}$. But, $I^{*}, J^{*} \subseteq(I+J)^{*}$ by (b) and so $I^{*}+J^{*} \subseteq$ $(I+J)^{*}$, and then $\left(I^{*}+J^{*}\right)^{*} \subseteq(I+J)^{* *}=(I+J)^{*}$ by (b) and (e). For h), we have $I J \subseteq I^{*} J^{*}$ and so $(I J)^{*} \subseteq\left(I^{*} J^{*}\right)^{*}$. To prove the other inclusion it suffices to show that $I^{*} J^{*} \subseteq(I J)^{*}$. But if $c u^{q} \in I^{[q]}$ and $d v^{q} \in J^{[q]}$ for all $q \gg 0$, then $c d(u v)^{q} \in I^{[q]} J^{[q]}=(I J)^{[q]}$ for all $q \gg 0$.

For (i), note that $x \in(0)^{*}$ iff for some $c \in R^{\circ}, c x^{q}=0$ for all $q \gg 0$, and this holds iff $c x^{n}=0$ for some $c \in R^{\circ}$ and some $n \in \mathbf{N}$. This forces $x^{n}$ into all minimal primes of $R$, and so $x$ is nilpotent. To prove (j), first observe that the image of $R^{\circ}$ in $R_{\text {red }}$ is $\left(R_{\text {red }}\right)^{\circ}$ and the inverse image of $\left(R_{\text {red }}\right)^{\circ}$ in $R$ is $R^{\circ}$. It is clear that $I^{*}$ maps into $\left(I R_{\text {red }}\right)^{*}$. Now assume that for all $q \geq q^{\prime}$, we have $c x^{q} \in I^{[q]}+N$, where $c \in R^{\circ}$, i.e., $x$ is in the inverse image of $\left(I R_{\text {red }}\right)^{*}$. Choose $q^{\prime \prime}$ such that $N^{\left[q^{\prime \prime}\right]}=0$. Then $c x^{q} \in I^{[q]}$ for all $q \geq q^{\prime} q^{\prime \prime}$, and so $x \in I^{*}$.

To prove (k), first note that since $I: J=\bigcap_{u \in J} I: u$, we may suppose that $J=u R$. Suppose $c \in R^{\circ}$ is such that $c x^{q} \in(I: u)^{[q]}$ for $q \gg 0$. Since $(I: u)^{[q]} \subseteq I^{[q]}: u^{q}, c(x u)^{q} \in I^{[q]}$ for all $q \gg 0$, whence $x u \in I^{*}=I$ and $x \in I: u$.

We note that $*$ does not commute with $\cap$ or : in general. For example, if $R=K\left[\left[x^{2}, x^{3}\right]\right] \subseteq K[[x]]$ and $m=\left(x^{2}, x^{3}\right)$, then $\left(x^{2} R\right)^{*}=m,\left(x^{3} R\right)^{*}=$ $\left(x^{3}, x^{4}\right)$ (by Corollary (5.8), the tight closure of a principal ideal in a domain is the same as its integral closure), and so $\left(x^{2} R\right)^{*} \cap\left(x^{3} R\right)^{*}=\left(x^{3}, x^{4}\right)$, while $x^{2} R \cap x^{3} R=\left(x^{5}, x^{6}\right)$, which is already tightly closed. Likewise, $\left(x^{2} R\right)^{*}$ : $\left(x^{3} R\right)^{*}=m:\left(x^{3}, x^{4}\right)=R$ while $x^{2} R: x^{3} R=m$ is tightly closed.

Neither the sum nor the product of two tightly closed ideals is necessarily tightly closed. In $R=K[[x, y, z]] /\left(x^{2}+y^{8}+z^{8}\right)$, where $K$ is of characteristic $p>2, y R$ and $z R$ are tightly closed, since $R$ is a normal domain, but we shall see in a moment that $y R+z R$ is not. In fact, $x \in(y R+z R)^{*}$. In the same ring, the maximal ideal $m$ is tightly closed (by Theorem (5.2)), but $x \in\left(m^{2}\right)^{*}-m^{2}$. In fact, $x^{2} \in\left(\left(y^{2}, z^{2}\right)^{2}\right)^{2} \Rightarrow x \in\left(\left(y^{2}, z^{2}\right)^{2}\right)^{-} \Rightarrow x \in\left(y^{2}, z^{2}\right)^{*}$ (by Theorem (5.4)), which is contained in both $\left(m^{2}\right)^{*}$ and $(y R+z R)^{*}$. The discussion of Gorenstein rings in [HH5] and the discussion of rational singularities in [HH7] are relevant. 
Our next objective is to prove that in regular rings every ideal is tightly closed. We first note

(4.2) Lemma. Let $R, S$ be arbitrary Noetherian rings such that $S$ is a flat $R$-algebra, and let $I, J$ be ideals of $R$. Then $I S:{ }_{S} J S=\left(I:{ }_{R} J\right) S$, where $I:{ }_{R} J=\{r \in R: r J \subseteq I\}$.

Proof. See [N, Theorem 18.1, part 2].

(4.3) Corollary. In a regular ring $R$ of characteristic $p$, for any two ideals $I$, $J$ we have $I^{[q]}:{ }_{R} J^{[q]}=\left(I:{ }_{R} J\right)^{[q]}$ for all $q$. In particular, $I^{[q]}: x^{q}=(I: x)^{[q]}$ for all $q$.

Proof. The statement is immediate from Lemma (4.2), since the iterated Frobenius endomorphism $F^{e}: R \rightarrow R$ is flat when $R$ is regular (see [Kul, He, PS1]) and $I^{[q]}=F^{e}(I) R$.

The following result, while easy, is extremely important.

(4.4) Theorem. If $R$ is regular, then every ideal is tightly closed.

Proof. Suppose $c \in R^{\circ}$ and $c x^{q} \in I^{[q]}$ for all $q \geq q^{\prime}$ but $x$ is not in $I$. Then these conditions are preserved when we localize at a prime containing $I: x$. Hence, we may assume that $(R, m)$ is regular local and that $I: x \subseteq m$. But then $c \in \bigcap_{q \geq q^{\prime}} I^{[q]}: x^{q}=\bigcap_{q \geq q^{\prime}}(I: x)^{[q]} \subseteq \bigcap_{q \geq q^{\prime}} m^{q}=(0)$, a contradiction.

(4.5) Definition. A Noetherian ring of characteristic $p$ is called weakly $F$ regular if every ideal is tightly closed. If every localization of $R$ at a multiplicative system is weakly F-regular, we say that $R$ is $F$-regular.

With this terminology, Theorem (4.4) immediately yields

(4.6) Theorem. A regular ring of characteristic $p$ is F-regular.

We do not know of an example of a weakly F-regular ring that is not Fregular. To the contrary, what we know suggests that weak F-regularity ought to imply F-regularity, at least for well-behaved rings.

We shall ultimately prove numerous results that either generalize or are parallel to the next theorem. We prefer to give the result in its simplest form early because the proof is transparent and covers many of the most important cases in characteristic $p$.

(4.7) Theorem. Let $R$ be a Noetherian ring of characteristic $p$ module-finite and torsion-free over a regular domain $A$. Let $x_{1}, \ldots, x_{n}$ be elements of $A$ that are parameters in $R$ (cf. Definition (2.1)). Then the colon ideal

$$
\left(x_{1}, \ldots, x_{n-1}\right) R:{ }_{R} x_{n} \subseteq\left(\left(x_{1}, \ldots, x_{n-1}\right) R\right)^{*} .
$$

Proof. We first note that $\left(x_{1}, \ldots, x_{n-1}\right) A:{ }_{A} x_{n}=\left(x_{1}, \ldots, x_{n-1}\right) A$. It suffices to check this locally. Since the equality is automatic if the first ideal in the colon is the unit ideal or if $x_{n}$ is a unit, we need only consider the case where all the $x_{i}$ are in the maximal ideal of $A$. But then they must form an $A$-sequence, for if they were contained in a prime ideal of $A$ of height less than $n$, they would 
also be contained in a prime ideal of $R$ of height less than $n$ lying over it. We therefore need only prove Theorem (4.8) below.

(4.8) Theorem. Let $R$ be a Noetherian ring of characteristic $p$ module-finite and torsion-free over a regular domain $A$. Let $I, J$ be ideals of $A$. Then $I R:{ }_{R} J R \subseteq\left(\left(I:{ }_{A} J\right) R\right)^{*}$ and $I R \cap J R \subseteq((I \cap J) R)^{*}$.

Proof. Let $F \cong A^{t}$ be an $A$-free submodule of $R$ whose rank $t$ is equal to the torsion-free rank of $R$ as an $A$-module. Then $R / F$ is a torsion $A$-module, and we can choose a nonzero element $c \in A$ such that $c R \subseteq F$. Let $x \in I R:{ }_{R} J R$ (resp. $I R \cap J R$ ). Then, for all $q, x^{q} \in I^{[q]} R:{ }_{R} J^{[q]} R$ (resp. $I^{[q]} R \cap J^{[q]} R$ ), whence $c x^{q} \in I^{[q]} F:{ }_{F} J^{[q]}$ (resp. $I^{[q]} F \cap J^{[q]} F$ ). Since $F$ is $A$-free, we see $c x^{q} \in\left(I^{[q]}:{ }_{A} J^{[q]}\right) F$ (resp. $\left(I^{[q]} \cap J^{[q]}\right) F$ ), and by the flatness of the Frobenius endomorphism of $A$, we then have that $c x^{q} \in\left(I:{ }_{A} J\right)^{[q]} F \subseteq\left(\left(I:{ }_{A} J\right) R\right)^{[q]}$ (resp. $\left.(I \cap J)^{[q]} F \subseteq((I \cap J) R)^{[q]}\right)$ for all $q$, which yields the desired result.

(

We note that the argument given is quite similar to the use of amiable systems of parameters in [Ho3].

(4.9) Theorem. Let $R$ be a Noetherian ring of characteristic $p$ module-finite and torsion-free over a regular domain $A$. If every ideal generated by parameters is tightly closed, then $R$ is $C$-M. In particular, if $R$ is F-regular or even weakly $F$-regular, then $R$ is Cohen-Macaulay.

Proof. Let $P$ be a prime ideal of $R$ of height $n$. Then we can choose $x_{1}, \ldots$, $x_{n} \in P \cap A$ such that $\left(x_{1}, \ldots, x_{i}\right) A$ has height $i$ for $0 \leq i \leq n$, since the height of $P \cap A$ is also $n$, and it follows that $x_{1}, \ldots, x_{i}$ are parameters in $R$ for every $i$. By Theorem (4.7), we have that $\left(x_{1}, \ldots, x_{i-1}\right) R:{ }_{R} x_{i} \subseteq$ $\left(x_{1}, \ldots, x_{i-1}\right) R^{*}=\left(x_{1}, \ldots, x_{i-1}\right) R$ for every $i$, and so $x_{1}, \ldots, x_{n}$ is a regular sequence in $R$, and $\operatorname{depth}_{P} R=n$. Since this holds for every prime ideal of $R, R$ is C-M.

Later, we shall relax the condition that $R$ be module-finite over a regular ring in various ways. But we want to point out that the present result suffices to prove

(4.10) Theorem. Let $R \subseteq S$, where $S$ is regular of characteristic $p$, and suppose that $R$ is a direct summand of $S$ as an $R$-module or, more generally, that $R$ is a pure subring of $S$ (cf. §2). Then $R$ is $C-M$.

Proof. It is easy to reduce to the case where $R$ is complete local and $S$ is a regular domain. See the beginning of $\S 7$ of [HR1, pp. 141-142]. In this situation, every ideal of $R$ is contracted from $S$. The result follows at once from Proposition (4.12) below and Theorem (4.9), since the complete local ring $R$ is a domain and module-finite over a regular ring.

(4.11) Lemma. Let $R, S$ be Noetherian rings of characteristic $p$. Suppose $h$ is a homomorphism from $R$ to $S$. If $h\left(R^{\circ}\right) \subseteq S^{\circ}$ (which is equivalent to the 
assertion that every minimal prime of $S$ contracts to a minimal prime of $R$ ), then we have the following.

(a) If $I \subseteq R$, then $h\left(I^{*}\right) \subseteq(I S)^{*}$.

(b) If $J$ is tightly closed in $S$, its contraction to $R$ is tightly closed in $R$.

In particular, (a), (b) hold if $S$ is a domain containing $R$, if $S$ is a localization of $R$, and, more generally, if the going-down theorem holds for $R \rightarrow S$.

Proof. To see (a), note that $c \in R^{\circ}$ and $c x^{q} \in I^{[q]}$ for all $q \gg 0$ implies that $h(c) \in S^{\circ}$ and $h(c) h(x)^{q} \in(I S)^{[q]}$ for all $q \gg 0$. (b) is immediate from (a).

(4.12) Proposition. Let $R \subseteq S$ be Noetherian rings of characteristic $p$ such that every ideal of $R$ is contracted from $S$ (which holds, in particular, when $R$ is a direct summand of $S$ as an $R$-module or when $R$ is pure in $S$ ) and $R^{\circ} \subseteq S^{\circ}$. If $S$ is $F$-regular or weakly $F$-regular, then $R$ has the same property.

Proof. Since the hypothesis is stable under localization (cf. [Ho5], where the contractedness condition is called "cyclic purity"), this follows immediately from Lemma (4.11).

The characteristic zero version of this result will be given in [HH7]. It is important to note that when a linearly reductive linear algebraic group over a field $K$ acts $K$-rationally on a $K$-algebra $R$, the ring of invariants $R^{G}$ is a direct summand of $R$ as an $R^{G}$-module. If $R$ is regular, F-regular, or weakly Fregular, it will follow that $R^{G}$ has the same property and so is Cohen-Macaulay. A result of this type was first obtained in [HR1], by reduction to characteristic $p$. Other proofs were given in $[\mathrm{Ke}]$ and $[\mathrm{B}]$. The results of $[\mathrm{B}]$ suggest a connection between F-regularity and rational singularity. This is pursued in [HH7]. See also [Bor, HoE, Hol, and HR2] for further background.

(4.13) Remark. Let $R$ be a product $\prod_{i=1}^{k} R_{i}$. We note that $R^{\circ}=\prod_{i=1}^{k} R_{i}^{\circ}$. Every ideal $I$ of $R$ has the form $\prod_{i=1}^{k} I_{i}$, where $I_{i} \subseteq R_{i}$. We also note that $I^{*}=\prod_{i=1}^{k} I_{i}^{*}$. The verification is straightforward and is left to the reader. It follows that $R$ is (weakly) F-regular iff each $R_{i}$ is.

We do not know, in general, how tight closure behaves under localization. However, the situation with respect to localization at a maximal ideal could not be better.

(4.14) Proposition. Let $R$ be a Noetherian ring of characteristic $p$, and let $I$ be an ideal primary to a maximal ideal $m$. Then $\left(I R_{m}\right)^{*}=I^{*}\left(R_{m}\right)$. Hence, if $I$ is tightly closed, so is $I R_{m}$. Moreover, $I^{*}$ is the contraction of $\left(I R_{m}\right)^{*}$.

Proof. It is clear that $R^{\circ}$ maps into $\left(R_{m}\right)^{\circ}$, whence $I^{*}$ maps into $\left(I R_{m}\right)^{*}$ and $\supseteq$ follows. Now let $x$ be an element of $R$ such that $x / 1 \in\left(I R_{m}\right)^{*}$. We must show that $x \in I^{*}$. We can choose $c \in R$ such that $c / 1 \in\left(R_{m}\right)^{\circ}$ and $(c / 1)(x / 1)^{q} \in I^{[q]} R_{m}$ for all $q \gg 0$. We first claim that we can choose $c \in R^{\circ}$. To see this, let $\delta$ be an element of $R$ that belongs precisely to those minimal primes of $R$ to which $c$ does not belong. Then $\delta$ is in every minimal prime 
contained in $m$, and so is nilpotent in $R_{m}$. Replacing $\delta$ by a power, we may assume $\delta / 1$ is 0 in $R_{m}$. We may then replace $c$ by $c+\delta$, which is clearly in $R^{\circ}$. Since $I$ is $m$-primary, so is each $I^{[q]}$. This uses strongly that $m$ is maximal. Since $c x^{q} / 1 \in I^{[q]} R_{m}$ for all $q \gg 0, c x^{q} \in I^{[q]}$ for all $q \gg 0$.

(4.15) Corollary. $R$ is weakly $F$-regular iff $R_{m}$ is weakly $F$-regular for every maximal ideal $m$.

This is immediate from Propositions (4.14) and (4.16) below. Proposition (4.16) is clear from the fact that every ideal in a Noetherian ring is an intersection of ideals primary to maximal ideals.

(4.16) Proposition. A Noetherian ring of characteristic $p$ is weakly F-regular if and only if every ideal primary to a maximal ideal is tightly closed.

We conclude this section with a discussion of the obstruction to proving that tight closure commutes with localization. In fact, we consider the easier problem of proving that a localization of a weakly F-regular ring at an arbitrary prime (and, hence, at an arbitrary multiplicative system) is weakly F-regular. Since we can localize at maximal ideals and then move down to an arbitrary prime by localizing repeatedly at primes of coheight one, we reduce at once to considering the following situation. $(R, m)$ is local and weakly F-regular, $P$ is a prime ideal of $R$ such that $\operatorname{dim} R / P=1$, and we want to show that every ideal of $R_{P}$ is tightly closed. It suffices to show that for each ideal $I$ of $R$ primary to $P, I$ remains tightly closed upon localization at $P$. Let $f$ be an element of $R$ whose image in $R / P$ is a parameter. It suffices, then, to show that a $P$-primary ideal $I$ remains tightly closed when expanded to $R_{f}$, for $P R_{f}$ is a maximal ideal of $R_{f}$ and so localizing at $P$ does not present a problem once we have localized at $f$.

Suppose that $u$ is an element of $R$ that is in $\left(I R_{f}\right)^{*}$. We want to show that $u \in I^{*}$. We know that for a certain $c \in R^{\circ}$ and for all $q, c u^{q} \in I^{[q]} R_{f}$, and so we know that for every $q$ there is a positive integer $N(q)$ depending on $q$ such that $c f^{N(q)} u^{q} \in I^{[q]}$. for all $q$.

Approached this way, the problem is that we cannot prove a sufficiently good bound for the integer $N(q)$. It would suffice if we could show that there is a constant integer $B>0$ such that $N(q)$ could be chosen $\leq B q$ for all $q$. For then $c\left(f^{B} u\right)^{q} \in I^{[q]}$ for all $q$, and this yields $f^{B} u \in I^{*}=I$ and then $u \in I$, since it is $P$-primary.

The fact that $c u^{q}$ is killed by a power of $f$ modulo $I^{[q]}$ means that it represents an element of $H_{f R}^{\circ}\left(R / I^{[q]}\right)=H_{m}^{\circ}\left(R / I^{[q]}\right)$. The limited problem of localization that we are considering would be solved if we could show that there is a fixed integer $B>0$ such that $f^{B q}$ kills $H_{m}^{\circ}\left(R / I^{[q]}\right)$ for all $q$. However, we have not been able to prove such a bound even for very good rings $R$ (not even for complete, normal, Cohen-Macaulay domains, and not even if $R$ is weakly F-regular). On the other hand, it appears to be possible that such bounds exist even under much weaker hypotheses.

When $R$ is reduced and every ideal of $R$ is contracted from $R^{1 / p}$ (and, hence, from $R^{\infty}$ as well), it would suffice to prove instead that there is an 
integer $B>0$ such that $f^{B}$ kills $H_{m}^{\circ}\left(R^{\infty} / I R^{\infty}\right)$. This is equivalent to the assertion that the annihilators of the powers of $f$ in $R^{\infty} / I R^{\infty}$ stabilize. This version of the problem does not appear to be any more tractable than the other.

Of course, one may ask whether localization commutes with tight closure in even more general situations. Attacking this question in general leads to similar problems on bounding exponents that, naturally, are even more difficult.

\section{INTEGRAL CLOSURE AND THE BRIANÇON-SKODA THEOREM IN CHARACTERISTIC $p$}

(5.1) Remarks on integral closure of ideals. In the next four paragraphs we suspend the conditions that rings be Noetherian and that the characteristic be $p$ and discuss integral closure of ideals in arbitrary commutative rings with identity. We recall that an element $x$ of a ring $R$ is integral over an ideal $I$ provided there exists a positive integer $k$ and an equation

$$
x^{k}+i_{1} x^{k-1}+\cdots+i_{j} x^{j}+\cdots+i_{k-1} x+i_{k}=0,
$$

where $i_{j} \in I^{j}$ for $1 \leq j \leq k$. This is easily seen to be equivalent to the assertion that there is an integer $k \geq 1$ such that $x^{k} \in I(I+R x)^{k-1}$, and this holds iff $(I+R x)^{k}=I(I+R x)^{k-1}$. From this last equation it is trivial to prove by induction on $m$ that

$$
(I+R x)^{k+m}=I^{m+1}(I+R x)^{k-1}
$$

for every integer $m \in \mathbf{N}$. Thus, $x$ is integral over $I$ iff there exists a positive integer $k>0$ such that (\#) holds for all $m \in \mathbf{N}$.

We also note that the set of elements $I^{-}$integral over $I$ is an ideal, called the integral closure of $I$. The integral closure of $I$ may be characterized alternatively as follows. If $t$ is an indeterminate over $R$, the integral closure of the Rees ring $R[I t]$ in the polynomial ring $R[t]$ is

$$
\sum_{j=0}^{\infty}\left(I^{j}\right)^{-} t^{j}=R+I^{-} t+\left(I^{2}\right)^{-} t^{2}+\cdots+\left(I^{j}\right)^{-} t^{j}+\cdots,
$$

and when $R$ is a normal domain, we may even characterize the displayed ring as the integral closure of $R[I t]$ in its fraction field.

Yet another characterization of integral closure for ideals is given by valuations. Let $R$ be a ring with finitely many minimal prime ideals (this is, of course, automatic when $R$ is Noetherian) and $I \subseteq R$. Then $x$ is integral over $I$ iff for every homomorphism $h$ of $R$ into a valuation domain $V$ such that Ker $h$ is a minimal prime of $R, h(x) \in I V$. If $R$ is Noetherian, the same result holds with $V$ restricted to being a discrete valuation ring (by which we always mean a rank one discrete valuation ring).

Finally, we remark that if $R \subseteq S$ is an integral extension of rings and $I \subseteq R$ is an ideal, then $I S \cap R \subseteq I^{-}$.

We refer the reader to [L] for background on integral closures of ideals.

This completes our general discussion of integral closure of ideals. We now return to our usual notational conventions. Moreover, throughout the remainder of this section all rings will have characteristic $p$. 
(5.2) Theorem. Let $R$ be a Noetherian ring of characteristic $p$ and $I \subseteq R$ an ideal. Then $I^{*} \subseteq I^{-}$. In particular, every integrally closed ideal, and, hence, every radical ideal is tightly closed.

Proof. Suppose $x \in I^{*}$ and $c \in R^{\circ}$ is such that $c u^{q} \in I^{[q]}$ for all $q$. Let $h: R \rightarrow V$ have as its kernel a minimal prime of $R$, where $V$ is a DVR. Then $h(c) h(u)^{q} \in(I V)^{[q]}$ for all $q$ and $h(c) \neq 0$. Thus, $h(u) \in(I V)^{*}=I V$ (since $V$ is regular).

Alternate proof. Let $I=\left(x_{1}, \ldots, x_{h}\right)$. Applying the discrete valuation $v$ to the equation $c x^{q}=\sum_{t=1}^{h} r_{q t} x_{t}^{q}$ yields $v(c)+q v(x) \geq q \min \left\{v\left(x_{t}\right): t\right\}$. Dividing by $q$ and taking the limit as $q \rightarrow \infty$ yields the result.

(5.3) Example. If $x$ and $y$ are any two elements of a ring $R$, then $\left(x^{n}, y^{n}\right)^{-}$ $\supseteq\left(x^{n}, x^{n-1} y, \ldots, x^{n-i} y^{i}, \ldots, x y^{n-1}, y^{n}\right)=(x, y)^{n}$, since the monomial $x^{n-i} y^{i}$ satisfies $z^{n}-\left(x^{n}\right)^{n-i}\left(y^{n}\right)^{i}=0$. On the other hand, if $R$ is regular or F-regular, e.g., if $R=K[x, y]$, where $K$ is a field, then $\left(x^{n}, y^{n}\right)^{*}=\left(x^{n}, y^{n}\right)$, since every ideal is tightly closed. Thus, the tight closure is, in general, much smaller than the integral closure. The tight closure is a "tight fit" for the original ideal, which is the reason for the choice of the term.

We are now in a position to prove a result, at least in characteristic $p$, which greatly generalizes the Briançon-Skoda theorem (cf. [BrS, LT, and LS]).

(5.4) Theorem (generalized Briançon-Skoda theorem). Let $R$ be a Noetherian ring of characteristic $p$, and let $I$ be an ideal of positive height generated by $n$ elements, say $u_{1}, \ldots, u_{n}$. Then for every $m \in \mathbf{N},\left(I^{n+m}\right)^{-} \subseteq\left(I^{m+1}\right)^{*}$. In particular, $\left(I^{n}\right)^{-} \subseteq I^{*}$.

Hence, if $R$ is weakly F-regular and, in particular, if $R$ is regular, then $\left(I^{n+m}\right)^{-} \subseteq I^{m+1}$ and $\left(I^{n}\right)^{-} \subseteq I$.

Proof. If $\left(I^{n+m}\right)^{-}$is contained in the union of $\left(I^{m+1}\right)^{*}$ and the minimal primes of $R$, then it must be contained in one of them, and then it must be contained in $\left(I^{m+1}\right)^{*}$, since height $I$ is positive. Hence, we assume to the contrary that it is not contained in this union, and we choose $y \in\left(I^{n+m}\right)^{-}-\left(I^{m+1}\right)^{*}$ not in any minimal prime of $R$. Let $J=I^{n+m}$. By Remark (5.1) we can choose an integer $k>0$ such that $(J+y R)^{k+h}=J^{h+1}(J+y R)^{k-1}$ for every $h \in \mathbf{N}$, and then $\left(y^{k}\right) y^{h} \in J^{h}$ for all $h \in \mathbf{N} . J^{h}=I^{h n+h m}$ is generated by monomials of degree $h n+h m$ in the $u_{i}$. Then $I^{h n+h m} \subseteq\left(u_{1}^{h}, \ldots, u_{n}^{h}\right)^{m+1}$. (We may assume $h>0$. Consider a monomial generator $v$ of $I^{h n+h m}$ in which the exponent of $u_{i}$ is $b_{i}$, $1 \leq i \leq n$, where $\sum_{i} b_{i}=h n+h m$. Let $a_{i}$ be the integer part of $b_{i} / h$ for each $i$. Then $a_{i}+1>b_{i} / h$, and so $\sum_{i}\left(a_{i}+1\right)=\left(\sum_{i} a_{i}\right)+n>\left(\sum_{i} b_{i}\right) / h=n+m$, so that $\sum_{i} a_{i}>m$, i.e., $\sum_{i} a_{i} \geq m+1$, whence

$$
\prod_{i}\left(u_{i}^{h}\right)^{a_{i}} \in\left(u_{1}^{h}, \ldots, u_{n}^{h}\right)^{m+1}
$$

and divides the given monomial generator $v$ of $I^{h n+h m}$.) Then we may take $c=y^{k} \in R^{\circ}$, and when $h$ has the form $q=p^{e}$, we obtain $c y^{q} \in J^{q} \subseteq$ $\left(u_{1}^{q}, \ldots, u_{n}^{q}\right)^{m+1}=\left(I^{m+1}\right)^{[q]}$, and so $y \in\left(I^{m+1}\right)^{*}$. This completes the proof of 
the first statement. The second is simply the case $m=0$. The final statement is immediate from the definition of a weakly F-regular ring.

(5.5) Remarks on analytic spread. If $J \subseteq I=J^{-}$, where $J$ has $n$ generators and height $I>0$, then $\left(I^{m+n}\right)^{-} \subseteq\left(I^{m+1}\right)^{*}$ in this case as well, since $\left(I^{m+n}\right)^{-}=$ $\left(J^{m+n}\right)^{-} \subseteq\left(J^{m+1}\right)^{*} \subseteq\left(I^{m+1}\right)^{*}$. If $R$ is local with infinite residue class field, then every ideal is integral over an ideal generated by at most $\operatorname{dim} R$ elements. See [NoR1]. Hence, in this situation, we may take $n$ to be the minimum of the number of generators of $I$ and the dimension of the ring in the statement of Theorem (5.4). More generally, we may take $n$ to be the analytic spread $a(I)$ of $I$ (the Krull dimension of $K \otimes_{R} \mathrm{gr}_{I} R$ ) when $R$ is local with residue field $K$, since $I$ is integral over an ideal generated by $a(I)$ elements when $K$ is infinite. However, the assumption that $K$ be infinite is not essential. If $K=R / m$ is finite, choose $y$ in $R$ as in the proof of Theorem (5.4) and then carry through the argument in $R(t)$ (the localization of $R[t]$ at $m R[T]$ ) working with an $a(I)$-generated ideal $I^{\prime} \subseteq R(t)$ over which $I$ is integral. One then obtains $c y^{q} \in\left(I^{\prime m+1}\right)^{[q]} \subseteq\left(I^{m+1}\right)^{[q]} R(t)$. Then, since $R(t)$ is faithfully flat over $R$, we have $c y^{q} \in\left(I^{m+1} R\right)^{[q]}$, and the result follows. (Note that $c$ is a fixed power of $y$ and hence in $R$.) Formally

(5.6) Theorem. Let $R$ be any Noetherian local ring of characteristic $p$. Let $I$ be an ideal of positive height. Then $\left(I^{m+a(I)}\right)^{-} \subseteq\left(I^{m+1}\right)^{*}$ for all $m \in \mathbf{N}$. In particular, $\left(I^{a(I)}\right)^{-} \subseteq I^{*}$

(5.7) Corollary. Let $R$ be a weakly F-regular ring of characteristic $p$, and let $I$ be an ideal of positive height. Let $a(I)=\sup a\left(I R_{m}\right)$ for maximal ideals $m$ containing $I$. Then $\left(I^{m+a(I)}\right)^{-} \subseteq I^{m+1}$ for all $m \in \mathbf{N}$. In particular, $\left(I^{a(I)}\right)^{-} \subseteq I$.

Proof. If not, we can obtain a counterexample after localizing at a maximal ideal $m$. But $R_{m}$ is still weakly F-regular, by Corollary (4.15), and we may now apply Theorem (5.6).

(5.8) Corollary. Let $R$ be a Noetherian ring of characteristic $p$, and let $x$ be an element of $R^{\circ}$. Then $(x)^{*}=(x)^{-}$.

Proof. $(x)^{*} \subseteq(x)^{-}$by Theorem (5.2). The other inclusion is immediate from Theorem (5.4) by taking $n=1, m=0$.

(5.9) Lemma. Let $R$ be an arbitrary Noetherian ring such that no prime is both minimal and maximal, i.e., such that $\operatorname{Spec}(R)$ has no zero-dimensional component. Suppose that every principal ideal of $R$ of height one is integrally closed. Then $R$ is normal.

Proof. Let $N$ denote the nilradical of $R$. Every integrally closed ideal contains $N$. For each minimal prime $P$ of $R$, we may choose an element not in any minimal prime and not invertible modulo $P$ (if $Q$ is any prime containing $P, Q$ is not contained in the union of the minimal primes). The product $a$ of these elements is in $R^{\circ}$ and not invertible modulo any minimal prime. 
$N \subseteq \bigcap_{i}\left(a^{i} R\right)^{-}=\bigcap_{i}\left(a^{i} R\right)$, whence there exists an element $r \in R$ such that $b N=0$ with $b=1-r a$. But then $b \in R^{\circ}$, and there exists an element $r^{\prime} \in R$ such that $\left(1-r^{\prime} b\right) N=0$, by the same argument, and so $N=0$.

Thus, $R$ is reduced. Let $r / s, r, s \in R, s$ a nonzerodivisor, be in the total quotient ring of $R$ and be integral over $R$. Then $r$ is integral over $s R$, and so $r / s \in R$.

(5.10) Corollary. Let $R$ be a Noetherian ring of characteristic $p$ such that no prime is both minimal and maximal. If every principal ideal of height one is tightly closed, then $R$ is normal.

\section{(5.11) Corollary. $A$ weakly F-regular ring $R$ is normal.}

Proof. Since (0) is tightly closed, $R$ is reduced. If $R$ is a product with some 0-dimensional factors, they must be fields, while the other factors are weakly F-regular by Remark (4.13) and hence normal by Corollary (5.10).

\section{TEST ELEMENTS}

In the definition of tight closure, the multiplier $c$ is permitted to vary in $R^{\circ}$ as $I$ changes when one tests whether various elements are in $I^{*}$. For many important choices of $R$, it is not necessary to let $c$ vary. A single choice of $c$ can be used for all tight closure tests. We explore this phenomenon in this section.

If $R$ is a Noetherian ring of characteristic $p$, we shall say that $c \in R^{\circ}$ is a $q^{\prime}$-weak test element if there exists $q^{\prime}$ such that for all $I \subseteq R$ and all $x \in I^{*}$, we have $c x^{q} \in I^{[q]}$ for all $q \geq q^{\prime}$. If this holds with $q^{\prime}=1$, we call $c$ a test element. Note that if $J$ is the ideal of $R$ generated by the (weak) test elements, then every element of $J \cap R^{\circ}$ is a (weak) test element. It is not clear, in general, whether $R$ has a test element. Note, for example, that if $R$ is weakly F-regular, then 1 (and, for that matter, every element of $R^{\circ}$ ) is a test element.

We say that an element of $R$ is a locally stable $q^{\prime}$-weak test element if its image in every local ring of $R$ is also a $q^{\prime}$-weak test element. Finally, we say that an element of $R$ is a completely stable $q^{\prime}$-weak test element if it is locally stable and its image in the completion of each local ring of $R$ is a $q^{\prime}$-weak test element. When $q^{\prime}=1$, we omit the phrase " $q^{\prime}$-weak."

We shall see, in fact, that a reduced ring $R$ that is a localization of a torsionfree module-finite extension of a regular domain $A$ has a completely stable $q^{\prime}$-weak test element and that if $R$ is, in addition, generically smooth over $A$, it has a completely stable test element. If $R$ is reduced, equidimensional, and either essentially of finite type over a field or a localization of a complete local ring, then, likewise, $R$ has a completely stable test element. Moreover, we shall also see that the existence of test elements provides a technique for transition between a local ring and its completion in studying questions about tight closure.

In $\S 8$, we shall show that the theory of test elements that we develop for ideals in this section extends without essential modification to the case of tight closure of submodules of a module. In all cases where we have been able to construct test elements, they work for modules as well as ideals. However, we do not know whether this is true in general. A test element for ideals is automatically a 
test element for modules if the ring is excellent and reduced (more generally, if its local rings are approximately Gorenstein; see (8.6) and Proposition (8.15)), but we do not know the corresponding fact for weak test elements. From $\S 8$ on, we change terminology and use the term "(weak) test element" to mean (weak) test element for modules. If we need to refer to the notion introduced in this section, we use the term "(weak) test element for ideals."

In [HH5] (see also [HH3]), we use a different circle of ideas, connected with the notion of strong F-regularity, to construct test elements (for ideals and modules) in the situation where $R$ has characteristic $p$, is reduced, and $R^{1 / p}$ is module-finite over $R$. We show there that, under the hypotheses just specified, if $c \in R^{\circ}$ and $R_{c}$ is regular (or, more generally, strongly F-regular, see [HH3]), then $c$ has a power that is a completely stable test element. This circle of ideas eventually yields a convoluted argument which shows that if $R$ is reduced and finitely generated over an excellent local ring of characteristic $p$, then every element $c \in R^{\circ}$ such that $R_{c}$ is regular (or F-regular Gorenstein) has a power which is a completely stable test element.

The following result establishes both the usefulness and some of the basic properties of test elements.

(6.1) Proposition. Let $R$ be Noetherian of characteristic $p$ and $c \in R$.

(a) $c$ is a $q^{\prime}$-weak test element for $R$ iff $c / 1$ is a $q^{\prime}$-weak test element for $R_{m}$ for every maximal ideal $m$ of $R$.

(b) If $R$ has a weak test element, then the tight closure of $I \subseteq R$ is the intersection of the tightly closed ideals containing $I$ that are primary to a maximal ideal.

(c) If $c \in R$ is a $q^{\prime}$-weak test element for the completion $\widehat{R}$ of the local ring $R$, then it is also a $q^{\prime}$-weak test element for $R$. In this case, for all $I \subseteq R$ and $x \in R, x \in I^{*}$ if and only if $x \in(I \widehat{R})^{*}$, i.e., $I^{*}=(I \widehat{R})^{*} \cap R$.

(d) Let $N$ be the ideal of nilpotents in $R$ and suppose that $N^{\left[q^{\prime \prime}\right]}=0$. Let $c^{\prime}$ be the image of $c \in R$ in $R_{\mathrm{red}}$. If $c$ is a $q^{\prime}$-weak test element for $R$, then $c^{\prime}$ is a $q^{\prime}$-weak test element in $R_{\mathrm{red}}$. If $c^{\prime}$ is a $q^{\prime}$-weak test element in $R_{\mathrm{red}}$, then $c^{q^{\prime \prime}}$ is a $q^{\prime} q^{\prime \prime}$-weak test element in $R$.

Proof. (a) Suppose that $c$ is a $q^{\prime}$-weak test element for $R$. Suppose that $x / 1 \in\left(I R_{m}\right)^{*}$ and $q \geq q^{\prime}$. We must show that $c x^{q} / 1 \in I^{[q]} R_{m}$. If not, we can choose a positive integer $t$ such that $c x^{q} / 1 \notin m^{t} R_{m}+I^{[q]} R_{m}$ and then $c x^{q} / 1 \notin\left(m^{t}+I\right)^{[q]} R_{m}$, which implies that $c x^{q} \notin\left(m^{t}+I\right)^{[q]}$ and hence that $x \notin\left(m^{t}+I\right)^{*}$, since $c$ is a test element in $R$. But then Proposition (4.14) implies that $x / 1 \notin\left(\left(m^{t}+I\right) R_{m}\right)^{*}$ and so $x / 1 \notin\left(I R_{m}\right)^{*}$, a contradiction.

Conversely, suppose that $c / 1$ is a $q^{\prime}$-weak test element for $R_{m}$ for every maximal ideal $m$ of $R$. This implies that $c \in R^{\circ}$. Suppose that $I \subseteq R$, $x \in I^{*}$, and $q \geq q^{\prime}$, but $c x^{q} \notin I^{[q]}$. Then we can localize at a maximal ideal $m$ of $R$ so as to preserve the last condition, and we still have $x / 1 \in\left(I R_{m}\right)^{*}$, which contradicts the condition that $c / 1$ be a $q^{\prime}$-weak test element for $R_{m}$. 
(b) One inclusion is obvious. To prove the other, suppose that $x \in R-I^{*}$. We must construct an ideal $J \supseteq I$ primary to a maximal ideal such that $x \notin$ $J^{*}$. Since $x \notin I^{*}$, we can choose $q \geq q^{\prime}$ such that $c x^{q} \notin I^{[q]}$. We can choose a maximal ideal $m$ such that $c x^{q} / 1 \notin I^{[q]} R_{m}$ and then $t$ such that $c x^{q} / 1 \notin m^{t} R_{m}+I^{[q]} R_{m}$. We then conclude exactly as in the proof of part (a) that $x \notin\left(m^{t}+I\right)^{*}$.

(c) Since $\widehat{R}$ is $R$-flat, $R^{\circ} \subseteq \widehat{R}^{\circ}$, and so $I^{*} \subseteq(I \widehat{R})^{*}$ for $I \subseteq R$. Let $c \in R$ be a $q^{\prime}$-weak test element for $\widehat{R}$. The results claimed all follow if we can prove that for all $I \subseteq R$ and $x \in R$, if $x \in(I \widehat{R})^{*}$, then $c x^{q} \in I^{[q]}$ for all $q \geq q^{\prime}$. But since $c$ is a $q^{\prime}$-weak test element for $\widehat{R}$, we have $c x^{q} \in(I \widehat{R})^{[q]}=I^{[q]} \widehat{R}$, and since $\widehat{R}$ is faithfully flat over $R$, this implies that $c x^{q} \in I^{[q]}$, as required.

(d) We have that $c \in R^{\circ}$ iff $c^{\prime} \in\left(R_{\text {red }}\right)^{\circ}$. Suppose that $c$ is a $q^{\prime}$-weak test element in $R$. If $I \supseteq N$ is an ideal of $R$ such that $u+N \in(I / N)^{*}$, then $u \in I^{*}$ by Proposition (4.1)(j), and so $c u^{q} \in I^{[q]}$ for all $q \geq q^{\prime}$, which implies that $c^{\prime}(u+N)^{q} \in(I / N)^{[q]}$ for all $q \geq q^{\prime}$. On the other hand, if $c^{\prime}$ is a $q^{\prime}$-weak test element in $R_{\text {red }}$ and $I$ is any ideal of $R$, then $u \in I^{*}$ implies $u+N \in(I+N / N)^{*}$, which implies in turn that $c^{\prime}(u+N)^{q} \in(I+N / N)^{[q]}$, and so $c u^{q} \in I^{[q]}+N$ for $q \geq q^{\prime}$. Raising to the $q^{\prime \prime}$ power yields $c^{q^{\prime \prime}} u^{q} \in I^{[q]}$ for all $q \geq q^{\prime} q^{\prime \prime}$.

(6.2) Corollary. (a) Let $c$ be a locally stable $q^{\prime}$-weak test element for $R$. Then $c / 1$ is a $q^{\prime}$-weak test element for every localization $U^{-1} R$ of $R$. In particular, $c$ is a $q^{\prime}$-weak test element for $R$.

(b) In order that $c$ be a completely stable $q$-weak test element for $R$, it suffices that its image be a test element in the completion of each local ring of $R$.

(c) Let $R, N, q^{\prime \prime}, c$, and $c^{\prime}$ be as in Proposition (6.1)(d). If $c$ is a locally stable $q^{\prime}$-weak test element for $R$, then $c^{\prime}$ is a locally stable $q^{\prime}$-weak test element in $R_{\mathrm{red}}$. If $c^{\prime}$ is a locally stable $q^{\prime}$-weak test element in $R_{\mathrm{red}}$, then $c^{q^{\prime \prime}}$ is a locally stable $q^{\prime} q^{\prime \prime}$-weak test element in $R$.

(d) Let $R, N, q^{\prime \prime}, c$, and $c^{\prime}$ be as in Proposition (6.1)(d), and assume, moreover, that $R$ has reduced formal fibers. If $c$ is a completely stable $q^{\prime}$-weak test element for $R$, then $c^{\prime}$ is a completely stable $q^{\prime}$-weak test element in $R_{\text {red }}$. If $c^{\prime}$ is a completely stable $q^{\prime}$-weak test element in $R_{\text {red }}$, then $c^{q^{\prime \prime}}$ is a completely stable $q^{\prime} q^{\prime \prime}$-weak test element in $R$.

Proof. (a), (b), and (c) are immediate from Proposition (6.1) (note in (c) that we can use the same $q^{\prime \prime}$ to kill the nilradical in every localization). For part (d), we need only remark that the hypothesis on the formal fibers implies that the nilradical of the completion of a local ring of $R$ is generated by the image of $N$, so that again we can use the same $q^{\prime \prime}$.

The rest of this section is primarily devoted to establishing the existence of completely stable $q^{\prime}$-weak test elements for certain module-finite extensions of regular rings. Because oi our results on passage to $R_{\text {red }}$, our focus is on the 
reduced case. We begin by treating the generically smooth case. In the latter parts of this section, we explain how to extend the results for this situation to greater generality.

(6.3) Discussion. Let $A$ be a regular domain of characteristic $p$, and let $R$ be module-finite, torsion-free, and generically smooth over $A$. The last condition means that $L \otimes_{A} R$ is smooth over the fraction field $L$ of $A$, which in turn means that $L \otimes_{A} R$ is a finite product of fields each of which is a finite, separable extension of $L$. This implies that we can find $d \in A^{\circ}$ such that $R_{d}$ is smooth over $A_{d}$. Note that $R \subseteq R_{d}$, which is regular and, hence, reduced. When $S$ is smooth and module-finite over the reduced ring $B$ (which implies $S$ is reduced as well), we have, quite generally, that $S^{1 / q} \cong B^{1 / q} \otimes_{B} S$, for all $q=p^{e}$, where the map from the second ring to the first is induced by the obvious $B$-algebra maps from $B^{1 / q}$ and $S$ into $S^{1 / q}$. (Both sides are module-finite over $B^{1 / q}$, and the issue is local on $B^{1 / q}$ and so on $B$. Both sides are $B^{1 / q}$-flat and so free in the local case, and by Nakayama's lemma over $B^{1 / q}$, it suffices to see that we have an isomorphism after killing the maximal ideal of $B^{1 / q}$. In this way, we reduce to the case where the local ring $B$ is replaced by its residue field and $S$ is a separable extension of a field.) Letting $B=A_{d}$ and $S=R_{d}$, we see that with $d$ as above we have $\left(R_{d}\right)^{1 / q} \cong\left(A_{d}\right)^{1 / q} \otimes R_{d}$ (over $A_{d}$ ) from which we deduce

(6.4) Lemma. Let $R$ be a ring of characteristic $p$ module-finite, torsion-free, and generically smooth over a regular ring $A$. Let $d$ be an element of $A^{\circ}$ such that $R_{d}$ is $A_{d}$-smooth. Then $d$ has a power $b$ such that $b R^{1 / p} \subseteq A^{1 / p}[R] \cong$ $A^{1 / p} \otimes_{A} R$. Let $c=b^{2}$. Then $c R^{1 / q} \subseteq A^{1 / q}[R] \cong A^{1 / q} \otimes_{A} R$ for all $q=p^{e}$, and hence $c R^{\infty} \subseteq A^{\infty}[R] \cong A^{\infty} \otimes_{A} R$ as well.

Proof. The natural map of $A^{1 / q} \otimes_{A} R$ into $R^{1 / q}$ has image $A^{1 / q}[R]$. Since the map becomes an isomorphism after localizing at the element $d$ and these modules are torsion-free over $A$, we see that $A^{1 / q} \otimes_{A} R \cong A^{1 / q}[R]$ for all $q$. By a direct limit argument, we see that we may identify $A^{\infty} \otimes_{A} R$ with $A^{\infty}[R] \subseteq R^{\infty}$ as well. Since $R^{1 / p} \supseteq A^{1 / p}[R]$ are finitely generated over $A^{1 / p}$ and become identical after localizing at $d$, it follows that there is a power $b$ of $d$ that multiplies $R^{1 / p}$ into $A^{1 / p}[R]$, as claimed. Let $h=1+1 / p+\cdots+1 / p^{e}$. We claim that $b^{h} R^{1 / p q} \subseteq A^{1 / p q}[R]$ for all $q=p^{e}$ by induction on $e$. We already know the case $e=0$ (where $h=1$ ) and taking $p$ th roots repeatedly yields $b^{1 / q} R^{1 / p q} \subseteq A^{1 / p q}\left[R^{1 / q}\right]$ for all $q$ whence, with $h^{\prime}=h-1 / q$, we have

$$
\begin{aligned}
b^{h} R^{1 / p q} & =b^{h^{\prime}} b^{1 / q} R^{1 / p q} \\
& \subseteq b^{h^{\prime}} A^{1 / p q}\left[R^{1 / q}\right] \\
& \subseteq A^{1 / p q}\left[b^{h^{\prime}} R^{1 / q}\right] \\
& \subseteq A^{1 / p q}\left[A^{1 / q}[R]\right]
\end{aligned}
$$


(since $b^{h^{\prime}} R^{1 / q} \subseteq A^{1 / q}[R]$ by the induction hypothesis) $=A^{1 / p q}$. Since $b^{h}$ divides $b^{2}$ (in $A^{1 / q}$ ) for all $h$, we have that $b^{2} R^{1 / q} \subseteq A^{1 / q}[R]$ for all $q$, as required, and the result for $R^{\infty}$ follows by taking the union.

We note that there is a different method for constructing elements $c$ as in Lemma (6.4). The two methods have different uses. The second is especially important for applications to finitely generated algebras over fields of characteristic 0 .

(6.5) Lemma. Let $R$ be module-finite, torsion-free, and generically smooth over a normal ring $A$. Let $r_{1}, \ldots, r_{d} \in R$ be a vector space basis for $L^{\prime}=L \otimes_{A} R$ over the fraction field $L$ of $A$, and let $c=\operatorname{det}\left(\operatorname{Tr}_{L^{\prime} / L}\left(r_{i} r_{j}\right)\right)$. Then $c \in A^{\circ}$ and $c R^{\infty} \subseteq A^{\infty}[R]$.

Proof. That $c$ is nonzero follows from the separability of $L^{\prime}$ over $L$. In fact, it is equivalent to the separability. It is clear that $c \in A$, since each trace is integral over $A$ and $A$ is normal. The fraction field of $A^{\infty}$ may be identified with $L^{\infty}$. Since $L^{\prime}$ is separable and $L^{\infty}$ purely inseparable over $L$, the $r_{i}$ are also a vector space basis for $L^{\prime \infty} \cong L^{\infty} \otimes_{L} L^{\prime}$ over $L^{\infty}$, and $c=\operatorname{det}\left(\operatorname{Tr}_{L^{\prime \infty} / L^{\infty}}\left(r_{i} r_{j}\right)\right)$ as well. Note also that $A^{\infty}$ is normal. The result now follows from the fact that $R^{\infty}$ is contained in the integral closure of $A^{\infty}$ in $L^{\prime \infty}$. Each element $u \in R^{\infty}$ has a unique representation as $\sum_{j=1}^{d} \lambda_{j} r_{j}$ with the $\lambda_{j}$ in $L^{\infty}$. Multiplying by $r_{i}$ and taking the trace yields a matrix equation $\left(\operatorname{Tr}\left(r_{i} r_{j}\right)\right)\left(\lambda_{i}\right)=\left(\operatorname{Tr}\left(u r_{i}\right)\right)$, and multiplying by the classical adjoint of $\left(\operatorname{Tr}\left(r_{i} r_{j}\right)\right)$ shows that each $c \lambda_{i}$ is in $A^{\infty}$, since all the traces are in $A^{\infty}$ (cf. the remark following the proof of Theorem 7 in Chapter V, $\S 4$ of [ZS, Vol. I]).

(6.6) Remarks on the norm. Let $A$ be a normal Noetherian domain and $R$ an algebra module-finite and torsion-free over $A$. Let $L$ denote the fraction field of $A$. Then $L \otimes_{A} R=S$ is a finite-dimensional $L$-vector space, and so we may define $\mathbf{N}=\mathbf{N}_{S / L}: S \rightarrow L$ to be the usual norm (i.e., $\mathbf{N}(s)=\operatorname{det}(s)$ viewed as a linear transformation on $S$ over $L)$. $\mathbf{N}(R) \subseteq A$ and, henceforth, in referring to $\mathbf{N}$ we always mean its restriction mapping $R$ to $A$. We may write $\mathbf{N}=\mathbf{N}_{R / A}$ to avoid ambiguity. Note that $r$ is a nonzerodivisor in $R$ iff $\mathbf{N}(r) \neq 0$, since tensoring with $L$ does not affect the issue. Also note that $r$ always divides $\mathbf{N}(r)$ in $R$, since, by the Cayley-Hamilton theorem, $r$ satisfies the characteristic poiynomial obtained by viewing multiplication by $r$ as a linear transformation on $S$, and the norm is the constant term of this polynomial. Thus, $r \in R^{\circ}$ iff $\mathbf{N}(r)$ is a nonzero multiple of $r$ in $A$ iff $r$ has a nonzero multiple in $A$.

Also note that $\mathbf{N}$ is multiplicative. If $R$ is reduced of positive characteristic $p$, then $\mathbf{N}$ extends uniquely to a multiplicative map $R^{\infty} \rightarrow A^{\infty}$ via the formula $\mathbf{N}(u)=\mathbf{N}\left(u^{q}\right)^{1 / q}$ for any $q \gg 0$. The result is independent of the choice of $q$. On $R^{1 / q}$, this agrees with the norm from $R^{1 / q}$ to $A^{1 / q}$.

(6.7) Remarks on base change and generic smoothness. Suppose that $R$ is module-finite, torsion-free, and generically smooth over a regular domain $A$ 
and that $A \subseteq B$, where $B$ is an $A$-flat regular domain. Then $B \otimes_{A} R$ is $B$ torsion-free, module-finite, and generically smooth over $B$. Note that $B \otimes_{A} R$ is consequently still reduced. Moreover, an element of $B \otimes_{A} R$ is in $\left(B \otimes_{A} R\right)^{\circ}$ iff it has a nonzero multiple in $B$, by (6.6). It follows that $R^{\circ} \subseteq\left(B \otimes_{A} R\right)^{\circ}$, whence $I^{*} \subseteq\left(I\left(B \otimes_{A} R\right)\right)^{*}$ for any ideal $I$ of $R$.

We also note that if $c \in A^{\circ}$ is such that $c R^{\infty} \subseteq A^{\infty}[R]$ and $S=B \otimes_{A}$ $R \supseteq B=B \otimes 1$, then $c S^{\infty} \subseteq B^{\infty}[S]$, since $S^{\infty}$ may be identified with $\left(B \otimes A_{R}\right)^{\infty} \cong B^{\infty} \otimes_{A^{\infty}} R^{\infty}$ and so is generated over $B^{\infty}$ by $R^{\infty}$ (i.e., $1 \otimes R^{\infty}$ ), and $c R^{\infty} \subseteq A^{\infty}[R]$. Thus, $c S^{\infty} \subseteq B^{\infty}[R] \subseteq B^{\infty}[S]$ (of course, since $S=$ $B \otimes_{A} R$, the last two terms are actually equal).

(6.8) Remarks on order. Let $(A, m)$ be a regular local ring. If $x \in A$, we define $\operatorname{ord}(x)=\operatorname{ord}_{m}(x)$ to be $\sup \left\{h \in \mathbf{N}: x \in m^{h}\right\}$ (which is $+\infty$ if $x=$ $0)$. If $A$ has characteristic $p$, we extend this to $A^{\infty}$ by defining ord $(u)$ as ord $\left(u^{q}\right) / q$ for any $q \gg 0$.

The following result permits us to test for tight closure using a fixed element $c$ independent of $I$; moreover, in certain cases it will allow us to pass to localizations and completions.

(6.9) Theorem. Let $R$ be torsion-free, module-finite, and generically smooth over a regular domain $A \subseteq R$. Let $c \in A^{\circ}$ be such that $c R^{\infty} \subseteq A^{\infty}[R]$. Then the following conditions on an ideal $I \subseteq R$ and an element $x \in R$ are equivalent.

(a) $x \in I^{*}$.

(b) For every maximal ideal $m$ of $A, x \in\left(I R_{m}\right)^{*}$.

(c) For every maximal ideal $m$ of $A, x \in\left(I \widehat{R}^{m}\right)^{*}$, where $\widehat{R}^{m}$ denotes the m-adic completion of $R$ and is isomorphic to $\widehat{A}_{m} \otimes_{A} R$, where $\widehat{A}_{m}$ denotes the completion of the local ring $A_{m}$ (which is isomorphic to the m-adic completion $\hat{A}^{m}$ of $A$ ).

$\left(c^{\circ}\right)$ For every maximal ideal $m$ of $R, x \in\left(I \widehat{R}_{m}\right)^{*}$, where $\widehat{R}_{m}$ denotes the completion of $R_{m}$.

(d) For every maximal ideal $m$ of $A$, there exists a sequence of elements $\left\{\varepsilon_{n}\right\}_{n}$ in $\left(\left(\widehat{R}^{m}\right)^{\infty}\right)^{\circ}$ such that $\operatorname{ord}\left(\mathbf{N}\left(\varepsilon_{n}\right)\right) \rightarrow 0$ as $n \rightarrow \infty$ and $\varepsilon_{n} x \in I\left(\widehat{R}^{m}\right)^{\infty}$. (N is the norm from $\left(\widehat{R}^{m}\right)^{\infty}$ to $\left(\hat{A}_{m}\right)^{\infty}$ described in (6.6); ord is the function described in (6.8).)

(e) $c x^{q} \in I^{[q]}$ for all $q$ of the form $p^{e}$.

Proof. We shall show that (a) $\Rightarrow(\mathrm{b}) \Rightarrow(\mathrm{c}) \Rightarrow(\mathrm{d}) \Rightarrow(\mathrm{e}) \Rightarrow(\mathrm{a})$ and (c) $\Leftrightarrow\left(c^{\circ}\right)$. (a) $\Rightarrow(b) \Rightarrow$ (c) is clear from the discussion in (6.7). Since the maximal ideals of $R$ are precisely the primes lying over maximal ideals $m$ of $A$ and since $\hat{A}_{m} \otimes_{A} R$ is the product of the completions of $R$ with respect to maximal ideals lying over $m$, the equivalence of $(c)$ and $\left(c^{\circ}\right)$ follows from Remark (4.13).

We next show that (c) $\Rightarrow(\mathrm{d})$. The key point is that if $x \in\left(I \widehat{R}^{m}\right)^{*}$, we have $d \in\left(\widehat{R}^{m}\right)^{\circ}$ such that $d x^{q} \in\left(I \widehat{R}^{m}\right)^{[q]}$ for all $q$, whence, as noted in (3.2), $d^{1 / q} x \in\left(I \widehat{R}^{m}\right)^{\infty}$ for all $q$. Now $\operatorname{ord}\left(\mathbf{N}\left(d^{1 / q}\right)\right)=(\operatorname{ord}(\mathbf{N}(d)))^{1 / q}=$ $\operatorname{ord}(\mathbf{N}(d)) / q$, which obviously $\rightarrow 0$ as $q \rightarrow \infty$, and so we may take $\varepsilon_{n}=$ $d^{1 / q}$ with $q=p^{n}$. Since (e) $\Rightarrow$ (a) trivially, we see that the only interesting 
implication is (d) $\Rightarrow(e)$, which asserts that one of the weakest conditions that might imply that an element is in the tight closure of an ideal is equivalent to one of the strongest such conditions.

Assume (d) but assume that there exists $e$ such that $c x^{q} \notin I^{[q]}$, where $q=p^{e}$. Then $c \notin I^{[q]}:{ }_{A} x^{q}$, and we can choose a maximal ideal $m$ of $A$ such that this is preserved when we tensor with $A_{m}$. Then, since $\widehat{A}_{m}$ is faithfully flat over $A_{m}$, the condition is preserved when we tensor with $\hat{A}_{m}$ as well. By (6.7), we may replace $A, R, I, x, c$ by their images after tensoring with $\hat{A}_{m}$. Henceforth, we change notation and assume that $A$ is complete local. Consider $\left\{\varepsilon_{n}\right\}_{n} \in\left(R^{\infty}\right)^{\circ}$ such that $\varepsilon_{n} x \in I R^{\infty}$ for all $n$ and $\operatorname{ord}\left(\mathbf{N}\left(\varepsilon_{n}\right)\right) \rightarrow 0$ as $n \rightarrow \infty$. Let $\delta=\delta_{n}=\mathbf{N}\left(\varepsilon_{n}\right)$. Then $\delta \in A^{\infty}$, and $\delta x \in I R^{\infty}$, since $\delta$ is a multiple of $\varepsilon_{n}$. Raising to the $q$ th power and multiplying by $c$ yields $\delta^{q}\left(c x^{q}\right) \in I^{[q]} c R^{\infty} \subseteq$ $I^{[q]} A^{\infty}[R]$. Now $A^{\infty}$ is flat over $A$, and so $A^{\infty}[R] \cong A^{\infty} \otimes_{A} R$ is flat over $R$. It follows that $\delta^{q} \in I^{[q]} A^{\infty}[R]: c x^{q}$ (over $\left.A^{\infty}[R]\right)=\left(I^{[q]}:{ }_{R} c x^{q}\right) A^{\infty}[R]$. But $R$ is a finite product of complete reduced local rings $\left(R_{i}, m_{i}\right)$ each of which is module-finite, torsion-free, and generically smooth over $A$. Since, by choice of $q, c x^{q} \notin I^{[q]}$, we can choose $i$ such that $I^{[q]} R_{i}: c x^{q} \subseteq m_{i}$. We then have that for every $n$ there is at least one choice of $i$ depending on $n$ such that $\delta_{n}^{q} \in m_{i} A^{\infty}\left[R_{i}\right]$. At least one $i$ must occur for infinitely many choices of $n$. Passing to a subsequence of the $\delta$ 's if necessary, we may assume that $\delta_{n}^{q} \in m_{i} A^{\infty}\left[R_{i}\right]$ for a fixed $i$ and all $n$. Let $P$ denote a minimal prime ideal of $R_{i}$. Then $P$ is disjoint from $A$, and the local domain $R_{i} / P=R^{\prime}$ with maximal ideal $m^{\prime}$ is module-finite, torsion-free, and generically smooth over $A$. We have an $A^{\infty}$-surjection of $A^{\infty}\left[R_{i}\right]$ to $A^{\infty}\left[R^{\prime}\right] \subseteq R^{\prime \infty}$, and this yields that $\delta_{n}^{q} \in m^{\prime} A^{\infty}\left[R^{\prime}\right]$ for all $n$. But by Lemma (6.10) below, this implies $\operatorname{ord}\left(\delta_{n}\right) \geq 1 / q d$ ! , where $d$ is the torsion-free rank of $R^{\prime}$ over $A$, contradicting $\operatorname{ord}\left(\delta_{n}\right) \rightarrow 0$ as $n \rightarrow \infty$.

(6.10) Lemma. Let $A$ be a local normal domain of characteristic $p$ and $R$ an extension domain module-finite and generically smooth over $A$, of torsion-free rank $d$ over $A$. Let $m$ be a proper ideal of $R$. Let $v$ be a discrete valuation of $A$ with values in $\mathbf{Z}$ that is positive on the maximal ideal of $A$. Extend $v$ to $A^{\infty}$ in the obvious way. Then for all $u \in m A^{\infty}[R] \cap A^{\infty}, v(u) \geq 1 / d$ !. If the extension of fraction fields is Galois, then $v(u) \geq 1 / d$.

Proof. We first consider the case where the fraction field $L^{\prime}$ of $R$ is Galois over the fraction field $L$ of $A$. Then $\left[L^{\prime}: L\right]=d$. Let $G$ be the Galois group. Let $x_{1}, \ldots, x_{n}$ be a set of generators for $m$ as an $A$-module. It follows that $u=$ $\sum_{i=1}^{h} a_{i} x_{i}$, where the $a_{i}$ are in $A^{\infty}$. Since $u$ and the $a_{i}$ are purely inseparable elements over $L$, we have that for each $g \in G, u=g(u)=\sum_{i=1}^{h} a_{i} g\left(x_{i}\right)$. Multiplying these equations together we obtain an equation of the form $u^{d}=$ $\sum_{\nu} a^{\nu} x_{\nu}$, where $\nu=\left(\nu_{1}, \ldots, \nu_{d}\right)$ runs through all $d$-tuples of nonnegative integers whose sum is $d, a^{\nu}=\prod_{t=1}^{d} a_{t}^{\nu_{t}}$, and $x_{\nu}$ denotes $\sum_{f} \prod_{g \in G} g\left(x_{f(g)}\right)$, 
where $f$ runs through all functions from $G$ to $\{1, \ldots, d\}$ which take on the value $i$ precisely $\nu_{i}$ times. It is easy to see that $x_{\nu}$ is an element of $L$ integral over $A$ and fixed by $G$. Since $A$ is normal, it is in $A$, hence in $m \cap A$, and so in the maximal ideal of $A$. It follows that $v\left(x_{\nu}\right) \geq 1$ for all $\nu$, and then since all of $u$, the $a^{\nu}$, and the $x_{\nu}$ are in $A^{\infty}$, we have that $d v(u) \geq 1$, as required.

In the general case, we choose a module-finite extension $S$ of $R$ such that the fraction field $L^{\prime \prime}$ of $S$ is Galois over $L$ and as small as possible. Then $\left[L^{\prime \prime}: L\right] \leq d !$. The result now follows from the Galois case.

(6.11) Remark. Theorem (6.9) remains valid if we replace ord in part (d) by any discrete valuation of the regular ring $A_{m}$ positive on $m$. The proof goes through without change.

(6.12) Remark. We want to present a different perspective on tight closure. Suppose for simplicity that $R$ is reduced. Then $x \in I^{*}$ iff $\bigcap_{q}\left(I^{[q]}:{ }_{R} x^{q}\right)$ meets $R^{\circ}$. When $R$ is module-finite, torsion-free, and generically smooth over a regular domain $A$, this is equivalent to asserting that $\bigcap_{q}\left(I^{[q]}:{ }_{R} x^{q}\right)$ meets $A^{\circ}$. One of the main points of Theorem (6.9) is that it suffices to check an enormously weaker (on the face of it) condition, that locally on $A$ there is an element $\delta_{n} \in\left(I^{[q]}:{ }_{R} x^{q}\right) \cap A^{\circ}$ of relatively low order as $q=p^{n}$ grows, in the sense that $\operatorname{ord}\left(\delta_{n}\right) / q \rightarrow 0$ as $n \rightarrow \infty$.

(6.13) Theorem. Let $R$ be module-finite, torsion-free, and generically smooth over a regular domain $A$ of characteristic $p$. Then every element $d \in A^{\circ}$ such that $R_{d}$ is smooth over $A_{d}$ has a power $c$ in the ideal of test elements which, in fact, is a completely stable test element in $B \otimes_{A} R$ for every A-flat regular domain $B \supseteq A$. A sufficient condition for $c$ to have this property is that $c R^{\infty} \subseteq A^{\infty}[R]$. In particular, $c$ is a completely stable test element in every polynomial ring over $R$.

Proof. Except for the last statement, this is clear from Theorem (6.9). All hypotheses are preserved when we pass from $A$ to $B$. We can assume $B=A$. Given any prime ideal of $R$, it lies over a prime ideal of $A$. We may localize to reduce to the case where $A$ is local and the prime of $R$ is maximal. Local stability then follows from Proposition (6.1). Similarly, we may use Theorem (6.9) to pass to the completion of $A$, and then complete stability follows from Proposition (6.1). The statement about polynomial rings follows because $R[x]$, where $x$ may represent several indeterminates, is $A[x] \otimes_{A} R$, and so we may apply the result on base change.

The situation is still quite good if $R$ is reduced, torsion-free, and modulefinite over a regular domain $A$ provided that $A$ is excellent. Difficulties arise when $A$ is not excellent, since there are, for example, DVR's $A$ such that $\hat{A}$ is purely inseparable over $A$ (see [N, Appendix A1, (E3.3)]). We make a remark and then prove two lemmas that enable us to deduce our main result, Theorem (6.17) below, from the generically smooth case.

(6.14) Remark. If $h: R \rightarrow S$ is a homomorphism of reduced rings of characteristic $p$, then $h$ extends uniquely to a map from $R^{1 / q}$ to $S^{1 / q}$ by $h\left(r^{1 / q}\right)=$ $h(r)^{1 / q}$. More generally, if $W$ is any subset of $R$, then $h$ extends uniquely 
to a homomorphism from $R\left[w^{1 / q}: w \in W\right]$ to $S^{\prime}$, where $S \subseteq S^{\prime} \subseteq S^{1 / q}$, provided that $S^{\prime}$ contains a $q$ th root of every element in $h(W)$.

(6.15) Lemma. Let $R$ be module-finite, torsion-free, and reduced over a regular ring $A$ of characteristic $p$.

(a) For all sufficiently large $q, R\left[A^{1 / q}\right]$ is module-finite, torsion-free, and generically smooth over $A^{1 / q}$.

(b) Suppose that $A$ is excellent and local as well and that $q$ has been chosen so that the conclusion of (a) holds. Let $A^{\prime}=A^{1 / q}$. Then there is a natural isomorphism between $S=\left(A^{\prime}\right)^{\wedge} \otimes_{A^{\prime}} R\left[A^{\prime}\right]$ and $T=\left(\hat{A} \otimes_{A} R\right)\left[\hat{A}^{1 / q}\right]$, where $R\left[A^{\prime}\right]$ is to be interpreted as a subring of $R^{1 / q}$ and $T$ is to be interpreted as $a$ subring of $\left(\widehat{A} \otimes_{A} R\right)^{1 / q}$.

Proof. (a) is obvious. For generic smoothness we need only consider what happens after tensoring with the fraction field of $A$. But then $R$ becomes a product of fields and the field case is well known.

To prove (b), we first note that there is an identification of $\left(A^{\prime}\right)^{-}$(which is $\left.\left(A^{1 / q}\right)^{\wedge}\right)$ with $(\widehat{A})^{1 / q}$. Call this ring $B$. Both $S$ and $T$ are module-finite over $B$, generated as $B$-modules by the image of $R$ (or any set of generators for $R$ as an $A$-module). We shall exhibit $B$-algebra maps between them in both directions, each of which is clearly the identity on the image of $R$. The result is then immediate.

A crucial point is that both $S$ and $\widehat{A} \otimes_{A} R$ are reduced. The former holds because $R\left[A^{\prime}\right]$ is generically smooth over $A^{\prime}$ and the latter because $A$ is excellent and $R$ is reduced (since $A \rightarrow \widehat{A}$ is flat with smooth fibers, so is $R \rightarrow \widehat{A} \otimes_{A} R$ ). The fact that $\hat{A} \otimes_{A} R$ is reduced is needed to view $\left(\hat{A} \otimes_{A} R\right)^{1 / q}$ as an extension of $\hat{A} \otimes_{A} R$. Note that $T$ is consequently reduced as well.

To give the map from $S$ to $T$, it suffices to given an $A^{\prime}$-algebra map from each factor in the tensor product. It is clear how to do this for the first factor. For the second factor we use Remark (6.14). We have an obvious injection of $R$ into $\widehat{A} \otimes_{A} R$ and hence into $T$, and the map extends to $R\left[A^{1 / q}\right]$ because each element of $A$ has a $q$ th root in $T$.

Similarly, we have an obvious map of $\hat{A} \otimes_{A} R$ into $S$, and the map extends to $T$ because the image of each element of $\hat{A}$ has a $q$ th root in $S$.

(6.16) Lemma. Let $R$ be a reduced Noetherian ring of characteristic $p$, and let $S$ be a Noetherian ring such that $R \subseteq S \subseteq R^{1 / q^{\prime}}$. Suppose that $c \in S$ is $a q^{\prime \prime}$-weak test element for $S$. Then $c^{q^{\prime}}$ is a $\left(q^{\prime} q^{\prime \prime}\right)$-weak test element for $R$. If $c$ is a locally stable $q^{\prime \prime}$-iveak test element for $S$, then $c^{q^{\prime}}$ is a locally stable $\left(q^{\prime} q^{\prime \prime}\right)$-weak test element for $R$.

Proof. If $x \in I^{*}$ in $R$, we have $x \in(I S)^{*}$, and so $c x^{q} \in I^{[q]} S \subseteq I^{[q]} R^{1 / q^{\prime}}$ for all $q \geq q^{\prime \prime}$. Raising to $q^{\prime}$ th powers yields $c^{q^{\prime}} x^{q^{\prime} q} \in I^{\left[q^{\prime} q\right]}$ for all $q \geq q^{\prime \prime}$, and 
$\left\{q^{\prime} q: q \geq q^{\prime \prime}\right\}$ is the same as $\left\{q: q \geq q^{\prime} q^{\prime \prime}\right\}$. The locally stable case follows at once by localization.

(6.17) Theorem. Let $R$ be a reduced ring that is module-finite and torsion-free over a locally excellent regular domain $A$ of characteristic $p$. If $q^{\prime}$ is sufficiently large so that $R\left[A^{1 / q^{\prime}}\right]$ is generically smooth over $A^{1 / q^{\prime}}$ and $c \in A^{\circ}$ is such that $c^{1 / q^{\prime}} R^{\infty} \subseteq A^{\infty}[R]$ (such elements always exist), then $c$ is a completely stable $q$ '-weak test element in $B \otimes_{A} R$ for every locally excellent regular A-flat domain $B \supseteq A$ such that $B \otimes_{A} R$ is reduced (this last condition holds, for example, if the generic fiber of $A \rightarrow B$ is geometrically reduced, i.e., for every map of $A$ to a finite algebraic extension field $L$ of its fraction field, $L \otimes_{A} B$ is reduced). In particular, $c$ is a completely stable $q^{\prime}$-weak test element in every polynomial ring over $R$ (taking $B=A\left[x_{1}, \ldots, x_{h}\right]$ ).

Proof. Note that $R\left[A^{1 / q^{\prime}}\right]^{\infty}=R^{\infty}$. We apply Theorem (6.13) to construct $c^{1 / q^{\prime}}$ in $A^{1 / q^{\prime}}$. The hypotheses are unaffected by applying $B \otimes_{A}$, and so we might as well assume $B=A$. We can localize at any prime of $A$. Once $A$ is local, with $A^{\prime}=A^{1 / q^{\prime}}$, we have that $c^{1 / q^{\prime}}$ is a test element in $\left(A^{1 / q}\right)^{\wedge} \otimes_{A^{\prime}} R\left[A^{\prime}\right]$, which, by Lemma (6.15), we may identify with a subring of $\left(\widehat{A} \otimes_{A} R\right)^{1 / q^{\prime}}$. We may then apply Lemma (6.16) to conclude that $c$ is a $q^{\prime}$-weak test element for $\widehat{A} \otimes_{A} R$, which shows that $c$ is completely stable for the original ring $R$.

(6.18) Corollary. Let $R$ be a reduced and locally equidimensional algebra of characteristic $p$ that is essentially of finite type over a field $K$. Then $R$ has a $q^{\prime}$-weak test element $c$ that is a completely stable $q^{\prime}$-weak test element in $B \otimes_{K} R$ for every locally excellent regular domain $B \supseteq K$ such that $B \otimes_{K} R$ is reduced.

Proof. If $\operatorname{Spec} R$ has more than one component we can solve the problem on each component separately. Therefore, we may assume that $\operatorname{Spec} R$ is connected. We can represent $R$ as a localization of a reduced finitely generated $K$-algebra $S$ at a multiplicative system $U$, and by localizing $S$ at finitely many elements we may also assume that $\operatorname{Spec} S$ is connected and that the minimal primes of $S$ correspond bijectively with those of $R=U^{-1} S$. We can also assume that $B \otimes_{K} S$ is reduced (localizing enough to kill the nilpotents).

We next claim that for any two minimal primes $P, P^{\prime}$ of $S, \operatorname{dim} S / P=$ $\operatorname{dim} S / P^{\prime}$. To see this, note that there is a sequence of primes $P R, Q_{1} R$, $P_{2} R, \ldots, Q_{h} R, P^{\prime} R$ of $R$, where each $P_{j}, Q_{j}$ is prime in $S$, such that

$$
P=P_{1} \subseteq Q_{1} \supseteq P_{2} \subseteq Q_{2} \supseteq P_{3} \subseteq \cdots \subseteq Q_{n} \supseteq P_{h}=P^{\prime},
$$

simply because $\operatorname{Spec} R$ is connected. (The $P$ 's and $Q$ 's alternate, as do the directions of the symbols $\subseteq, \supseteq$.) To complete the proof that $\operatorname{dim} S / P=$ $\operatorname{dim} S / P^{\prime}$, it suffices to show that if $P_{j} \subseteq Q \supseteq P_{j+1}$ and $Q$ does not meet $U$, then $\operatorname{dim} R / P_{j}=\operatorname{dim} R / P_{j+1}$. The point is that since $U^{-1} R$ is locally equidimensional, we must have ht $Q R / P_{j} R=\mathrm{ht} Q R / P_{j+1} R$, which implies that ht $Q / P_{j}=$ ht $Q / P_{j+1}$ in $S$. Choose a maximal ideal $m$ of $S$ that contains 
$Q$. Since $S$ is catenary, ht $m / P_{j}=\mathrm{ht} m / P_{j+1}$. Since all maximal ideals of a domain finitely generated over a field have the same height, we get $\operatorname{dim} S / P_{j}=$ ht $m / P_{j}=$ ht $m / P_{j+1}=\operatorname{dim} S / P_{j+1}$. We may therefore assume that for every maximal ideal $m$ of $S$ and every minimal prime $P$ of $S$ contained in $m$, ht $m / P=\operatorname{dim} S$, i.e., that $S$ is biequidimensional.

It will suffice to prove the result for the biequidimensional ring $S$, for any completion of a local ring of $B \otimes_{K} U^{-1} S \cong U^{-1}\left(B \otimes_{K} S\right)$ is evidently a completion of a local ring of $B \otimes_{K} S$.

By Noether normalization, $S$ is a module-finite extension ring of a polynomial ring $A=K\left[x_{1}, \ldots, x_{n}\right]$. The fact that $S$ is biequidimensional implies that it is torsion-free as an $A$-module. Since $B$ is flat over $K$, the regular ring $B^{\prime}=B\left[x_{1}, \ldots, x_{n}\right]$ is flat over $A$. We may now apply Theorem (6.17) to conclude that there is an element of $S$ that is a completely stable $q^{\prime}$-weak test element for $B^{\prime} \otimes_{A} S \cong B \otimes_{K} S$.

Similarly,

(6.19) Corollary. Let $R$ be a reduced, equidimensional, complete local ring of characteristic $p$. Then $R$ has a completely stable $q^{\prime}$-weak test element $c$.

We conclude this section with a sequence of results that will enable us to deduce the existence of test elements from the existence of weak test elements in several important instances.

We begin with

(6.20) Proposition. Let $R$ be a reduced ring of characteristic $p$, and suppose that $c^{\prime}$ is a $q^{\prime}$-weak test element for $R$. Suppose there is an $R$-linear map $R^{1 / p} \rightarrow R$ that takes on a value $c^{\prime \prime} \in R^{\circ}$. Then $c^{\prime} c^{\prime \prime 2}$ is a test element for $R$.

Moreover, if $c^{\prime}$ is a locally (respectively, completely) stable $q^{\prime}$-weak test element, then $c^{\prime} c^{\prime \prime 2}$ is a locally (respectively, completely) stable test element.

Proof. By Lemma (6.21) below, we can construct an $R$-linear map $g: R^{1 / q^{\prime}} \rightarrow$ $R$, whose value on 1 is $c^{\prime \prime 2}$. Now suppose that $x \in I^{*}$ in $R$. Then $c^{\prime} x^{q q^{\prime}} \in$ $I^{q q^{\prime}}$ for all $q$. Hence, $\left(c^{\prime} x^{q}\right)^{q^{\prime}} \in\left(I^{[q]}\right)^{\left[q^{\prime}\right]}$, which shows that $c^{\prime} x^{q} \in I^{[q]} R^{1 / q^{\prime}}$ when we take $q^{\prime}$ th roots. Applying the given $R$-linear $g$ then yields that $c^{\prime} c^{\prime \prime 2}$ is a test element.

The final statement is a consequence of the fact that both $c^{\prime}$ and $c^{\prime \prime}$ will retain their respective properties upon localization (respectively, localization and completion) in the locally (respectively, completely) stable case.

(6.21) Lemma. Let $R$ be a reduced ring of characteristic $p$, and suppose there is an $R$-linear map $f: R^{1 / p} \rightarrow R$ such that $f(u)=c \in R^{\circ}$ for some element $u \in R^{1 / p}$. Then there is an $R$-linear map $g_{q}: R^{1 / q} \rightarrow R$ such that $g_{q}(1)=c^{2}$.

Proof. We use induction on $e$, where $q=p^{e}$. If $e=0$, this is obvious, and if $e=1$, we let $g_{p}(v)=c f(u v)$. To complete the induction, suppose we have such a map from $R^{1 / q} \rightarrow R$. Taking $p$ th roots gives an $R^{1 / p}$-linear map 
from $h: R^{1 / p q} \rightarrow R^{1 / p}$ such that $h(1)=c^{2 / p}$. Since $2 / p \leq 1$, we may let $g_{p q}(w)=f\left(c^{1-(2 / p)} h(w) u\right)$ for all $w \in R^{1 / p q}$.

Before giving the next result, we should remark that $A^{1 / p}$ is not assumed to be module-finite over $A$. This is what makes the issue nontrivial.

(6.22) Proposition. Let $A$ be a reduced ring of characteristic $p$ and $R$ a reduced module-finite extension of $A$, torsion-free over $A$. Suppose that there exists an A-linear map $f: A^{1 / p} \rightarrow A$ taking on a value in $A^{\circ}$. Then there exists an $R$-linear map $h: R^{1 / p} \rightarrow R$ such that $h(1) \in A^{\circ}\left(\subseteq R^{\circ}\right)$.

Proof. If $f(u)=a$, we may replace $f$ by the map $f^{\prime}$, where $f^{\prime}(z)=f(u z)$. Thus, we may assume that $f(1)=a \in A^{\circ}$. Note that the torsion-free property for $A \subseteq R$ (and the fact that the rings are reduced) implies that $A^{\circ} \subseteq R^{\circ}$. Note also that the total quotient ring $A^{\prime}=\left(A^{\circ}\right)^{-1} A$ is a finite product of fields, and hence that $A^{\prime} \otimes_{A} \operatorname{Hom}_{A}(R, A) \cong A^{\prime} \otimes_{A} R$, since, locally on $A^{\prime}, A^{\prime}$ is a field and $A^{\prime} \otimes_{A} R$ is a finite product of fields. Let $Q_{1}, \ldots, Q_{s}$ be the minimal primes of $R$. Let $e_{1}, \ldots, e_{s}$ be the idempotents of $R^{\prime}=A^{\prime} \otimes_{A} R$ such that $e_{j}$ is in all the $Q_{t}$ except $Q_{j}$. For each minimal prime $P_{i}$ of $A$, let $f_{i}$ be the sum of the $e_{j}$ such that $Q_{j}$ lies over $P_{i}$. Let $i(j)$ denote the integer such that $Q_{j}$ lies over $P_{i(j)}$. Choose $a^{\prime} \in A^{\circ}$ such that $a^{\prime} e_{j} \in R$ for every $j$ and $a^{\prime} f_{i} \in A$ for every $i$. It is easy to see that there is an $A^{\prime}$-linear map $g: R^{\prime} \rightarrow A^{\prime}$ whose value on $e_{j}$ is $f_{i(j)}$ for every $j$. This condition insures that $g$ is a generator of $\operatorname{Hom}_{A^{\prime}}\left(R^{\prime}, A^{\prime}\right)$. In fact, it is sufficient (but still not necessary) that for all $j$, $g\left(e_{j}\right)$ is $\alpha_{j} f_{i(j)}$ for some $\alpha_{j} \in A^{\prime 0}$ for $g$ to be such a generator. Now choose $a^{\prime \prime} \in A^{\circ}$ such that $a^{\prime \prime} g(R) \subseteq A$. Let $g^{\prime}=\left.a^{\prime \prime p} g\right|_{R}$. Then $g^{\prime}: R \rightarrow A$.

Taking $p$ th roots, we obtain a map $g^{1 / p}: R^{1 / p} \rightarrow A^{1 / p}$ that is $A^{1 / p}$-linear. Its value on $a^{\prime} e_{j}$ is

$$
\begin{aligned}
\left(a^{\prime \prime p} g\left(\left(a^{\prime} e_{j}\right)^{p}\right)\right)^{1 / p} & =\left(\left(a^{\prime \prime p} g\left(a^{\prime p} e_{j}\right)\right)\right)^{1 / p} \\
& =\left(a^{\prime \prime p} a^{\prime p} f_{i}\right)^{1 / p} \\
& =a^{\prime \prime} a^{\prime} f_{i}
\end{aligned}
$$

Composing with $f$ yields a map $\phi: R^{1 / p} \rightarrow A$ that sends $a^{\prime} e_{j}$ to $a a^{\prime} a^{\prime \prime} f_{i}$ and is $A$-linear. Applying $\operatorname{Hom}_{A}(, A)$ to the inclusion $R \subseteq R^{1 / p}$ gives an $R$-linear map $\theta: \operatorname{Hom}_{A}\left(R^{1 / p}, A\right) \rightarrow \operatorname{Hom}_{A}(R, A)$. We then define $\lambda: R^{1 / p} \rightarrow$ $\operatorname{Hom}_{A}(R, A)$ by $\lambda(r)=\theta(r \phi)$. The map $\lambda$ is $R$-linear and $\lambda(1)=\left.\phi\right|_{R}$ is a map from $R$ to $A$ whose value on $a^{\prime} e_{j}$ is $a a^{\prime} a^{\prime \prime} f_{i}$. It follow that $\lambda(1)$ is a generator of $A^{\prime} \otimes_{A} \operatorname{Hom}_{A}(R, A) \cong R^{\prime}$ as an $R^{\prime}$-module. We can choose an $R^{\prime}$-linear isomorphism $\gamma: A^{\prime} \otimes_{A} \operatorname{Hom}_{A}(R, A) \cong R^{\prime}$ that sends $\lambda(1)$ to 1 . If we multiply by a suitable element $b$ of $A^{\circ}$, we have $b \gamma\left(\operatorname{Hom}_{A}(R, A)\right) \subseteq R$. But then $b \gamma \lambda: R^{1 / p} \rightarrow R$ is $R$-linear and maps 1 to $b$. 
(6.23) Proposition. Suppose that $R$ is reduced and is module-finite and torsionfree over a regular domain $A$. Suppose that $A^{1 / p} \rightarrow A$ splits. Then $R$ has a completely stable test element.

Proof. We already know that $R$ has a completely stable weak test element. By Proposition (6.22), we may apply Proposition (6.20) to conclude that $R$ has a completely stable test element.

(6.24) Corollary. Let $R$ be reduced of characteristic $p$ and locally equidimensional. Suppose that either $R$ is essentially of finite type over a field or that $R$ is complete local. Then $R$ has a completely stable test element.

Proof. If $R$ is complete local, it is module-finite over $A$, where $A$ is complete regular local. It suffices to observe that $A \rightarrow A^{1 / p}$ splits. It is pure, since $A^{1 / p}$ is faithfully flat over $A$. The equidimensionality implies that it is torsion-free over $A$.

The following argument, which is due to $M$. Auslander, shows that a pure injection $A \rightarrow M$ of a complete local ring $A$ into an arbitrary module $M$ splits. Let $E$ be the injective hull of the residue field of $A . A \otimes_{A} E \rightarrow M \otimes_{A} E$ is injective, by the purity. Since $E$ is an injective module, $\operatorname{Hom}_{A}(M \otimes E, E) \rightarrow$ $\operatorname{Hom}_{A}(A \otimes E, E)$ is surjective. By the adjointness of $\otimes$ and Hom, we may identify this map with the map

$$
\operatorname{Hom}_{A}\left(M, \operatorname{Hom}_{A}(E, E)\right) \rightarrow \operatorname{Hom}_{A}\left(A, \operatorname{Hom}_{A}(E, E)\right) \text {. }
$$

Since $A$ is complete, $A \rightarrow \operatorname{Hom}(E, E)$ is an isomorphism, and so

$$
\operatorname{Hom}_{A}(M, A) \rightarrow \operatorname{Hom}_{A}(A, A)
$$

is surjective. Thus, the identity on $A$ lifts to a map from $M$ to $A$, as required.

As in the proof of (6.18), when $R$ is locally equidimensional and essentially of finite type over a field, we can work with each connected component of Spec $R$ separately, and in the connected case we know that $R$ is a localization of a biequidimensional reduced ring over $K$. Thus, the question reduces to the case where $R$ is biequidimensional and of finite type over a field $K$. Then $R$ is module-finite over a polynomial ring $A=K\left[x_{1}, \ldots, x_{n}\right]$, and it suffices to observe that $A \rightarrow A^{1 / p}$ is a free extension, and so splits: it is the composite of the extension $A \rightarrow K^{1 / p}\left[x_{1}, \ldots, x_{n}\right]$, which is free because $K^{1 / p}$ is $K$-free, and $K^{1 / p}\left[x_{1}, \ldots, x_{n}\right] \subseteq K^{1 / p}\left[x_{1}^{1 / p}, \ldots, x_{n}^{1 / p}\right]$, which is also free.

The next observation can be used to simplify the treatment of test elements in this section but was only noticed by the authors at a late stage in the preparation of the manuscript. Thus, we were not able to incorporate it into the main body of this section, and exploit it fully. We note that the result $(6.25 \mathrm{~b})$ on test elements for ideals works equally well, without essential modification in the proof, for test elements for modules (working with submodules of free modules in place of ideals). See $\S 8$.

(6.25) Proposition. Let $R$ be a Noetherian ring of characteristic $p$ with minimal primes $P_{1}, \ldots, P_{h}$. Let $N=\bigcap_{i} P_{i}$ be the ideal of all nilpotent elements in $R$. Let $q^{\prime \prime}$ be such that $N^{\left[q^{\prime \prime}\right]}=(0)$. 
(a) An element $x \in R$ is in the tight closure $I^{*}$ of an ideal $I \subseteq R$ if and only if the image of $x$ in $R / P_{i}$ is in the tight closure of $I R / P_{i}$ (over $R / P_{i}$ ) for each $i$.

(b) If each of the rings $R / P_{i}$ has a $q^{\prime}$-weak test element, then $R$ has a $q^{\prime} q^{\prime \prime}$ weak test element. Hence, if $R$ is reduced and each $R / P_{i}$ has a test element, then $R$ has a test element.

Proof. First note that if $\gamma_{i} \in R / P_{i}-\{0\}$, then there exists $c_{i} \in R^{\circ}$ which maps to $\gamma_{i}$ : if $d$ is any lifting, we cannot have $d+P_{i} \subseteq \bigcup_{j} P_{j}$ unless $R d+P_{i}$ is contained in one of the $P_{j}$, by [Kap, Theorem 124, p. 90]. For the "if" part of (a) ("only if" is clear), suppose that $\gamma_{i} \in R / P_{i}-\{0\}$ for each $i$ is such that $\gamma_{i} \bar{x}^{q} \in\left(I R / P_{i}\right)^{[q]}$ for all $q \geq q^{\prime}$, i.e., such that for all $q \geq q^{\prime}, c_{i} x^{q} \in I^{[q]}+P_{i}$, where $c_{i}$ is a lifting of $\gamma_{i}$ to $R^{\circ}$. Let $\lambda_{i} \in R$ be in all the $P_{j}$ except $P_{i}$. Then $\lambda_{i} P_{i} \subseteq N$, so that for each $i$ and all $q \geq q^{\prime}, \lambda_{i} c_{i} x^{q} \in I^{[q]}+N$. It follows that $\left(\Sigma_{i} \lambda_{i} c_{i}\right)^{q^{\prime \prime}} x^{q q^{\prime \prime}} \in I^{\left[q q^{\prime \prime}\right]}$ for all $q \geq q^{\prime}$, and it is easy to see that $\Sigma_{i} \lambda_{i} c_{i} \in R^{\circ}$, and hence $c=d^{q^{\prime \prime}} \in R^{\circ}$. The proof of (b) is identical except that we pick $\gamma_{i}$ to be a $q$-weak test element for $R / P_{i}$ (independent of the choice of $x$ and $I$ ) and then observe that the argument for (a) shows that $c=\left(\Sigma_{i} \lambda_{i} c_{i}\right)^{q^{\prime \prime}}$, where $c_{i}$ lifts $\gamma_{i}$ to $R^{\circ}$ and the $\lambda_{i}$ are as above, is a $q^{\prime} q^{\prime \prime}$-weak test element for $R$.

(6.26) Corollary. Let $R$ be a ring of characteristic $p$ and suppose that $R$ is essentially of finite type over a field or else that $R$ is a complete local ring. Then $R$ has a $q^{\prime}$-weak test element. If $R$ is reduced, then $R$ has a test element.

\section{CONSTRAINTS ON SYSTEMS OF PARAMETERS IN CHARACTERISTIC $p$}

It has already been noted that when $R$ is a module-finite and torsion-free overring of a regular ring $A$ in characteristic $p$ and $x_{1}, \ldots, x_{n}$ are elements of $A$ that are parameters in $R$, then we have

$$
\left(x_{1}, \ldots, x_{n-1}\right) R:{ }_{R} x_{n} \subseteq\left(\left(x_{1}, \ldots, x_{n-1}\right) R\right)^{*} .
$$

In this section, we prove several generalizations of this result. See particularly Theorems (7.9) and (7.15). For example, the result is valid in locally equidimensional homomorphic images of a Cohen-Macaulay ring. It is not necessary to assume that $R$ is module-finite over a regular ring. (In [HH4], we show that related results on Koszul homology hold with the local equidimensionality condition relaxed, provided that more is assumed about the sequence of elements.) There are similar results for ideals generated by monomials in parameters. There are related but incomparable results in several other contexts.

We shall also see that there are comparable results for ideals constructed by iterated use of the addition, multiplication, intersection, and colon operations on ideals generated by monomials in parameters and, likewise, on the expansions of arbitrary ideals from a regular ring $A$ under a map $h: A \rightarrow R$ under certain conditions on $A, R$, and $h$. See Remark (7.2) below and the multipart monster, Theorem (7.15), which details numerous situations in which results on 
iterated operations on ideals are valid. One always needs to assume, in effect, that the heights of certain ideals of $A$ do not decrease when they are expanded to $R$ in order to obtain such results.

Unless otherwise noted, all rings in this section are assumed to have positive prime characteristic $p$. In [HH7], the results proved here are extended to characteristic 0 .

It is worth pointing out that every result we prove here to the effect that a certain colon ideal (or other result of an operation or sequence of operations) is in the tight closure of a certain ideal implies that it is in the integral closure. However, for sequences of operations, it is hopeless to try to prove a result of this type working entirely with integral closures. The integral closure that replaces the expected answer gets much too big, and one loses so much information that it is hopeless to carry through. For example, our results imply that for parameters $x, y$, under mild conditions on the ring,

$$
\left(\left(x^{2}, y^{3}\right) R \cap\left(x^{3}, y^{2}\right) R\right):\left(x y^{2} R\right) \subseteq((x, y) R)^{*} \subseteq((x, y) R)^{-} .
$$

If one approaches this by trying to use the fact that the given intersection is in the integral closure of the expected answer, one only obtains that the intersection is in $\left(\left(x^{3}, y^{3}, x^{2} y^{2}\right) R\right)^{-}$. Since this integral closure contains $x y^{2}$, one gets no information about the colon.

(7.1) Remark. It is worth noting that for every set of hypotheses for which we can prove results on capturing the colon, we also obtain the result that F-regular rings that satisfy those hypotheses are Cohen-Macaulay.

(7.2) Remark. We want to explain the close relationship between the results on ideals generated by monomials in parameters and the results on ideals expanded from regular rings. What one hopes is that if $A$ is regular and $A \rightarrow R$ is such that for all the ideals $I$ of $A$ in a certain family $\mathscr{I}$ one has that height $I R \geq$ height $I$ (this is true when $R$ is a module-finite overring of $A$, but also when $R$ is flat and in many other situations), then for all $I, J \in \mathscr{I}$ one has that $I R:{ }_{R} J R \subseteq\left(\left(I:{ }_{A} J\right) R\right)^{*}$ and that $I R \cap J R \subseteq((I \cap J) R)^{*}$. However, we can prove such results only after imposing special hypotheses of one sort or another. The situation is better when dealing with ideals generated by monomials in parameters. One useful point is that, after localization, completion, and possibly certain other manipulations, such as enlarging the set of parameters, ideals generated by monomials in parameters $x_{1}, \ldots, x_{n}$ can be viewed as arising by expansion from a regular ring $K\left[\left[x_{1}, \ldots, x_{n}\right]\right]$ to a module-finite extension. Unfortunately, many technical complications arise in trying to carry through a program of deducing the results about monomials from this case, partly because subfields of a complete local ring do not always extend to coefficient fields in characteristic $p$, and partly because, in working with tight closure, it is necessary to assume the existence of completely stable test elements of some sort in order to be able to reduce to the complete local case. We consequently are forced to do a certain amount of proving of parallel results in order to get the theorems we want. For example, although we can prove that ideals generated 
by monomials in parameters behave well in any locally equidimensional quotient of a Cohen-Macaulay ring, we cannot obtain the result in this generality by localizing and completing. We need to use a quite different technique.

The rest of this section is structured as follows. We first develop the material we need to obtain our results on the behavior of the colon and intersection operations for monomial ideals in locally equidimensional homomorphic images of Cohen-Macaulay rings. (The equidimensionality condition is not needed if one imposes height conditions modulo every minimal prime. We discuss this in [HH4]. The context is somewhat different, however.) Theorem (7.9) is the first culmination of this development. We then prove, in a very general form, the key lemma we need, Theorem (7.12), to obtain results on iterated operations. We can then combine Theorem (7.12) succesively with Theorems (7.9) and (4.8) and results of $\S 6$ to obtain several situations in which our results on iterated operations are valid. See Theorem (7.15).

(7.3) Discussion. Throughout this discussion and its sequel, we drop the restriction to rings of characteristic $p$, imposing it again when we come to Theorem (7.9).

Let $x_{1}, \ldots, x_{n}$ be elements of the ring $R$. By a monomial in the $x_{i}$ we mean an expression of the form $x^{\nu}$, where $\nu$ is an $n$-tuple of nonnegative integers $\left(\nu_{1}, \ldots, \nu_{n}\right)$ and $x^{\nu}=\prod_{i=1}^{n} x_{i}^{\nu_{i}}$. In the polynomial ring $\Lambda\left[X_{1}, \ldots, X_{n}\right]$, where $\Lambda$ is any base ring, the sum, product, intersection, and colon of two ideals generated by monomials in the $X_{i}$ can be computed by formal rules, and the result is again an ideal generated by monomials. Suppose that each of the ideals $I, J$ has a fixed finite set of monomial generators. Call these $B, C$. Then $I+J$ is generated by $B \cup C, I J$ by $B C=\{b c: b \in B$ and $c \in C\}$, and $I \cap J$ by $\{\operatorname{LCM}(b, c): b \in B$ and $c \in C\}$. Note that if $b=x^{\mu}$ and $c=x^{\nu}$, then $\operatorname{LCM}(b, c)=x^{\max \{\mu, \nu\}}$, where the $i$ th entry of $\max \{\mu, \nu\}$ is $\max \left\{\mu_{i}, \nu_{i}\right\}$. A similar rule may be used for the calculation of monomial generators of the intersection of any finite number of ideals. If $J$ is generated by a single monomial $c=x^{\nu}$, then $I: J$ is generated by the elements $x^{\mu-\nu}$, where $x^{\mu}$ runs through a set of monomial generators for $I$ and $\mu \dot{-} \nu$ has as its $i$ th entry $\mu_{i} \dot{-} \nu_{i}=\max \left\{\mu_{i}-\nu_{i}, 0\right\}$. In general, we may compute $I: J$ as $\bigcap_{c \in C} I:(c)$. Cf. [EHo, Tay]. In fact, the formal rules described above are valid not only for indeterminates over $\Lambda$, but also for any permutable possibly improper regular sequence in any commutative ring. (Elements $x_{1}, \ldots, x_{n} \in R$ form a possibly improper regular sequence if for every $i, 0 \leq i \leq n-1$, $\left(x_{1}, \ldots, x_{i}\right) R:{ }_{R} x_{i+1} R=\left(x_{1}, \ldots, x_{i}\right) R$. The ideal $\left(x_{1}, \ldots, x_{n}\right) R$ need not be proper. Such a sequence is called permutable if $x_{\sigma(1)}, \ldots, x_{\sigma(n)}$ is a possibly improper regular sequence for every permutation $\sigma$ of $\{1, \ldots, n\}$.)

([EHo] deals only with proper $R$-sequences. The fact that one may use the same formal procedures for computing results of operations on monomial ideals in the improper case can be deduced from the proper case. The issues are local, and so one can assume that part of the sequence is a proper permutable sequence and that the other elements are units. The verification is then quite easy.)

We note that the rules for computing the sum and product of two ideals are valid for arbitrary ideals. Monomial generation is not required. However, the 
rules given for calculating intersections are rarely valid, and the rule for colons is never valid unless one is working with a permutable possibly improper regular sequence (the rule for colons implies the condition that $\left(x_{1}, \ldots, x_{i}\right) R:{ }_{R} x_{i+1} R$ $=\left(x_{1}, \ldots, x_{i}\right) R$, which, in turn, defines such sequences).

If $x_{1}, \ldots, x_{n}$ are elements of $R$ and $\Lambda$ is any ring which maps to $R$, e.g., the prime ring of $R$, then we can always map $A=\Lambda\left[X_{1}, \ldots, X_{n}\right] \rightarrow R$ by mapping $X_{i}$ to $x_{i}$ for every $i$. Every ideal generated by monomials in the $x_{i}$ in $R$ then has the form $I R$, where $I$ is generated by monomials in the $X_{i}$ in $A$. We can then express the result of [EHo], that operations on monomial ideals constructed from permutable regular sequences can be calculated as though the $R$-sequence consisted of indeterminates (generalized to allow possibly improper regular sequences) as follows:

(7.4) Proposition. Let \# denote any of the four binary operations on ideals ,$+ \times,:$, and $\cap$. Let $x_{1}, \ldots, x_{n}$ be a permutable possibly improper regular sequence in $R$. Let $I, J$ be monomial ideals in $A$ (where $A$ is defined as in the paragraph just above). Then $I R \# J R=(I \# J) R$.

In order to prove our main results below, we need two corollaries of this result.

(7.5) Proposition. With notation as in Proposition (7.4), suppose that $R$ has positive prime characteristic $p$. Then for all $q=p^{e}$, and for all monomial ideals $I, J \subseteq A,(I R)^{[q]} \#(J R)^{[q]}=(I \# J)^{[q]} R$.

Proof. $(I R)^{[q]},(J R)^{[q]}$ are the same as $I^{[q]} R, J^{[q]} R$. The result follows readily from the rules given for calculating with monomial ideals in any permutable $R$-sequence.

(7.6) Proposition. Let $S$ be a Noetherian $B$-algebra, where

$$
B=\Lambda\left[X_{1}, \ldots, X_{n}, Z_{1}, \ldots, Z_{d}\right]
$$

is a polynomial over an arbitrary ring $\Lambda$. Let $x_{i}$ (resp. $z_{j}$ ) denote the image of $X_{i}$ (resp. $Z_{j}$ ) in $S$. Suppose that for every subset $T$ of $\{1, \ldots, n\}$ with $t$ elements, the depth of $S$ on the ideal generated by the $z_{j}$ and the $x_{i}$ for $i \in T$ is at least $d+t$. Let $I, J$ be ideals of $B$ generated by monomials in the $X$ 's and $Z$ 's and containing $Z_{1}, \ldots, Z_{d}$. Let \# denote either,$+ \times,:$, or $\cap$. Then IS\#JS = $(I \# J) S$.

Before giving the proof, we note that the ring $\Lambda$ is of no importance here. Since $I, J$ are monomial ideals in indeterminates, so is $I \# J$, and it is computed in a formal way from these monomials. The choice of $\Lambda$ will not affect the meaning of $(I \# J) S$. Thus, given the $x_{i}$ and $z_{j}$ we may always set up a homomorphism as described by taking $\Lambda$ to be either the prime ring in $S$, a subfield of $S$ (if there is a subfield), or $S$ itself, and then sending the indeterminate $X_{i}$ to $x_{i}$ for every $i$ (respectively, $Z_{j}$ to $z_{j}$ for every $j$ ).

Proof of Proposition (7.6). If \# is + or $\times$, there is nothing to prove, and so we may assume that \# is : or $\cap$. The result is local on $S$, and so we may assume that $S$ is local. If the maximal ideal $m$ does not contain all the $z_{j}$, then both 
ideals are the unit ideal, and we are done. Hence, we assume that $\left(z_{j}\right)_{j} S \subseteq m$. We next observe that it is sufficient to check the result working modulo the ideal generated by the $z_{j}$. It is therefore sufficient to prove the result in the case $d=0$. But the hypothesis implies that the $x_{i}$ form a permutable possibly improper regular sequence, and the result now follows from Proposition (7.4).

(7.7) Definition. We shall say that the $x_{1}, \ldots, x_{n} \in R$ are permutable parameters if every subset of $i$ elements generates an ideal of height at least $i$ (i.e., either an ideal of height precisely $i$ or else the unit ideal). This is equivalent to saying that every subset is a set of parameters.

(7.8) Remarks. Permutable parameters retain this property after localization but not necessarily after module-finite extension. Represent

$$
K[[X, Y, Z]] /(X Y, X Z)=K[[x, y, z]]
$$

as a module-finite extension of $K[[y, x-z]]$. This is possible since $y, x-z$ is a system of parameters. Then $y, x-z$ are permutable parameters in the regular ring $K[[y, x-z]]$ but not in its module-finite extension $K[[x, y, z]]$, since $y$ is in a minimal prime. This also illustrates the point that in a local ring $(R, m)$ parameters in $m$ are not automatically permutable. If there are minimal primes of different coheights, there are elements that are part of a system of parameters that generate ideals of height 0 . However, in a local ring that is an equidimensional image of a Cohen-Macaulay local ring, or, more generally, in a local ring whose completion is equidimensional (i.e., that is "quasi-unmixed" in the terminology of $[\mathrm{N}]$ ) parameters in the maximal ideal are automatically permutable. In a C-M ring, $x_{1}, \ldots, x_{n}$ are permutable parameters iff they form a permutable possibly improper regular sequence. However, even in the best rings, parameters may fail to be permutable, either because some subset generates the unit ideal while other subsets are badly behaved, or for more subtle reasons. For example, $x, y(1-x), z(1-x)$ is an $R$-sequence in $K[x, y, z]$ which is not permutable, and these elements are also nonpermutable parameters.

We also note that $x_{1}, \ldots, x_{n}$ are permutable parameters iff for $0 \leq i \leq$ $n-1, x_{i+1}$ is not in any minimal prime of an ideal generated by a subset of $\left\{x_{1}, \ldots, x_{i}\right\}$.

We are now ready for one of the main results of this section. At this point, we impose again the condition that all rings be of characteristic $p$.

(7.9) Theorem. Let $R$ be a locally equidimensional Noetherian ring of characteristic $p$ that is a homomorphic image of a Cohen-Macaulay ring. Let $x_{1}, \ldots, x_{n}$ be permutable parameters in $R$, and map $A=\Lambda\left[X_{1}, \ldots, X_{n}\right]$ to $R$ by sending $X_{i}$ to $x_{i}$, where $\Lambda=\mathrm{Z} / p \mathrm{Z}$. Let $I, J$ be monomial ideals in the $X_{i}$ in $A$, and let \# be any of the operations,$+ \times,:$, and $\cap$. Then

$$
(I \# J) R \subseteq I R \# J R \subseteq((I \# J) R)^{*} \text {. }
$$

In fact, there is a single element $c \in R^{\circ}$ and an integer $q^{\prime}$ such that for all $q \geq q^{\prime}, c(I R \# J R)^{[q]} \subseteq((I \# J) R)^{[q]}$.

Proof. If \# is + or $\times$, then $(I \# J) R=I R \# J R$ for any ideals $I, J$ of $A$ for an arbitrary homomorphism $A \rightarrow R$ of commutative rings. Henceforth, we 
assume that \# is : or $\cap$. The first inclusion is trivial, and we focus on proving $I R \# J R \subseteq((I \# J) R)^{*}$, the interesting part of the theorem. This will follow if we establish the final statement. The result for $R$ follows from the result for $R_{\text {red }}$. (If $c^{\prime}, q^{\prime}$ work for $R_{\text {red }}=R / N, q^{\prime \prime}$ is such that $N^{\left[q^{\prime \prime}\right]}=0$, and $c$ is a lifting of $c^{\prime}$ to $R^{\circ}$, then for every element $u \in I R \# J R$ in $R$, we obtain that $c u^{q} \in((I R+N) \#(J R+N))^{[q]}+N$ for all $q \geq q^{\prime}$, and raising to $q^{\prime \prime}$ powers yields $c^{q^{\prime \prime}} u^{q q^{\prime \prime}} \in(I R \# J R)^{\left[q q^{\prime \prime}\right]}$ for all $q \geq q^{\prime}$, whence $c^{q^{\prime \prime}} u^{q} \in(I R \# J R)^{[q]}$ for all $q \geq q^{\prime} q^{\prime \prime}$.) Henceforth, we assume that $R$ is reduced. If $R$ is a product ring, it suffices to prove the result in each factor separately. Hence, we may assume that $\operatorname{Spec}(R)$ is connected. Write $R$ as $S / Q$, where $S$ is C-M and $Q$ is radical. We claim that the minimal primes $Q_{i}$ of $Q$ in $S$ (which correspond to the minimal primes of $R$ ) all have the same height. To see this, let height $Q_{1}=d$, let $Q^{\prime}$ be the intersection of those $Q_{i}$ that are of height $d$, and let $Q^{\prime \prime}$ be the intersection of those that are not. Then $Q^{\prime} R \cap Q^{\prime \prime} R=0$, and since $\operatorname{Spec}(R)$ is connected, we cannot have $Q^{\prime} R+Q^{\prime \prime} R=R$. It follows that some maximal ideal $m$ of $S$ contains $Q^{\prime}+Q^{\prime \prime}$, and then $m$ contsins both $Q_{i}$ of height $d$ and $Q_{j}$ of height $\neq d$. Since $S_{m}$ is a C-M local ring, it follows that $R_{m}$ is not equidimensional, a contradiction.

Let $W=S-\bigcup_{i} Q_{i}$. Then $W^{-1} S$ is a semilocal ring in which all the maximal ideals have height $d$. We shall show that there are elements $z_{1}, \ldots, z_{d}$ in $Q$ and liftings $y_{1}, \ldots, y_{n}$ of $x_{1}, \ldots, x_{n}$ to $S$ such that the height of the ideal in $S$ generated by $z_{1}, \ldots, z_{d}$ and any subset consisting of $t$ of the $y_{i}, 0 \leq t \leq n$, has height at least $d+t$. This is Lemma (7.10) below. Assuming this, we see that the $z$ 's form a s.o.p. in the local ring of $S$ at each minimal prime of $Q$ (take $t=0$ ). It follows that $Q W^{-1} S$ is $\operatorname{Rad}\left(z_{1}, \ldots, z_{d}\right) W^{-1} S$, and hence we can pick $c^{\prime} \in W$ and a fixed power $q^{\prime}$ of $p$ such that $c^{\prime} Q^{q^{\prime}} \subseteq\left(z_{1}, \ldots, z_{d}\right) S$. We shall show that the image $c$ of $c^{\prime}$ in $R$ and $q^{\prime}$ satisfy our requirements.

Let $B=A\left[Z_{1}, \ldots, Z_{d}\right]$, and map $B \rightarrow S$ sending $X_{i}$ to $y_{i}$ for each $i$ and $Z_{j}$ to $z_{j}$ for each $j$. The induced map to $R$ agrees with the original map on $A$ and sends all the $Z_{j}$ to 0 . Now suppose that $s \in S$ and its image $r$ in $R$ is in $I R \# J R$. Let $I^{\prime}=I B+\left(Z_{j}\right)_{j} B$ and $J^{\prime}=J B+\left(Z_{j}\right)_{j} B$. It is easy to see that $I^{\prime} \# J^{\prime}=(I \# J) B+\left(Z_{j}\right) B$. We have then that $I^{\prime} S \# J^{\prime} S=\left(I^{\prime} \# J^{\prime}\right) S$, by Proposition (7.6). It follows that $s \in(I S+Q) \#(J S+Q)$, and so for all $q \geq q^{\prime}$, we have (recalling that $c^{\prime} Q^{q^{\prime}} \subseteq\left(z_{j}\right)_{j} S$ )

$$
\begin{aligned}
c^{\prime} s^{q} \in\left(I^{[q]} S+\left(z_{j}\right)_{j} S\right) \#\left(J^{[q]} S+\left(z_{j}\right)_{j} S\right) \\
=\left(I^{[q]} \# J^{[q]}+\left(z_{j}\right)_{j}\right) S \\
=\left((I \# J)^{[q]}+\left(z_{j}\right)\right) S .
\end{aligned}
$$

Taking images in $R$ yields that $c r^{q} \in(I \# J)^{[q]} R=((I \# J) R)^{[q]}$ for all $q \geq q^{\prime}$, and the result therefore follows from Lemma (7.10) below. 
(7.10) Lemma. Let $S$ be any catenary Noetherian ring, e.g., a Cohen-Macaulay ring, and let $Q$ be a proper ideal of $S$ of height $d$. Let $R=S / Q$, and let $x_{i}$, $1 \leq i \leq n$, be elements of $R$ that are permutable parameters. Then there exist elements $z_{1}, \ldots, z_{d} \in Q$ and liftings $y_{i}$ of the $x_{i}$ to $S$ such that for any set $T \subseteq\{1, \ldots, n\}$ with $t$ elements, $0 \leq t \leq n$, the ideal generated by the $z_{j}$ and those $y_{i}$ with $i \in T$ has height at least $d+t$.

Proof. First lift the $x_{i}$ to elements $y_{i}$ of $S$ inductively in such a way that for every $i, 0 \leq i \leq n-1, y_{i+1}$ is not in any minimal prime of an ideal generated by a subset of its predecessors. (Let $y$ denote any element of $S$ that lifts $x_{i+1}$. If there were no such lifting, then, by standard prime avoidance techniques, for example Theorem 124 on p. 90 of [Kap], $R y+Q$ is contained in a minimal prime $P$ of $\left(y_{1}, \ldots, y_{m}\right) S$, where $m \leq i$. But then, since height $Q=d$, since the images of the elements $y_{1}, \ldots, y_{m}, y$ in $R=S / Q$, are $x_{1}, \ldots, x_{m}, x_{i+1}$, and since $S$ is catenary, we have that $P$ has height at least $d+m+1$. On the other hand, height $P \leq m$, a contradiction.) Second, choose $z_{1}, \ldots, z_{d} \in Q$ inductively such that for every $j, 0 \leq j \leq d-1$, $z_{j+1}$ is not in any minimal prime of the ideal generated by $\left(z_{1}, \ldots, z_{j}\right) S$ and a subset of the $y_{j}$. (If this were impossible for a certain $t$ element subset of the $y_{j}$, say $\left\{y_{1}, \ldots, y_{t}\right\}$, it would follow that there exists a minimal prime $P$ of $\left(z_{1}, \ldots, z_{j}\right) S+\left(y_{1}, \ldots, y_{t}\right)$ that contains $Q$. Working in $S_{P}$, we have that the height of the ideal $Q+\left(y_{1}, \ldots, y_{t}\right)$ is at least $d+t$, since the ring is catenary, height $Q=d$, and the $y_{i}$ reduce to the parameters $x_{i}$ modulo $Q$. On the other hand, the height of $P$ is $\leq j+t$, which contradicts the assumption $j \leq d-1$.) It follows by induction on $j$ that the height of the ideal generated by $\left(z_{1}, \ldots, z_{j}\right) S$ and any $t$ element subset of the $y_{i}$ is at least $t+j, 0 \leq j \leq d$. In particular, we know this for $j=d$, which proves the lemma.

The next theorem will enable us to prove results for iterated operations. We give it in a rather general form that can be applied in a number of different contexts.

Before giving the statement, we need to consider certain special classes of ideal-valued functions.

(7.11) Discussion. Let $U_{1}, \ldots, U_{k}$ denote variable ideals. We consider idealvalued functions of these variables that can be constructed recursively from the functions $U_{i}$ (technically, this is the $i$ th projection), $U_{i}+U_{j}, U_{i} U_{j}, U_{i} \cap U_{j}$, and $U_{i}: U_{j}$ by allowing the substitution of functions already constructed for any of the variables $U_{i}, U_{j}$ in the functions listed above, subject to the restriction that one may substitute only a function for the numerator $U_{i}$ of $U_{i}: U_{j}$, and not for the denominator $U_{j}$. We call the functions that can be constructed in this way permissible. For example, $\left(U_{1}: U_{2}\right): U_{3}$ is permissible as is

$$
\left(\left(U_{1} U_{2}+\left(\left(U_{3}: U_{2}\right): U_{1}\right)\right) \cap U_{4}\right) \cap\left(\left(U_{5}+\left(U_{6}: U_{3}\right)\right): U_{2}\right) \text {. }
$$

However, $U_{1}:\left(U_{2}: U_{3}\right)$ and $U_{1}:\left(U_{2} \cap U_{3}\right)$ are not permissible. We note that while $U_{1}:\left(U_{2}+U_{3}\right)$ is not permissible a priori, it can be rewritten as $\left(U_{1}: U_{2}\right) \cap$ $\left(U_{1}: U_{3}\right)$, which is permissible. Likewise, $U_{1}:\left(U_{2} U_{3}\right)$ is not permissible a priori, but can be rewritten as $\left(U_{1}: U_{3}\right): U_{3}$, which is. 
We shall call an ideal-valued function of ideals almost permissible if it can be constructed recursively from the functions $U_{i}, U_{i}+U_{j}, U_{i} \cap U_{j}, U_{i}: U_{j}$ by substitution of functions already constructed from the variables, subject to the restriction that a function substituted for $U_{j}$ in $U_{i}: U_{j}$ must be permissible. For example, $U_{1}:\left(U_{2}: U_{3}\right)$ is almost permissible, as is

$$
\left(\left(\left(U_{1}: U_{5}\right)+\left(U_{2} \cap U_{3}\right)\right):\left(U_{1} U_{4}+\left(U_{7} \cap\left(U_{2}: U_{5}\right)\right)\right)\right)+\left(U_{5}:\left(U_{1}: U_{3}\right)\right) \text {, }
$$

but $U_{1}:\left(U_{2}:\left(U_{3}: U_{4}\right)\right)$ is not almost permissible.

Given a homomorphism $A \rightarrow R$ and an ideal $I$ of $A$, where $A, R$ are Noetherian of characteristic $p$, then we shall say that $I^{\prime} \subseteq R$ is trapped over $I$ if $I R \subseteq I^{\prime} \subseteq(I R)^{*}$.

The following rather lengthy theorem will enable us to deduce results on iterated operation in numerous situations.

(7.12) Theorem (key lemma for iterated operations). Let $\mathscr{I}$ be a family of ideals of a Noetherian ring $A$ of characteristic $p$ such that if $I, J$ belong to $\mathcal{I}$, then so do $I+J, I J, I: J, I \cap J$, and $I^{[q]}$ for all $q=p^{e}$. Let $h: A \rightarrow R$ be a ring homomorphism.

Suppose that the following condition holds.

$$
\begin{aligned}
& \text { For all } I, J \in \mathcal{I},(I R)^{*} \cap(J R)^{*} \subseteq((I \cap J) R)^{*} \text { and }(I R)^{*}: J R \\
& \subseteq((I: J) R)^{*} .
\end{aligned}
$$

Then for all $I, J \in \mathscr{I},(I R)^{*} \cap(J R)^{*}=((I \cap J) R)^{*}$ and $(I R)^{*}: J R=$ $((I: J) R)^{*}$. What is more, if $I, J \in \mathscr{I}, I R \subseteq I^{\prime} \subseteq(I R)^{*}$, and $J R \subseteq$ $J^{\prime} \subseteq(J R)^{*}$ (i.e., $I^{\prime}$ is trapped over $I$ and $J^{\prime}$ is trapped over $J$ ), we have the following.

(a) $(I \# J) R \subseteq I^{\prime} \# J^{\prime} \subseteq((I \# J) R)^{*}$ if \# is + , $\times$, or $\cap$.

(b) $\left(I:{ }_{A} J\right) R \subseteq I^{\prime}:{ }_{R} J R \subseteq\left(\left(I:{ }_{A} J\right) R\right)^{*}$ (which implies that $I^{\prime}:{ }_{R} J^{\prime} \subseteq$ $\left.\left(\left(I:{ }_{A} J\right) R\right)^{*}\right)$.

It follows that if $\mathscr{A}$ is a permissible (respectively, an almost permissible) idealvalued function of $k$ variable ideals and $I_{1}, \ldots, I_{k} \in \mathscr{I}, \underline{I}=\left(I_{1}, \ldots, I_{k}\right)$, and $\underline{I} R$ denotes $\left(I_{1} R, \ldots, I_{k} R\right)$, then $\mathscr{A}(\underline{I} R)$ is trapped over $\mathscr{A}(\underline{I})$, i.e., $\mathscr{A}(\underline{I}) R \subseteq$ $\mathscr{A}(\underline{I} R) \subseteq(\mathscr{A}(\underline{I}) R)^{*}$ (respectively, $\left.\mathscr{A}(\underline{I} R) \subseteq(\mathscr{A}(\underline{I}) R)^{*}\right)$.

If every ideal in $\mathscr{I}$ is a sum of principal ideals aA $\in \mathscr{I}$ such that $a$ is a nonzerodivisor on $R$, then for (**) to hold, it suffices that for all $I, J \in \mathscr{I}$, $(I R)^{*} \cap(J R)^{*} \subseteq((I \cap J) R)^{*}$.

Finally, we note ihat if $A$ is regular, a sufficient condition for the inclusion $(I R)^{*} \cap(J R)^{*} \subseteq((I \cap J) R)^{*}$ (respectively, $\left.(I R)^{*}: J R \subseteq((I: J) R)^{*}\right)$ to hold for all $I, J \in \mathscr{I}$ is that there exist $c \in R^{\circ}$ and an integer $q^{\prime}$ such that $c(I R \cap J R)^{\left[q^{\prime}\right]} \subseteq((I \cap J) R)^{\left[q^{\prime}\right]}$ (respectively, $\left.c\left(I^{*} R: J R\right)^{\left[q^{\prime}\right]} \subseteq((I: J) R)^{\left[q^{\prime}\right]}\right)$ for all $I, J \in \mathcal{I}$. Thus, if one has $c, q^{\prime}$ that satisfy the indicated conditions for both $\cap$ and : , then (**) holds and the statements above on behavior of permissible and almost permissible functions are valid.

Proof. The statement that the inclusions in condition (**) imply equality is trivial. The opposite inclusions follow from the facts that $(I R)^{*} \cap(J R)^{*}$ and $(I R)^{*}: J R$ are tightly closed; see Proposition (4.1). The fact that (a) and (b) 
hold is then obvious. There is no problem at all when \# is + or $x$. (a) and (b) can be paraphrased as follows. If $I^{\prime}, J^{\prime}$ are trapped over $I, J \in \mathcal{I}$, respectively, then $I^{\prime} \# J^{\prime}$ is trapped over $I \# J$, subject to the restriction that if \# is : then $J^{\prime}$ is equal to $J R$ (and not just trapped over $J$ ). The result on permissible functions is then immediate from (a), (b) by induction on the number of substitutions needed in the recursive construction of the function. The result on almost permissible functions is also immediate by the same line of argument, but now making use of the remark that when $I^{\prime}, J^{\prime}$ are trapped over $I, J$, then $I^{\prime}: J^{\prime} \subseteq((I: J) R)^{*}$, although $I^{\prime}: J^{\prime}$ need no longer contain $(I: J) R$.

It remains only to establish the sufficiency of the criteria for $(* *)$ to hold given in the last two paragraphs of the statement of the theorem. First, suppose that every ideal of $\mathscr{I}$ is a sum of principal ideals of $\mathscr{I}$ generated by nonzerodivisors on $R$. Suppose $I, J \in \mathscr{I}$ are given and $J=\left(a_{1}, \ldots, a_{m}\right) A$, where each $a_{i}$ is a nonzerodivisor on $R$ and each $a_{i} A \in \mathscr{I}$. Then $(I R)^{*}: J R=$ $\bigcap_{i}(I R)^{*}:\left(a_{i} R\right)$. If $a=a_{i}$ and $z \in\left(I R^{*}\right):(a R)$ and we have that $a z \in$ $(I R)^{*} \cap a R \subseteq((I \cap a A) R)^{*}=(a(I: a A) R)^{*}$, which implies that $z \in((I: a A) R)^{*}$ by Lemma (7.13) below. But then

$$
(I R)^{*}: J R \subseteq \bigcap_{i}\left(\left(I: a_{i} A\right) R\right)^{*} \subseteq\left(\left(\bigcap_{i}\left(I: a_{i} A\right)\right) R\right)^{*}
$$

(by repeated use of the property for intersections) $=((I: J) R)^{*}$, as required.

The criterion in the last paragraph of the statement of the theorem is perhaps the most interesting part. We must show under the hypothesis that $c, q^{\prime}$ as specified exist that if $u \in(I R)^{*} \cap(J R)^{*}$ (respectively, $\left.(I R)^{*}: J R\right)$, then $u \in$ $((I \cap J) R)^{*}$ (respectively, $\left.((I: J) R)^{*}\right)$. We choose $c^{\prime} \in R^{\circ}$ and $q^{\prime \prime}$ such that for all $q \geq q^{\prime \prime}, c^{\prime} u^{q} \in(I R)^{[q]} \#(J R)^{[q]}=I^{[q]} R \# J^{[q]} R$. Since $I^{[q]}, J^{[q]}$ are again in $\mathscr{I}$, it follows that for all $q \geq q^{\prime \prime}$ we have $c\left(c^{\prime} u^{q}\right)^{q^{\prime}} \in\left(\left(I^{[q]} \# J^{[q]}\right) R\right)^{\left[q^{\prime}\right]}=$ $\left((I \# J)^{[q]} R\right)^{\left[q^{\prime}\right]}$ (since $A$ is regular) $=((I \# J) R)^{\left[q q^{\prime}\right]}$, which implies that for all $q \geq q^{\prime} q^{\prime \prime},\left(c c^{\prime q^{\prime}}\right) u^{q} \in((I \# J) R)^{[q]}$.

This completes the argument, once we have established Lemma (7.13) below.

(7.13) Lemma. Let $a$ be a parameter of $R$ and $J$ any ideal. If $a z \in(a J)^{*}$, then $z \in J^{*}$.

Proof. We can pass to $R_{\text {red }}$ and so assume that $a$ is a nonzerodivisor. We can choose $c \in R^{\circ}$ such that for all large $q$ we have $c(a z)^{q} \in(a J)^{[q]}=a^{q} J^{[q]}$. Since $a$ is not a zerodivisor, this implies that for all large $q$ we have that $c z^{q} \in J^{[q]}$.

(7.14) Remarks. (a) In order to prove that $(I R)^{*} \cap(J R)^{*}=((I \cap J) R)^{*} \quad(\mathrm{re}-$ spectively, that $\left.(I R)^{*}: J R=((I: J) R)^{*}\right)$ from the condition in the last paragraph of the statement of Theorem (7.12), we did not really need to work with such a large family $\mathscr{I}$. What we really needed in the argument is simply the existence of a fixed element $c^{\prime} \in R^{\circ}$ and a fixed integer $q^{\prime}$ such that for all $q$, 
$c^{\prime}\left(\left(I^{[q]} R \cap J^{[q]}\right) R\right)^{\left[q^{\prime}\right]} \subseteq\left(\left(I^{[q]} \cap J^{[q]}\right) R\right)^{\left[q^{\prime}\right]}$ (respectively, $c^{\prime}\left(\left(I^{[q]} R: J^{[q]} R\right)^{\left[q^{\prime}\right]}\right) \subseteq$ $\left.\left(\left(I^{[q]}: J^{[q]}\right) R\right)^{\left[q^{\prime}\right]}\right)$. The result is stated in terms of a family so that it can be applied to iterated operations.

(b) The condition that $A$ be regular in the last paragraph of Theorem (7.12) is not entirely essential. All we really need is that the Frobenius power operation (sending $I$ to $I^{[q]}$ ) commute with : and $\cap$, which might be guaranteed by other means, e.g., by assuming that various ideals have finite projective dimension.

(c) Suppose that $I^{*} R \subseteq(I R)^{*}$ for each $I \in \mathscr{I}$, which is automatic if $A$ is regular or $A^{\circ}$ maps into $R^{\circ}$. Then the conclusion of Theorem (7.12) remains valid if we enlarge the classes of permissible and almost permissible functions by allowing the substitution of $U_{i}^{*}$ for $U_{i}$. The point is that if an ideal is trapped over $I$, its tight closure is trapped over $I^{*}$, and even over $I$, since $I R \subseteq I^{*} R \subseteq\left(I^{*} R\right)^{*}=(I R)^{*}$.

(d) We may likewise enlarge both classes of functions by permitting the substitution $U_{i}^{[q]}$ for $U_{i}$, for if an ideal is trapped over $I$, this is preserved when we apply ${ }^{[q]}$. The key point is that $\left((I R)^{*}\right)^{\left[q^{\prime}\right]} \subseteq\left(I^{\left[q^{\prime}\right]} R\right)^{*}$. (For suppose $c \in R^{\circ}$ and $c y^{q} \in I^{[q]} R$ for $q \gg 0$, i.e., $y \in(I R)^{*}$. Then $c^{q^{\prime}}\left(y^{q^{\prime}}\right)^{q} \in\left(I^{\left[q^{\prime}\right]} R\right)^{[q]}$ for $q \gg 0$, as required.)

We are now ready to state our main result on iterated operations, which gives several contexts in which the result of performing iterated operations is in the tight closure of the expected answer. The first of these, Theorem (7.15)(a) is the culmination of the work we did earlier on permutable parameters in locally equidimensional homomorphic images of C-M rings.

(7.15) Theorem (main theorem on iterated operations). Let $A \rightarrow R$ be a homomorphism of Noetherian rings of characteristic $p$, and let $\mathscr{I}$ be a family of ideals of $A$. Then in each of the situations (a), (b), (c), (d), (e), and (f) listed below, for every permissible (respectively, almost permissible) ideal-valued function $\mathscr{A}$ of $k$ variable ideals $I_{1}, \ldots, I_{k} \in \mathscr{I}$ if $\underline{I}$ denotes $\left(I_{1}, \ldots, I_{k}\right)$, and $\underline{I} R=\left(I_{1} R, \ldots, I_{k} R\right)$, then $\mathscr{A}(\underline{I} R)$ is trapped over $\mathscr{A}(\underline{I})$, i.e., $\mathscr{A}(\underline{I}) R \subseteq$ $\mathscr{A}(\underline{I} R) \subseteq(\mathscr{A}(\underline{I}) R)^{*}$. (respectively, $\left.\mathscr{A}(\underline{I} R) \subseteq(\mathscr{A}(\underline{I}) R)^{*}\right)$.

(a) $R$ is a locally equidimensional homomorphic image of a Cohen-Macaulay ring, $x_{1}, \ldots, x_{n}$ are permutable parameters, $\Lambda=\mathbf{Z} / p \mathbf{Z}, A=\Lambda\left[X_{1}, \ldots, X_{n}\right]$, a polynomial ring, mapping to $R$ so that the image of $X_{i}$ is $x_{i}$, and $\mathscr{I}$ is the family of monomial ideals in the $X_{i}$ in $A$.

(b) $R$ is a module-finite extension of a regular domain $A$ such that $R$ is torsion-free as an A-module or, more generally, such that $R$ is locally equidimensional, and $\mathscr{I}$ is the family of all ideals of $A$.

(c) $A$ is a regular ring such that every residue class field is perfect, $R$ is locally formally equidimensional and has a completely stable $q$ '-weak test element, $h: A \rightarrow R$ is such that every maximal ideal of $R$ contracts to a maximal ideal of $A$ and such that for all maximal ideals $m$ of $A$, height $m R \geq$ height $m$, and $\mathcal{I}$ is the family of all ideals of $A$.

(d) $h: A \rightarrow R$ is a local homomorphism of complete local rings such that $(A, P)$ is regular, $R$ is equidimensional, $A$ has a coefficient field $K$ that can be extended to a coefficient field of $R$, height $P R=\operatorname{dim} A$ (so that regular 
parameters for $A$ are part of a system of parameters in $R$ ), and $\mathscr{I}$ is the family of all ideals of $A$.

(e) $A$ is a regular ring finitely generated over a perfect field $K, R$ is a locally equidimensional ring finitely generated as an A-algebra such that for all maximal ideals $m$ of $A$, height $m R \geq$ height $m$, and $\mathcal{I}$ is the family of all ideals of $A$.

(f) $A$ is a polynomial ring in $d$ variables over a perfect field $K, R$ is $\mathbf{N}$ graded, locally formally equidimensional and has a completely stable $q^{\prime}$-weak test element, $h: A \rightarrow R$ is a graded homomorphism such that every maximal ideal of $R$ contracts to a maximal ideal of $A$ and such that for the graded maximal ideal $m$ of $A$, height $m R \geq d$, and $\mathscr{I}$ is the family of all homogeneous ideals of $A$.

Before giving the proof of this result, we make some remarks and then prove a result that shows that nilpotents may, in essence, be ignored in this context.

(7.16) Remarks and examples. The reason that the colon function $U: V$ requires special treatment is that as the denominator $V$ gets larger the value of $U: V$ gets smaller. In the case of all the other ideal-valued functions that we are considering (as well as for the numerator $U$ of $U: V$ ), enlarging the value of the variable ideal enlarges the value of the function. For this reason, $I^{\prime}: J^{\prime}$ need not be trapped over $I: J$ when $J^{\prime}$ is strictly larger than $J$. This is what forces us to make the rather technical definitions of permissible and almost permissible function.

Specifically, let $(R, m)$ be a two-dimensional equidimensional local ring of characteristic $p$ that is not C-M and let $x, y$ be a s.o.p. $\left(K\left[\left[u^{4}, u^{3} v, v u^{3}, v^{4}\right]\right]\right.$ $\subseteq K[[u, v]]$ is one completely specific example. We may take $x=u^{4}, y=v^{4}$.) Let $A=\mathbf{Z}_{p}[X, Y]$. Since $x, y$ is not a regular sequence, $x R: y R$ is strictly larger than $x R$, and so $x R:(x R: y R) \subseteq m$, and is not trapped over $X A:(X A: Y A)=X A: X A=A$, although it is contained in $A R=$ $R$. This is the kind of behavior we expect from an almost permissible function (i.e., $\left.U_{1}:\left(U_{2}: U_{3}\right)\right)$ that is not permissible. Since $m$ is nilpotent modulo $(x, y) R$, we can choose $t$ such that $(x R:(x R: y R))^{t} \subseteq(x, y) R$, and then $(x, y) R:(x R:(x R: y R))^{t}=R$ is not even contained in the tight closure of the expected answer, which is $\left((X, Y) A:(X A:(X A: Y A))^{t}\right) R=$ $\left((X, Y) A: A^{t}\right) R=(X, Y) R=(x, y) R$ (note that $t$ th powers are obtainable as iterated products). The function involved, $U_{1}:\left(U_{2}:\left(U_{3}, U_{4}\right)\right)^{t}$, is not almost permissible, since the denominator is not permissible.

(7.17) Proposition. Let $h, A, R$, and $\mathscr{I}$ be as in the first paragraph of Theorem (7.12). Then condition (**) holds iff it holds for $h^{\prime}, A, R_{\mathrm{red}}$, and $\mathscr{I}$, where $h^{\prime}$ is the composition of $h$ with the canonical surjection $R \rightarrow R_{\mathrm{red}}$.

Proof. Let $N$ be the nilradical of $R$ and suppose that $N^{\left[q^{\prime \prime}\right]}=0$. First assume that $(* *)$ holds in the original situation, and let $I, J \in \mathscr{I}$. Let $u \in R$ be such that $u+N \in\left(I R_{\text {red }}\right)^{*} \cap\left(J R_{\text {red }}\right)^{*}$ (respectively, $\left.\left(I R_{\text {red }}\right)^{*}: J R_{\text {red }}\right)$. Then there is a $c \in R^{\circ}$ and $q^{\prime}$ such that for all $q \geq q^{\prime},(c+N)(u+N)^{q} \in\left(I^{[q]} R+N\right) \cap\left(J^{[q]} R+N\right)$ (respectively, $\left.(c+N)(u+N)^{q} J^{[q]} R \subseteq I^{[q]} R+N\right)$. Raising both sides to the 
$q^{\prime \prime}$ power, we find that for all $q \geq q^{\prime} q^{\prime \prime}, c^{q^{\prime \prime}} u^{q} \in I^{[q]} R \cap J^{[q]} R$ (respectively, $\left.c^{q^{\prime \prime}} u^{q} J^{[q]} R \subseteq I^{[q]} R\right)$, whence $u \in(I R)^{*} \cap(J R)^{*} \subseteq((I \cap J) R)^{*}$ (respectively, $\left.u \in(I R)^{*}: J R \subseteq\left(\left(I:{ }_{A} J\right) R\right)^{*}\right)$, and so $u+N \in\left((I \cap J) R_{\text {red }}\right)^{*}$ (respectively, $\left.u+N \in\left(\left(I:{ }_{A} J\right) R_{\text {red }}\right)^{*}\right)$, as required.

Now assume that $(* *)$ holds after we pass to $R_{\text {red }}$, and let $u \in(I R)^{*} \cap(J R)^{*}$ (respectively, $\left.(I R)^{*}: J R\right)$. Evidently, $u+N$ is in $\left(I R_{\text {red }}\right)^{*} \cap\left(J R_{\text {red }}\right)^{*}$ (respectively, $\left.\left(I R_{\text {red }}\right)^{*}: J R_{\text {red }}\right)$, and so in $\left((I \cap J) R_{\text {red }}\right)^{*}$ (respectively, $\left.\left((I: J) R_{\text {red }}\right)^{*}\right)$. The conclusion we need is now immediate from Proposition $(4.1)(\mathrm{j})$.

Proof of Theorem 7.16. (a) is immediate from Theorems (7.9) and (7.12).

(b) If $R$ is locally equidimensional, we begin by passing to $R_{\text {red }}$. This is justified by Proposition (7.17). The condition is equivalent to being torsion-free over $A$ once we know that $R$ is reduced. It then suffices to show that there exist $c \in R^{\circ}$ and $q^{\prime}$ such that for all $I, J \subseteq A, c(I R \cap J R)^{\left[q^{\prime}\right]} \subseteq((I \cap J) R)^{\left[q^{\prime}\right]}$, by virtue of the criteria for (**) given in Theorem (7.12). But, precisely as in the proof of Theorem (4.8), we may choose $q^{\prime}=1$ and $c$ to be an element of $A-\{0\}$ such that $c R \subseteq F$, where $F$ denotes a free $A$-submodule of $R$ of maximum rank.

(c) By Theorem (7.12) all we need to check is that if $I, J$ are ideals of $A$, then $(I R)^{*} \cap(J R)^{*} \subseteq((I \cap J) R)^{*}$ and $(I R)^{*}: J R \subseteq((I: J) R)^{*}$. Let $\mathscr{A}=(I \cap J) R$ (respectively, $(I: J) R)$, and suppose $u$ denotes an element of $(I R)^{*} \cap(J R)^{*}$ (respectively, $(I R)^{*}: J R$ ) that is not in the ideal $\mathscr{A}^{*}$ on the right in the respective inclusion that we are trying to prove. Let $c$ be a completely stable $q^{\prime}$-weak test element. Then $c u^{q}$ is not in $\mathscr{A}^{[q]}$ for some choice of $q \geq q^{\prime}$, and this will be preserved after localizing and completing at a certain maximal $m$ of $R$. Let $S$ be the localized completion of $R$, and let $B$ be the completion of the localization of $A$ at the contraction $m^{\prime}$ of $m$. Then since $(I R)^{*},(J R)^{*}$ map into $(I S)^{*},(J S)^{*}$, respectively, we get a new counterexample in which $A, R, I, J$ are replaced by $B, S, I B, J B$. (We are using the fact that the image of $c$ is a $q^{\prime}$-weak test element in $S$.) Note that since $m^{\prime}$ is maximal in $A, B$ is a complete regular local ring with a perfect residue field. Note also that $\operatorname{since} \operatorname{dim} B=$ height $m^{\prime} \leq$ height $m^{\prime} R \leq$ height $m^{\prime} R_{m}=$ height $m^{\prime} S$, we can conclude that if $x_{1}, \ldots, x_{d}$ is a regular system of parameters for $B$, then it is part of a system of parameters for $S$. The height condition forces the map $B \rightarrow S$ to be injective. We have now reduced to the situation of part (d), which is proved below.

(d) Let $L$ be a coefficient field for $R$ extending $K$, and let $x_{1}, \ldots, x_{d}$ generate $P$, so that $A=K\left[\left[x_{1}, \ldots, x_{d}\right]\right]$. Then we can enlarge $x_{1}, \ldots, x_{d}$ to a system of parameters $x_{1}, \ldots, x_{n}$ for $S$ and then $A$ by $A^{\prime}=L\left[\left[x_{1}, \ldots, x_{n}\right]\right]$, since $A^{\prime}$ is faithfully flat over $A$. Changing notation, we may assume that $A=A^{\prime}$, and then $R$ is module-finite over the regular local ring $A$. The result now follows from part (b).

(e) Since $R, A$ are of finite type over $K$, we know that every maximal ideal of $R$ lies over a maximal ideal of $A$. Since $R$ is excellent, its local rings are formally equidimensional, not just equidimensional. Moreover, $R$ has a completely stable $q^{\prime}$-weak test element. Thus, part (c) applies. 
(f) We imitate the proof of part (c). The point is that we can choose the maximal ideal of $R$ at which we are going to localize to be homogeneous. It then lies over a homogeneous maximal ideal of $A$, which must be $m$, and the rest of the argument is the same.

\section{TIGHT CLOSURE FOR SUBMODULES IN CHARACTERISTIC $p$}

In this section, we extend the notion of tight closure for ideals to submodules of a finitely generated module over a Noetherian ring $R$ of characteristic $p$. After giving the basic definitions and exploring some fundamental properties, we extend the notion of test element for ideals to the module case, and develop some results comparing the two notions. We also develop a criterion for one submodule to be in the tight closure of another when their quotient has finite length in terms of conditions on asymptotic growth of lengths as iterations of the Frobenius endomorphism are applied. See Theorem (8.17). In a different direction, we consider the extent to which the tight closure of a torsion-free module embedded in a projective module is independent of the embedding (this is the case for normal rings; see Proposition (8.18)).

We also consider a variant notion (finitistic tight closure) that is useful in situations where modules are not necessarily finitely generated, and a notion of absolute tight closure that gives rise to right derived functors.

Throughout this section, rings are assumed to be of positive prime characteristic $p$ unless otherwise specified.

(8.1) Discussion. We begin by briefly recalling some facts about the PeskineSzpiro functors $F^{e}$, where $e \in \mathbf{N}$. Let $S$ denote $R$ viewed as an $R$-algebra via the $e$ th power of the Frobenius endomorphism. Then $F^{e}$ is simply $S \otimes_{R}$, a covariant functor from $R$-modules to $S$-modules. However, since $S=R$, this is a covariant functor from $R$-modules to themselves. If we apply $F^{e}$ to a map $R^{n} \rightarrow R^{m}$ with matrix $\left(r_{i j}\right)$, we obtain the map between the same two modules with matrix $\left(r_{i j}^{q}\right)$, where $q=p^{e}$. Note that the $R$-module structure on $F^{e}(M)$ is such that $r^{\prime}(r \otimes m)=\left(r^{\prime} r\right) \otimes m$. On the other hand, $r^{\prime} \otimes(r m)=\left(r^{\prime} r^{q}\right) \otimes m$. Also note that $F^{e}(R / I)=R / I^{[q]}$, where $q=p^{e}$.

We observe that there is a canonical map $M \rightarrow F^{e}(M)$ that sends $m$ to $1 \otimes m$. If $q=p^{e}$ and $x \in M$, we shall write $x^{q}$ for the image of $x$ in $F^{e}(M)$. Note that $(x+y)^{q}=x^{q}+y^{q}$ and $(r x)^{q}=r^{q} x^{q}$ with this notation. Note also that if $x=\left(r_{1}, \ldots, r_{n}\right) \in M=R^{n}$, then $x^{q}=\left(r_{1}^{q}, \ldots, r_{n}^{q}\right)$ if we identify $F^{e}(M)$ with $R^{n}$. In particular, this new notation is consistent with our notation for the case $M=R$. If $N \subseteq M$, we shall write $N^{[q]}$ for $\operatorname{Ker}\left(F^{e}(M) \rightarrow F^{e}(M / N)\right)$. By the right exactness of $\otimes, N^{[q]}$ may also be identified with $\operatorname{Im}\left(F^{e}(N) \rightarrow F^{e}(M)\right)$, and this is the same as the $R$-span in $F^{e}(M)$ of the elements $x^{q}$ for $x \in N . N^{[q]}$ depends heavily on what $M$ is (or, more precisely, on what $N \rightarrow M$ is). If we need to indicate this dependence on $M$ in the notation, we shall write $N_{M}^{[q]}$ instead. With these conventions $M_{M}^{[q]}=F^{e}(M)$, and we sometimes write $M^{[q]}$ for $F^{e}(M)$. 
Note that if $M=R$ and $N=I \subseteq R$, then $I_{R}^{[q]}$ in this new notation is identical with what was previously described as $I^{[q]}$.

(8.2) Definition. Let $N \subseteq M$ be modules over a ring $R$ of characteristic $p$. We say that $x \in M$ is in the tight closure $N^{*}$ of $N$ if there exist $c \in R^{\circ}$ and an integer $q^{\prime}$ such that for all $q \geq q^{\prime}, c x^{q} \in N_{M}^{[q]}$. If $N=N^{*}$, we say that $N$ is tightly closed (in $M$ ).

(8.3) Remark. The notation $N^{*}$ is often used for the dual of a module in some sense. Therefore, we shall also use the alternative notation $\mathrm{Cl}(N)$ for the tight closure. Since the tight closure will depend on what $M$ is as well, we shall sometimes indicate the larger module in our notation by writing $N_{M}^{*}$, $\mathrm{Cl}_{M}(N)$, or $\mathrm{Cl}(N, M)$. The problem will not arise significantly in the present manuscript, since we have little or no need to refer to duals.

(8.4) Remark. It is easy to see that $x \in M$ is in the tight closure of $N \subseteq$ $M$ if and only if $x+N$ is in the tight closure of 0 in $M / N$. In fact, $c x^{q} \in N_{M}^{[q]}$ iff $c(x+N)^{[q]} \in 0_{M / N}^{[q]}=0$ in $F^{e}(M / N)=F^{e}(M) / N^{[q]}$, since $c(x+N)^{q}$ is represented by $c x^{q}$. One may always translate questions about whether an element is in the tight closure of a submodule first to the case where the submodule $N$ is 0 , and then, using the reverse trick, to the case where $M$ is free, by mapping a free module $G$ onto $M / N$ and studying the question for $G$ and the inverse image of the submodule 0 .

We have given this definition without restricting $M$ to being finitely generated. Our main interest is certainly in the case of finitely generated modules, but, in the result just below, we keep track of which basic properties hold in general. In the sequel, we make a blanket assumption that given modules are finitely generated, unless otherwise specified. We should point out that later in the section, we shall define the notion of the "absolute" tight closure of a submodule. It is of some interest to apply this to modules that are not finitely generated, for taking the absolute tight closure of the 0 submodule of a module is a left exact functor. Hence, it has right derived functors, and we need to apply the notion to injective modules.

The following proposition summarizes many of the basic properties of tight closure for submodules.

(8.5) Proposition. Let $R$ be a Noetherian ring of characteristic $p$, let $M$ be an $R$-module, let $N, N^{\prime}$, etc. denote submodules of $M$, and let $I$ denote an ideal of $R$.

(a) $N_{M}^{*}$ is a submodule of $M$ containing $N$.

(b) If $N \subseteq N^{\prime} \subseteq M$, then $N_{M}^{*} \subseteq N_{M}^{\prime *}$. The intersection of an arbitrary family of tightly closed submodules of $M$ is tightly closed.

(c) If $\mathrm{Ann}_{R} M / N$ has positive height or if $R$ is reduced, then $x \in N^{*}$ iff there exists $c \in R^{\circ}$ such that $c x^{q} \in N_{M}^{[q]}$ for all $q=p^{e}$. More generally, if $x \in M$ and $\operatorname{Ann}_{R}(x+N)=N:{ }_{R} R x$ has positive height, then $x \in N^{*}$ iff there exists $c \in R^{\circ}$ such that $c x^{q} \in N^{[q]}$ for all $q$.

(d) If $M$ is finitely generated, then for every submodule $N \subseteq M$, there exist $c \in R^{\circ}$ and $q^{\prime}$ such that $c\left(N_{M}^{*}\right)^{[q]} \subseteq N_{M}^{[q]}$ for all $q \geq q^{\prime}$. If $\operatorname{Ann}_{R} M / N$ has 
positive height or if $R$ is reduced, then $c$ can be chosen so that the inclusion holds for all $q$.

(e) Assume that $M$ is finitely generated. Then $N^{*}=N^{* *}$ for all $N \subseteq M$.

(f) $\left(N \cap N^{\prime}\right)^{*} \subseteq N^{*} \cap N^{\prime *}$.

(g) $\left(N+N^{\prime}\right)^{*}=\left(N^{*}+N^{\prime *}\right)^{*}$.

(h) $(I N)^{*}=\left(I^{*} N^{*}\right)^{*}$.

(i) If $J$ is the nilradical of $R, N^{*}$ contains $J M$ for every $N \subseteq M$.

(j) If $J$ is the nilradical of $R$, for any $N \subseteq M$, if $N^{\prime}$ denotes the image $(N+J M) / J M$ of $N$ in $M / J M$, then $N^{*}$ is the inverse image in $M$ of $\mathrm{Cl}\left(N^{\prime}, M / J M\right)$, where the last tight closure may be computed over either $R$ or $R_{\text {red }}$.

(k) If $N$ is tightly closed in $M$, then $N:{ }_{M} I$ (respectively, $N:{ }_{R} N^{\prime}$ ) is tightly closed in $M$ (respectively, $R$ ) for every ideal $I$ of $R$ (respectively, submodule $N^{\prime}$ of $M$ ).

(l) If $x \in \mathrm{Cl}(N, M)$, then $x^{q} \in \mathrm{Cl}\left(N^{[q]}, F^{e}(M)\right)$ for all $q=p^{e}$.

(m) If $N_{1}, \ldots, N_{h}$ are submodules of $M_{1}, \ldots, M_{h}$, respectively, $M=\bigoplus M_{i}$ and $N=\bigoplus N_{i} \subseteq M$, then $\mathrm{Cl}(N, M)=\bigoplus \mathrm{Cl}\left(N_{i}, M_{i}\right)$. In particular, $\mathrm{Cl}(0, M)$ $=\oplus \mathrm{Cl}\left(0, M_{i}\right)$.

Proof. (a) and (b) are straightforward. To prove (c), we first prove the final statement. The "if" part is clear. Suppose that $x \in N^{*}$, i.e., for some $c \in R^{\circ}$, $c x^{q} \in N^{[q]}$ for all $q \geq q^{\prime}$. We know there exists $c^{\prime} \in R^{\circ}$ such that $c^{\prime} x \in N$. Then $c^{\prime q^{\prime}} c x^{q} \in N^{[q]}$ for all $q$. The case where Ann $M / N$ has positive height is now obvious. Suppose, finally, that $R$ is reduced. If we localize at the multiplicative system $R^{\circ}, R$ becomes a finite product of fields. After localizing, we have that $x_{1}^{q} \in N_{1}^{[q]}$ for large $q$, where the subscript indicates images after localization. Over a finite product of fields, the Frobenius endomorphism is faithfully flat, and so we can conclude that $x_{1} \in N_{1}$, i.e., that we can choose $c^{\prime} \in R^{\circ}$ with $c^{\prime} x \in N$. (c) is therefore proved.

The proofs of $(\mathrm{d}),(\mathrm{e}),(\mathrm{f}),(\mathrm{g})$, and $(\mathrm{h})$ are now essentially the same as their counterparts in Proposition (4.1). We can deduce (i) from (h) with $I=(0)$. $N^{*} \supseteq 0^{*}=((0) M)^{*} \supseteq(0)^{*} M \supseteq J M$.

To prove $(\mathrm{j})$, first choose a fixed integer $q^{\prime \prime}$ such that $J^{\left[q^{\prime \prime}\right]}=0$. Note that this implies $(J M)^{\left[q^{\prime \prime}\right]}=0$ (which may be used to give a different proof of (i)). Note that when $M, N$ are modules over $R_{\text {red }}$, then $N_{M}^{[q]}$ is ambiguous. It may be computed over $R$ or over $R_{\text {red }}$. We shall write $N_{\text {red }}^{[q]}$ for the latter. We first observe that when $N \subseteq M$ are modules over $R_{\text {red }}, N^{*}$ is the same whether calculated over $R_{\text {red }}$ or over $R$. The point is that $F^{e}(M)$ calculated over $R$ becomes $F^{e}(M)$ over $R_{\text {red }}$ upon tensoring with $R_{\text {red }}$, and so $N^{[q]}$ maps onto $N_{\text {red }}^{[q]}$. The kernel of the map $N^{[q]} \rightarrow N_{\text {red }}^{[q]}$ is $J F^{e}(M) \cap N^{[q]}$. It is then clear that if $c x^{q} \in N^{[q]}$ (over $R$ ) for all $q \geq q^{\prime}$, then $c^{\prime} x^{q} \in N_{\text {red }}^{[q]}$ over $R_{\text {red }}$ for all $q \geq q^{\prime}$, where $c^{\prime}$ is the image of $c$ in $R_{\text {red }}$. Conversely, if $c^{\prime} x^{q} \in N_{\text {red }}^{[q]}$ for all $q \geq q^{\prime}$ (calculated over $R_{\text {red }}$ ), then $c x^{q} \in N^{[q]}+J F^{e}(M)$ for all $q \geq q^{\prime}$. 
But then $c^{q^{\prime \prime}} x^{q q^{\prime \prime}} \in N^{\left[q q^{\prime \prime}\right]}$. The rest of the argument uses the same trick and is omitted.

We next establish (k). We need only consider the case where $I=R x$ (respectively, $N^{\prime}=R x$ ), since an intersection of tightly closed submodules is tightly closed. Suppose that $u$ is in the tight closure of the colon. Then for some $c \in R^{\circ}$ we have that $c u^{q}$ is in $(N: R x)^{[q]}$, which is the $R$-span of elements $w^{q}$ with $w$ in $N: R x$. It follows that $c u^{q} x^{q}$ (or $c x^{q} u^{q}$ ) is in $N^{[q]}$ for all $q \gg 0$, and so $u x$ (or $x u$ ) is in $N^{*}=N$, i.e., $u \in N: R x$, as required.

To prove (1), note that if $c x^{q} \in N^{[q]}$ for all $q \geq q^{\prime}$, then $c\left(x^{q}\right)^{q^{\prime \prime}}=c x^{q q^{\prime \prime}} \in$ $N^{\left[q q^{\prime \prime}\right]}=\left(N^{[q]}\right)^{\left[q^{\prime \prime}\right]}$ for all $q^{\prime \prime} \geq q^{\prime}$.

We leave (m) as an exercise, except to note that in proving $\bigoplus \mathrm{Cl}\left(N_{i}, M_{i}\right) \subseteq$ $\mathrm{Cl}(N, M)$, one multiplies the individually chosen $c_{i}$ together.

Henceforth, in this section, we keep to our usual convention that modules are finitely generated unless otherwise specified.

All of the results proved for ideals and tight closure earlier have counterparts for modules, including the results on iterated operations. We shall not pursue this here, however. Instead, we focus on applications of tight closure for submodules peculiar to the case of modules, particularly results on vanishing of maps of homology (see $\S 9$ ). We must first develop several additional basic facts and explain how the theory of test elements developed in $\S 6$ extends to handle the module case.

(8.6) Discussion. Recall that a local ring $(R, m)$ is approximately Gorenstein if there is a sequence of irreducible $m$-primary ideals $Q_{t}$ cofinal with the powers of $m$. It is shown in [Ho5] that if $\operatorname{dim} R>0$, then $R$ is approximately Gorenstein iff its depth is positive and there does not exist a prime $Q$ of $\widehat{R}$ such that $\operatorname{dim} \widehat{R} / Q=1$ and $\widehat{R} / Q \oplus \widehat{R} / Q$ can be embedded in $\widehat{R}$. In particular, any local ring of depth 2 is approximately Gorenstein, and so every normal local ring is approximately Gorenstein. Moreover, every excellent reduced local ring is approximately Gorenstein, as is every excellent local ring that has no embedded prime of dimension one. All in all, the condition that a ring be approximately Gorenstein is quite weak.

(8.7) Proposition. Let $R$ be a ring of characteristic $p$ such that every ideal is tightly closed, i.e., $R$ is weakly F-regular. Then every submodule $N$ of a finitely generated module $M$ is tightly closed.

Proof. Let $x \in M$ be an element of $M$ not in $N$. We must show that $x$ is not in $N^{*}$. We may replace $N$ by a submodule of $M$ maximal with respect to not containing $x$, and we may replace $M, N$, and $x$ by $M / N, 0$, and $x+N$. From the fact that $x$ is in every nonzero submodule of $M$, it readily follows that $x$ is killed by a maximal ideal $m$ of $R$ and so $M$ is an essential extension of $K=R / m$, which implies that $M$ is killed by a power of $m$. Since $R$ is normal, $R_{m}$ is approximately Gorenstein, and there exists an irreducible $m$-primary ideal $Q \subseteq$ Ann $M$. The Artin ring $R / Q$ is self-injective, and $M$ is an essential extension of $K$ as an $(R / Q)$-module. It follows that $M$ can be 
embedded in $R / Q$. It will then suffice to show that 0 is tightly closed in $R / Q$, i.e., that $Q$ is tightly closed in $R$.

The proof of this proposition shows the following.

(8.8) Proposition. Let $Q_{t}$ be a sequence of irreducible m-primary ideals in the local ring $(R, m)$ of characteristic $p$ cofinal with the powers of $m$. In order that $R$ be weakly F-regular, it is necessary and sufficient that each of the ideals $Q_{t}$ be tightly closed.

In a different direction, we note the following.

(8.9) Proposition. Let $R$ be a ring of characteristic $p$, and let $N \subseteq M$ be finitely generated modules such that $M / N$ is killed by a power of a maximal ideal $m$ of $R$. Then $\left(N_{m}\right)^{*}=\left(N^{*}\right)_{m}$.

Proof. As in the proof of Proposition (4.14), it suffices to show $\subseteq$. Let $x$ be an element of $N$ such that $x / 1 \in\left(N_{m}\right)^{*}$. Exactly as in the proof of Proposition (4.14) we can choose $c \in R^{\circ}$ such that $(c / 1)(x / 1)^{q} \in N_{m}^{[q]} \cong N_{m}^{[q]}$. Since $\left(c x^{q}\right) / 1 \in N_{m}^{[q]}$ for all $q \gg 0, c x^{q} \in N^{[q]}$, since $F^{e}(M) / N^{[q]}$ is killed by a power of $m$.

Remark. Instead of assuming that $M / N$ is killed by a power of $m$, suppose that $x$ is an element of $M$ whose image in $M / N$ is killed by a power of $m$. Suppose also that $x$ is in the tight closure of $N_{m}$ in $M_{m}$. Exactly the same argument shows that $x$ is in the tight closure of $N$ in $M$. The only change needed is to replace $F^{e}(M) / N^{[q]}$ by $(N+R x)^{[q]} / N^{[q]}$ in the last line of the proof.

(8.10) Discussion. In $\S 6$, we introduced the notion of a $q^{\prime}$-weak test element. From this point on, we shall refer to these as " $q$ '-weak test elements for ideals," for we are about to introduce a new version of the notion for the module case. So long as the ring is mildly well behaved (e.g., if its local rings are approximately Gorenstein) and $q^{\prime}=1$, we can prove that the two notions coincide. In general, a $q^{\prime}$-weak test element for modules also is a $q^{\prime}$-weak test element for ideals, but we have been unable either to prove or disprove the converse. The results in the two theories are entirely parallel. In every instance where we can construct (weak) test elements for ideals, we can also construct them for modules.

(8.11) Definition. Let $R$ be Noetherian ring of characteristic $p$. We say that $c \in R^{\circ}$ is a $q^{\prime}$-weak test element if for every finitely generated module $M$ and submodule $N, x \in M$ is in $N^{*}$ iff $c x^{q} \in N_{M}^{[q]}$ for all $q \geq q^{\prime}$. We say that $c$ is a locally (respectively, completely) stable $q^{\prime}$-weak test element if its image in (respectively, in the completion of) every local ring of $R$ is a $q^{\prime}$-weak test element.

(8.12) Remarks. In the definition, it suffices to consider the case where $N=0$; it also suffices to consider instead the case where $M$ is free. Moreover, if $c x^{q} \in N^{[q]}$ for all $q \geq q^{\prime}$, then it is automatic that $x \in N^{*}$. It is the "only if" part that is significant, for if $c x^{q} \notin N^{[q]}$ for even one value of $q \geq q^{\prime}$, we can 
conclude that $x \notin N^{*}$. Note that the condition $c x^{q} \in N^{[q]}$ for all $q \geq q^{\prime}$ can be rephrased as $c x^{q q^{\prime}} \in N^{\left[q q^{\prime}\right]}$ for all $q$.

As indicated earlier, the theory of test elements for ideals generalizes without essential change to the module case. We sketch the main points rapidly. We first note the following analogue of Proposition (6.1).

(8.13) Proposition. Let $R$ be Noetherian of characteristic $p$ and $c \in R$.

(a) $c$ is a $q^{\prime}$-weak test element for $R$ iff $c / 1$ is a $q^{\prime}$-weak test element for $R_{m}$ for every maximal ideal $m$ of $R$.

(b) If $R$ has a $q^{\prime}$-weak test element, then the tight closure of $N \subseteq M$ is the intersection of the tightly closed submodules $N^{\prime} \supseteq N$ such that $M / N^{\prime}$ is killed by a power of a maximal ideal.

(c) If $c \in R$ is a $q^{\prime}$-weak test element for the completion $\widehat{R}$ of the local ring $R$, then it is also a $q^{\prime}$-weak test element for $R$. In this case, for all $N \subseteq M$ and $x \in M, x \in N^{*}$ if and only if $x \in(\widehat{N})^{*}$, i.e., $N^{*}=(\widehat{N})^{*} \cap M$.

(d) Let $J$ be the ideal of nilpotents in $R$ and suppose that $J^{\left[q^{\prime \prime}\right]}=0$. Let $c^{\prime}$ be the image of $c \in R$ in $R_{\mathrm{red}}$. If $c$ is a $q^{\prime}$-weak test element for $R$, then $c^{\prime}$ is a $q^{\prime}$-weak test element in $R_{\mathrm{red}}$. If $c^{\prime}$ is a $q^{\prime}$-weak test element in $R_{\mathrm{red}}$, then $c^{q^{\prime \prime}}$ is a $q^{\prime} q^{\prime \prime}$-weak test element in $R$.

Proof. (a) Suppose that $c$ is a $q^{\prime}$-weak test element for $R$. Suppose $N \subseteq M$, $x / 1 \in\left(N_{m}\right)^{*}$, and $q \geq q^{\prime}$. We must show that $c x^{q} / 1 \in N_{m}^{[q]}$. If not, we can choose a positive integer $t$ such that $c x^{q} / 1 \notin m^{t} M_{m}+N_{m}^{[q]}$ and then $c x^{q} / 1 \notin\left(m^{t} M+N\right)^{[q]} R_{m}$, which implies that $c x^{q} \notin\left(m^{t} M+N\right)^{[q]}$ and hence that $x \notin\left(m^{t} M+N\right)^{*}$, since $c$ is a $q^{\prime}$-weak test element in $R$. But then Proposition (8.9) implies that $x / 1 \notin\left(m^{t} M_{m}+N_{m}\right)^{*}$ and so $x / 1 \notin N_{m}{ }^{*}$, a contradiction.

Conversely, suppose that $c / 1$ is a $q^{\prime}$-weak test element for $R_{m}$ for every ideal $m$ of $R$. This implies $c \in R^{\circ}$. Suppose $N \subseteq M, x \in N^{*}$, and $q \geq q^{\prime}$ but $c x^{q} \notin N^{[q]}$. Then we can localize at a maximal ideal $m$ of $R$ so as to preserve the last condition, and we still have $x / 1 \in N_{m}{ }^{*}$, which contradicts the condition that $c / 1$ be a $q^{\prime}$-weak test element for $R_{m}$.

(b) One inclusion is obvious. To prove the other, suppose that $x \in M-$ $N^{*}$. We must construct $N^{\prime} \supseteq N$ such that $M / N^{\prime}$ is killed by a power of a maximal ideal and $x \notin N^{\prime *}$. Since $x \notin N^{*}$, we can choose $q \geq q^{\prime}$ such that $c x^{q} \notin N^{[q]}$. We can choose $m$ such that $c x^{q} / 1 \notin N_{m}^{[q]}$ and then $t$ such that $c x^{q} / 1 \notin m^{t} M_{m}+N_{m}^{[q]}$, and we then conclude exactly as in the proof of part (a) that $x \notin\left(m^{t} M+N\right)^{*}$.

(c) Since $\widehat{R}$ is $R$-flat, $R^{\circ} \subseteq \widehat{R}^{\circ}$, and so $N^{*} \subseteq(\widehat{N})^{*}$ for $N \subseteq M$. Let $c \in R$ be a $q^{\prime}$-weak test element for $\widehat{R}$. The results claimed all follow if we can prove that for all $N \subseteq M$ and $x \in M$, if $x \in(\widehat{N})^{*}$, then $c x^{q} \in N^{[q]}$ for all $q \geq q^{\prime}$. But since $c$ is a $q^{\prime}$-weak test element for $\widehat{R}$, we have $c x^{q} \in \widehat{N}^{[q]}=\left(N^{[q]}\right)^{-}$ and since $\widehat{R}$ is faithfully flat over $R$, this implies that $c x^{q} \in N^{[q]}$, as required. 
(d) The essential trick in the argument has already been given in the discussion of part (j) of Proposition (8.5).

We can now observe that the statement and proof of Corollary (6.2) apply verbatim to the case of modules. Theorem (6.9) likewise generalizes. Since some changes in statements are needed, we give an explicit version.

(8.14) Theorem. Let $R$ be torsion-free, module-finite, and generically smooth over a regular domain $A \subseteq R$. Let $c \in A^{\circ}$ be such that $c R^{\infty} \subseteq A^{\infty}$. Then the following conditions on finitely generated $R$-modules $N \subseteq M$ and an element $x \in M$ are equivalent.

(a) $x \in N^{*}$.

(b) For every maximal ideal $m$ of $A, x \in N_{m}{ }^{*}$.

(c) For every maximal ideal $m$ of $A, x \in \widehat{N}^{m *}$ in $\widehat{M}^{m}$, where $\widehat{M}^{m}$ denotes the $m$-adic completion of $M$ and is isomorphic to $\hat{A}_{m} \otimes_{A} M$, where $\hat{A}_{m}$ denotes the completion of the local ring $A_{m}$ (which is isomorphic with $\hat{A}^{m}$ ).

$\left(c^{\circ}\right)$ For every maximal ideal $m$ of $R, x \in \widehat{N}_{m}{ }^{*}$ in $\widehat{M}_{m}{ }^{*}$, where $\widehat{M}_{m}$ denotes the completion of $M_{m}$.

(d) Let $G$ be a finitely generated free module mapping onto $M$, let $H$ be the inverse image of $N$, and let $y$ be an element of $G$ that maps to $x$. Then for every maximal ideal $m$ of $A$ there exists a sequence of elements $\left\{\varepsilon_{n}\right\}_{n}$ in $\left(\left(\widehat{R}^{m}\right)^{\infty}\right)^{\circ}$ such that $\operatorname{ord}\left(\mathbf{N}\left(\varepsilon_{n}\right)\right) \rightarrow 0$ as $n \rightarrow \infty$ and $\varepsilon_{n} y \in H\left(\hat{R}^{m}\right)^{\infty}$, the $\left(\widehat{R}^{m}\right)^{\infty}$-submodule of $G \otimes_{R}\left(\widehat{R}^{m}\right)^{\infty}$ spanned by $H$. ( $\mathbf{N}$ is the norm from $\left(\widehat{R}^{m}\right)^{\infty}$ to $\hat{A}_{m}$ described in (6.6); ord is the function described in (6.8).)

(e) $c x^{q} \in N^{[q]}$ for all $q$ of the form $p^{e}$.

Proof. There is no loss of generality in assuming that $M$ is free (we can replace $N, M$ by $H, G$ as in the statement of part (d)). As in the proof of Theorem (6.9), we show (a) $\Rightarrow(\mathrm{b}) \Rightarrow(\mathrm{c}) \Rightarrow$ (d) $\Rightarrow$ (e) $\Rightarrow$ (a) and (c) $\Leftrightarrow\left(\mathrm{c}^{\circ}\right)$. The argument is nearly the same. We discuss only the most interesting implication, (d) $\Rightarrow$ (e), and that only minimally.

Assume (d) but that there exists $e$ such that $c x^{q} \notin N^{[q]}$, where $q=p^{e}$. Exactly as in the proof of Theorem (6.9), we can reduce to the case where $A$ is complete local. The rest of the argument is the same except $N$ replaces $I$ throughout.

Next, we remark that Theorem (6.13), Lemma (6.16), Theorem (6.17), and Corollaries (6.18) and (6.19) extend to the case of modules with no changes whatsoever in wording and no significant changes in their proofs. Likewise, Proposition (6.20) is valid for modules. The proof is the same for $N \subseteq R^{t}$ as for $I \subseteq R$, and one then obtains Proposition (6.23) and Corollary (6.24) for the module case as well.

The next result establishes our earlier assertion that test elements for ideals are automatically test elements for modules in the approximately Gorenstein case.

(8.15) Proposition. Let $R$ be a Noetherian ring of characteristic $p$ whose local rings are approximately Gorenstein. Let $c \in R^{\circ}$ be a test element for ideals in 
$R$. Then $c$ is a test element (for modules). Moreover, if $c$ is (completely) stable as a test element for ideals, it is also (completely) stable as a test element (for modules).

Proof. First note that the final statement is immediate from the earlier one, since the hypothesis is retained in passing to the (completed) localization.

Rephrased slightly, the problem is to show that if $x \in N^{*}$, then for all $q=p^{e}, c x^{q} \in N^{[q]}$. Since $x \in N^{*}{ }_{M}, x^{q} \in N_{F^{e}(M)}^{[q] *}$. We replace $N$, $M, x$ by $N^{[q]}, F^{e}(M), x^{q}$. Thus, it suffices to show that if $x \in N_{M}^{*}$, then $c x \in N$. If this fails, we can replace $N$ by a submodule of $M$ containing $N$ and maximal with respect to the property that $c x \notin N$. We can now replace $M, N$ by $M / N$ and 0 , and we are then in the situation where $N=0$ and $M$ is an essential extension of $R / m$ for a suitable maximal ideal $m$ of $R$. Since $R_{m}$ is approximately Gorenstein, we can choose an $m$-primary irreducible ideal $I$ such that $M$ embeds into $R / I$. It will still be true that $x$ is in the tight closure of 0 in $R / I$. Let $y \in R$ lift $x$. But then $y \in I^{*}$ in $R$, and so $c y \in I$ in $R$ and $c x$. is 0 in $R / I$ and hence in $M$.

Our next main result, Theorem (8.17), relates when an element is in the tight closure of a submodule to the growth of certain lengths as iterations of the Frobenius endomorphism are applied. We use $l$ to denote length.

We need the following lemma first.

(8.16) Lemma. Let $N \subseteq M$ be finitely generated modules over a Noetherian ring $R$ of characteristic $p$ that has a $q^{\prime}$-weak test element $c \in R^{\circ}$. Let $x \in M$, and suppose there exists $d \in R^{\circ}$ such that $d x^{q} \in N^{[q] *}$ in $F^{e}(M) \quad\left(=M^{[q]}\right)$ for infinitely many values of $q$. Then $x \in N_{M}^{*}$. Hence, if $x \notin N_{M}^{*}$, then $d x^{q} \notin N^{[q] *}$ (in $\left.F^{e}(M)\right)$ for all $q \gg 0$.

If $R$ is reduced, $M$ is free, and $N^{[q]} R^{\infty}$ denotes the submodule of $F^{e}(M) \otimes_{R} R^{\infty}$ spanned by the image of $N^{[q]}$, then $x \notin N_{M}^{*} \Rightarrow d x^{q} \notin N^{[q]} R^{\infty}$ for all $q \gg 0$.

Proof. To establish the first part, we shall show that $c^{q^{\prime}+1} d^{q^{\prime}} x^{q} \in N^{[q]}$ for all $q \gg 0$. First note that whenever $d x^{q} \in N^{[q] *}$, we have $c d^{q^{\prime}} x^{q q^{\prime}} \in N^{\left[q q^{\prime}\right]}$, since $c$ is a $q^{\prime}$-weak test element. Now, given any $q$, we can choose a larger one, say $q Q q^{\prime}$, such that $c d^{q^{\prime}} x^{q Q q^{\prime}} \in N^{\left[q Q q^{\prime}\right]}$, and so $\left(c d x^{q}\right)^{Q q^{\prime}} \in\left(N^{[q]}\right)^{\left[Q q^{\prime}\right]}$. Hence, for all $Q^{\prime}, 1\left(c d x^{q}\right)^{Q q^{\prime} Q^{\prime}} \in\left(N^{[q]}\right)^{\left[Q q^{\prime} Q^{\prime}\right]}$, which shows that $c d x^{q} \in N^{[q] *}$ for all $q$. But then, since $c$ is a $q^{\prime}$-weak test element, $c\left(c d x^{q}\right)^{q^{\prime}} \in N^{[q]\left[q^{\prime}\right]}$ for all $q$, i.e., $c^{q^{\prime}+1} d^{q^{\prime}} x^{q^{\prime} q} \in N^{\left[q^{\prime} q\right]}$ for all $q$, which shows that $x \in N^{*}$.

The last statement now follows from the observation that if $V$ is free and $W \subseteq V$, then $W R^{\infty} \cap V \subseteq W_{V}^{*}$ applied with $W=N^{[q]}$ and $V=F^{e}(M)$, for if $v \in W R^{1 / q^{\prime}} \cap V$, then $v^{q^{\prime}} \in W^{\left[q^{\prime}\right]}$ and, as above, $1 v^{q^{\prime} q^{\prime \prime}} \in W^{\left[q^{\prime} q^{\prime \prime}\right]}$ for all $q^{\prime \prime}$.

(8.17) Theorem (length criterion for tight closure). Let $R$ be a Noetherian ring of characteristic $p$, and let $N \subseteq W \subseteq M$ be finitely generated modules 
such that $W / N$ is killed by a power of a maximal ideal $m$ of $R$. Let $d$ be the height of $m$.

(a) If $W \subseteq N_{M}^{*}$, then for all $q, l\left(W_{M}^{[q]} / N_{M}^{[q]}\right) \leq C q^{d-1}$ for some constant $C$ independent of $q$.

(b) Conversely, if $R_{m}$ is analytically unramified (i.e., its completion is reduced) and formally equidimensional (i.e., its completion is equidimensional) and has a completely stable $q_{1}$-weak test element $c$ (or a $q_{1}$-weak test element $c$ that is also one for $\left.\left(R_{m}\right)^{\sim}\right)$ and if $\liminf _{q \rightarrow \infty} l\left(W_{M}^{[q]} / N_{M}^{[q]}\right) / q^{d}=0$, then $W \subseteq N_{M}^{*}$. Hence, in this case,

$$
\text { (i) } \begin{aligned}
W \subseteq N_{M}^{*} & \Leftrightarrow \text { (ii) } l\left(W_{M}^{[q]} / N_{M}^{[q]}\right) \leq C q^{d-1} \text { for some } C \text { and all } q \\
& \Leftrightarrow \text { (iii) } \lim _{q \rightarrow \infty} l\left(W_{M}^{[q]} / N_{M}^{[q]}\right) / q^{d}=0 \\
& \Leftrightarrow \text { (iv) } \liminf _{q \rightarrow \infty} l\left(W_{M}^{[q]} / N_{M}^{[q]}\right) / q^{d}=0 .
\end{aligned}
$$

Proof. (a) Choose $c \in R^{\circ}$ such that $c W^{[q]} \subseteq N^{[q]}$ for all $q \gg 0$. Let $J$ be an $m$-primary ideal such that $J W \subseteq N$. Let $\bar{b}$ bound the number of generators of $W$. Then $W^{[q]} / N^{[q]}$ has at most $b$ generators and is killed by $J^{[q]}+c R$, so that

$$
\begin{aligned}
l\left(W^{[q]} / N^{[q]}\right) & \leq b l\left(R /\left(J^{[q]}+c R\right)\right) \\
& =b l\left(R_{m} /\left(c R+J^{[q]}\right)_{m}\right) .
\end{aligned}
$$

Let $S=R_{m} / c R_{m}$. Since $c \in R^{\circ}, \operatorname{dim} S \leq d-1$. Now $l\left(W^{[q]} / N^{[q]}\right) \leq$ $b l\left(S / J^{[q]} S\right)$. If $I=\left(y_{1}, \ldots, y_{d-1}\right) S$, where $y_{1}, \ldots, y_{d-1}$ is a s.o.p. for $S$ in $J S$, the latter number is bounded by $b l\left(S / I^{[q]}\right) \leq b l(S / I) q^{d-1}$, since $S / I^{[q]}$ has a filtration with $q^{d-1}$ factors, each of which is a homomorphic image of $S / I$.

(b) (i) $\Rightarrow$ (ii) follows from (a) and (ii) $\Rightarrow$ (iii) $\Rightarrow$ (iv) is obvious. Hence, it will suffice to show that (iv) $\Rightarrow$ (i). Assume (iv) and suppose that there is an element $x \in W$ such that $x \notin N^{*}$. It will suffice to show that there is a constant $\gamma>0$ such that $l\left(W_{M}^{q a]} / N_{M}^{[q]}\right) \geq \gamma q^{d}$ for all $q \gg 0$. By the remark following Proposition (8.9), we may replace $R, N, W, M$ by their localizations at $m$, and $x$ by its image in $W_{m}$. The remark guarantees that we still have $x \notin N_{M}^{*}$. Note that Frobenius commutes with localization and that the lengths we are discussing will not change. Hence, we may assume that $(R, m)$ is local. Choose $q \geq q_{1}$ such that $c x^{[q]} \notin N^{[q]}$. This condition will be preserved if we complete $R$, and so the condition $x \notin N^{*}$ is preserved if we complete. The length condition is unaffected. Thus, we henceforth assume that $(R, m)$ is complete local, reduced, and equidimensional. It is then modulefinite and torsion-free over a complete regular local domain $A$. Moreover, we can choose $q^{\prime \prime}$ such that $S=R\left[A^{1 / q^{\prime \prime}}\right] \subseteq R^{\infty}$ is separable over $A^{1 / q^{\prime \prime}}$. By the results of $\S 6$, we can choose $d \in A^{\circ}$ such that $d S^{1 / q} \subseteq S\left[A^{1 / q q^{\prime \prime}}\right]$ for all $q$. Moreover, $R^{\infty}=S^{\infty}$ is flat over $S\left[A^{1 / q q^{\prime \prime}}\right]$. 
Without loss of generality, we can replace $M$ by a finitely generated free module mapping onto it and $N, W$ by their inverse images in the free module. Thus, we may assume that $M$ is free. Since $x \in W$ is not in the tight closure of $N$, by Lemma (8.16) we can choose $q^{\prime}$ such that $d x^{q} \notin N^{[q]} R^{\infty}$ for all

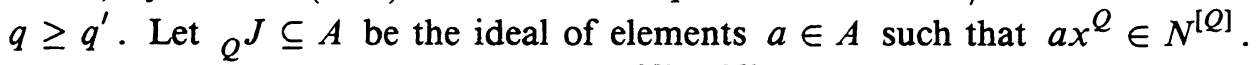
We first note that $A / Q^{J}$ injects into $W^{[Q]} / N^{[Q]}$ (the map is $A$-linear). Simply send the class of $a$ to the class of $a x^{Q}$. Since $A$ and $R$ have the same residue class field, it will suffice to show that there is a constant $\gamma>0$ such that $l\left(A /_{Q} J\right) \geq \gamma Q^{d}$ for all $Q \gg 0$.

Assume that $Q \geq q^{\prime} q^{\prime \prime}$, and write $Q=q q^{\prime} q^{\prime \prime}$. Then $a \in \in_{Q} J \Rightarrow a x^{Q} \in$ $N^{[Q]} \Rightarrow a^{1 / q q^{\prime \prime}} x^{q^{\prime}} \in N^{\left[q^{\prime}\right]} R^{1 / q q^{\prime \prime}}$ (i.e., the submodule spanned by $N^{\left[q^{\prime}\right]}$ inside the free module $\left.M^{\left[q^{\prime}\right]} \otimes_{R} R^{1 / q q^{\prime \prime}}\right) \Rightarrow d a^{1 / q q^{\prime \prime}} x^{q^{\prime}} \in N^{\left[q^{\prime}\right]}\left(d S^{1 / q}\right) \subseteq N^{\left[q^{\prime}\right]} S\left[A^{1 / q q^{\prime \prime}}\right] \cong$ $N^{\left[q^{\prime}\right]} S \otimes_{S} S\left[A^{1 / q q^{\prime \prime}}\right]$ (since $S\left[A^{1 / q q^{\prime \prime}}\right]$ is $S$-flat). We thus conclude that $a^{1 / q q^{\prime \prime}} \in$ $\left(N^{\left[q^{\prime}\right]} S \otimes_{S} S\left[A^{1 / q q^{\prime \prime}}\right]\right): d x^{q^{\prime}} \cong\left(N^{\left[q^{\prime}\right]} S:{ }_{S} d x^{q^{\prime}}\right) S\left[A^{1 / q q^{\prime \prime}}\right]$, again using the flatness of $S\left[A^{1 / q q^{\prime \prime}}\right]$ over $S$. Now $N^{\left[q^{\prime}\right]} S:{ }_{S} d x^{q^{\prime}} \subseteq N^{\left[q^{\prime}\right]} R^{1 / q^{\prime \prime}}: d x^{q^{\prime}}$ since $S \subseteq$ $R^{1 / q^{\prime \prime}}$. By the choice of $q^{\prime}, d x^{q^{\prime}} \notin N^{\left[q^{\prime}\right]} R^{1 / q q^{\prime \prime}}$, and so $N^{\left[q^{\prime}\right]} S:{ }_{S} d x^{q^{\prime}}$ is contained in the maximal ideal $m^{1 / q^{\prime \prime}}$ of $R^{1 / q^{\prime \prime}}$. This shows that $a^{1 / q q^{\prime \prime}} \in$ $m^{1 / q^{\prime \prime}} R^{1 / q q^{\prime \prime}} \Rightarrow a \in m^{[q]}$. Thus, if $Q \geq q q^{\prime} q^{\prime \prime}$, then $Q^{J \subseteq m^{\left[Q / q^{\prime} q^{\prime \prime}\right]}} R \cap A$. We can choose an integer $D$, still a power of $p$, such that $m^{D} \subseteq m_{A} R$, where $m_{A}$ is the maximal ideal of $A$, and then

$$
m^{\left[Q / q^{\prime} q^{\prime \prime}\right]} R \cap A \subseteq m^{Q / q^{\prime} q^{\prime \prime}} R \cap A \subseteq m_{A}^{Q / q^{\prime} q^{\prime \prime} D} R \cap A \subseteq m_{A}^{\left(Q / q^{\prime} q^{\prime \prime} D\right)-t}
$$

for a certain constant $t$, by the Artin-Rees lemma, and for large $Q$ this is $\subseteq m_{A}^{Q / B}$, where $B=q^{\prime} q^{\prime \prime} D p$. But then $l\left(A / Q^{J}\right) \geq l\left(A / m_{A}^{Q / B}\right)$, and the latter is polynomial in $Q / B$ of degree $d$ with leading coefficient $1 / d !$. We may then choose $\gamma$ to be any positive real number less that $1 /\left(d ! B^{d}\right)$.

We shall see that this length characterization of tight closure combined with the phantom acyclicity criterion of the next section yields a powerful tool. We pursue this further in [HH4], where we give a new proof of the improved new intersection theorem (in fact, of a much stronger result) using these ideas.

The next proposition is aimed at showing that the tight closure of a submodule of a projective module over a normal ring is independent of how it is embedded in a projective module.

(8.18) Proposition. Let $R$ be a reduced Noetherian ring of characteristic $p$, and $M, N, F, G$ be finitely generated modules.

(a) If $M / N$ is torsion-free, then $N$ is tightly closed in $M$. More generally, $N_{M}^{*}$ may be identified with a submodule of $N^{\prime}=\operatorname{Ker}\left(M \rightarrow\left(R^{\circ}\right)^{-1}(M / N)\right)$. If $N$ is torsion-free, $N^{\prime} \subseteq\left(R^{\circ}\right)^{-1} N$.

(b) If $N \subseteq G \subseteq F$, where $G$ is projective and $F$ is any module, then $N_{F}^{*} \cap G=$ $N_{G}^{*}$. Hence, if $G$ is tightiy closed in $F$, then $N_{F}^{*}=N_{G}^{*}$. 
(c) If $R$ is normal, and $G \subseteq F$ with $G$ projective and $F$ torsion-free, then $G$ is tightly closed. If an arbitrary module $N$ has embeddings in two possibly distinct finitely generated projective modules $F$ and $G$, then $N_{F}^{*} \cong N_{G}^{*}$ canonically.

Proof. (a) For the first statement, we may assume that $N=0$, and that $M$ is torsion-free, and so embeddable in a free module. But 0 is tightly closed in $R$, and so in any free module, since $R$ is reduced. The second statement is immediate from the first, and the third statement from the second.

(b) Suppose, to the contrary, that $x$ is an element of $G$ in $N_{F}^{*}$ but not in $N_{G}^{*}$. By localizing at a suitable maximal ideal (one containing $N_{G}^{*}: x$ ) we may assume that $(R, m)$ is local and that $G$ is free. By killing a maximal submodule of $F$ disjoint from $G$, we may assume that $G \subseteq F$ is essential. This implies that $F$ is torsion-free of the same rank as $G$, and so $F$ can be embedded in a free module so that the extension is essential. Thus, there is no loss of generality in assuming that $F$ is free of the same rank as $G$. Identify $F$ and $G$ with $R^{h}$, and let the embedding $G \subseteq F$ be given by a size $h$ matrix $A=\left[a_{i j}\right]$ with $\operatorname{det}(A) \in R^{\circ}$. We can think of the Frobenius endomorphism as acting coordinatewise in $F$ and $G$ and identify $G^{[q]} \subseteq F^{[q]}=F^{e}(F)$ with the map $R^{h} \subseteq R^{h}$ given by $A^{[q]}$. The hypothesis that $x \in R^{h}=G$ is in $N_{F}^{*}$ translates to the statement that for some $c \in R^{\circ}, c(A x)^{q} \in(A N)_{F}^{[q]}$ for all $q \gg 0$ or $A^{[q]}\left(c x^{q}\right) \in A^{[q]}\left(N_{G}^{[q]}\right)$. Since $\operatorname{det}\left(A^{[q]}\right)=\operatorname{det}(A)^{q}, A^{[q]}$ is one-to-one, and so $c x^{q} \in N_{G}^{[q]}$ for all $q \gg 0$, as required.

The second statement in (b) is then immediate.

(c) Suppose that $x \in F$ is in the tight closure of $G$ but not in $G$. This is preserved when we localize at a minimal prime of $G: x$. Hence, we may assume that $(R, m)$ is local normal and that the image of $x$ in $F / G$ is killed by a power of $m$. Since $F$ is torsion-free, $0 \rightarrow G \rightarrow G+R x \rightarrow R /(G: x) \rightarrow 0$ cannot split if $\operatorname{dim} R>0$ and so represents an element of $\operatorname{Ext}_{R}^{1}(R /(G: x), G)$ that is nonzero. But the Ext vanishes if $\operatorname{dim} R \geq 2$, since $G$ is free and $R$ is normal. It follows that $\operatorname{dim} R \leq 1$. But then, since $R$ is normal, $R$ is regular, and $G$ is tightly closed, a contradiction.

The last part of (c) is then obtained as follows. Given embeddings of $N$ into $F, G$, we can form a pushout $F \oplus_{N} G$, that contains both $F$ and $G$. The torsion in this module meets neither $F$ nor $G$. Embed the quotient in a free module $D$. It then suffices to compare $N_{F}^{*}$ (resp. $N_{G}^{*}$ ) with $N_{D}^{*}$. Thus, there is no loss of generality in assuming that $G \subseteq F$. Since $G$ is tightly closed in $F$ by the first part of (c), the second part of (b) shows that $N_{G}^{*}=N_{F}^{*}$ when $G \subseteq F$. The uniqueness of the isomorphism follows from the last statement in (a).

We next want to define the absolute tight closure of a module $N$ in a module $M$. In this definition, we allow modules that are not finitely generated. We first introduce a variant of the notion of tight closure that has advantages in considering non-Noetherian modules. 
(8.19) Definition. If $R$ is a Noetherian ring of characteristic $p$ and $N \subseteq M$, we define $N_{M}^{* f g}$, the finitistic tight closure of $N$ in $M$, as the union, taken over the finitely generated submodules of $M^{\prime}$ of $M$, of the modules $\left(M^{\prime} \cap N\right)_{M^{\prime}}^{*}$.

(8.20) Definition. If $R$ is a Noetherian ring of characteristic $p$ and $N \subseteq M$, we define $N_{M}^{*}$ abs , the absolute tight closure of $N$ in $M$, to be the set of all elements $x \in M$ such that for some module $Q \supseteq M, x \in N_{Q}^{* f g}$.

(8.21) Discussion. First note that $N^{* \text { abs }}$ is a submodule. This follows from the fact that any two extensions $Q, Q^{\prime}$ of $M$ are contained in a common extension $Q \oplus_{M} Q^{\prime}$. In fact, if $E$ is any injective module containing $M$, e.g., an injective hull of $M$, then $N_{M}^{* \text { abs }}=N_{E}^{* f g} \cap M$. For suppose $x \in M$ is in $N_{Q}^{* f g}$ for $Q \supseteq M$. Then this is also true with $Q$ replaced by $Q \oplus_{M} E$. Thus, we might as well assume that $Q \supseteq E$. But then $Q=E \oplus W$, and any finitely generated submodule of $Q$ is contained in the sum of a finitely generated submodule of $E$ and a finitely generated submodule of $W$. The rest of the argument is straightforward. We now connect these ideas with the theory of test elements and the ideal they generate.

(8.22) Definition. Let $R$ be a Noetherian ring of characteristic $p$. We define the test ideal $\tau(R)$ of $R$, as $\bigcap_{M} \mathrm{Ann}_{R} 0_{M}^{*}$, where $M$ runs through all finitely generated $R$-modules. For each prime ideal $P$, we define $\tau(R ; P)$ as $\bigcap_{M} \operatorname{Ann}_{R} 0_{M}^{*}$, where $M$ runs through all finitely generated $R$-modules whose only associated prime is $P$.

(8.23) Proposition. (a) $c \in \tau(R)$ if and only if whenever $N \subseteq M$ and $x \in N_{M}^{*}$, then $c x^{q} \in N_{M}^{[q]}$ for all $q$.

(b) $R$ has a test element iff $\tau(R)$ is not contained in any minimal prime of $R$. In this case, $\tau(R)$ is the ideal generated by the test elements, and $\tau(R) \cap R^{\circ}$ is the set of test elements.

(c) Let $E_{R}(R / P)=E(R / P)$ denote an injective hull over $R$ of the prime cyclic module $R / P$. Then $\tau(R ; P)=$ Ann $0_{E(R / P)}^{* f g}$.

(d) $\tau(R)=\bigcap_{m} \tau(R ; m)=\operatorname{Ann}_{R} 0_{E}^{* f g}$, where $E=\bigoplus_{m} E(R / m)$, and $m$ runs through all maximal ideals of $R$. In particular, if $R$ is local, then $\tau(R)=$ $\tau(R ; m)$.

(e) If $(R, m)$ is local and $R$ has a $q^{\prime}$-weak test element that is also a $q^{\prime}$-weak test element for $\widehat{R}$, then $\tau(R)=\tau(\widehat{R}) \cap R$.

(f) If $(R, m)$ is local and $\left\{I_{t}\right\}$ is a sequence of m-primary irreducible ideals cofinal with the powers of $m$, then $\tau(R)=\bigcap_{t} \operatorname{Ann}_{R}\left(I_{t}^{*} / I_{t}\right)=\bigcap_{t} I_{t}: I_{t}^{*}$ (where $I_{t}^{*}$ is the tight closure of $I_{t}$ in $R$ ).

(Such a sequence exists iff $R$ is approximately Gorenstein.)

Proof. (a) Suppose $c x^{q} \in N_{M}^{[q]}$ for all $q$ whenever $x \in N_{M}^{*}$ for a finitely generated $M$. Applying this when $N=0$ and $q=1$, we see at once that $c \in \tau(R)$. For the converse, suppose that $c \in \tau(R)$ and $x \in N_{M}^{*}$. We must show that $c x^{q} \in N_{M}^{[q]}$ for all $q$. Since $x \in N_{M}^{*}, x^{q} \in N_{M^{[q]}}^{[q] *}$. But then, applying 
the definition of $\tau(R)$ and noting that $x^{q}+N^{[q]}$ is in the tight closure of 0 in $M^{[q]} / N^{[q]}$, we see that $c x^{q} \in N^{[q]}$.

(b) is then immediate from (a) and the definition of test element.

(c) The key point is that any finitely generated module whose only associated prime is $P$ can be embedded in a finite direct sum of such modules each of which is an essential extension of $R / P$, and these can be taken to be the same. It follows that $\tau(R ; P)$ is the intersection of $\operatorname{Ann}\left(0_{M}^{*}\right)$ taken over all finitely generated modules $M$ such that $M$ is an essential extension of $R / P$. Since $E(R / P)$ contains every other essential extension, this is the same as Ann $0_{E(R / P)}^{* f g}$.

(d) It is clear that $\tau(R) \subseteq \tau(R ; P)$ for every prime $P$. Now suppose that $c \in \tau(R ; m)$ for every maximal ideal $m$ but that $c \notin \tau(R)$. Then there exist a finitely generated module $M$ and an element $x \in 0_{M}^{*}$ such that $c x \neq 0$. Choose $N \subseteq M$ maximal with respect to not containing $c x$, and replace $M$ by $M / N$ and $x$ by $x+N$. The new $M$ is killed by a power of a maximal ideal $m$, and we obtain $c \notin \tau(R ; m)$, a contradiction.

(e) The modules of finite length over $R$ are identical with those over $\widehat{R}$. When $M$ has finite length, $F^{e}(M)$ is independent of whether it is calculated over $R$ or $\widehat{R}$. The existence of the $q^{\prime}$-weak test element that can be used for tests both over $R$ and over $\widehat{R}$ implies that the notion of tight closure for submodules of modules of finite length over $R$ is the same as over $\widehat{R}$. The result now follows because $\tau(R)=\tau(R ; m)$ is the intersection of a certain family of $m$-primary ideals in $R$ (the ideals $\operatorname{Ann}_{R} 0_{M}^{*}$ for finite length $M$ ), $\tau(\widehat{R})$ is the intersection of the extensions to $\widehat{R}$ (or completions) of these ideals, and every ideal of $R$ is contracted from $\widehat{R}$.

(f) If $M$ is an essential extension of $R / m$ of finite length with annihilator $J \supseteq I_{t}$, then $M$ can be embedded in $R / I_{t}$, and $\operatorname{Ann}_{R}\left(0_{M}^{*}\right) \supseteq \operatorname{Ann}_{R}\left(0_{R / I_{t}}^{*}\right)$. But the tight closure of 0 in $R / I_{t}$ is $I_{t}^{*} / I_{t}$.

The notion of absolute tight closure enables us to define several left exact functors on $R$-modules. The derived functors appear to be quite interesting. We first need the following observation.

(8.24) Proposition. Let $R$ be a Noetherian ring of characteristic $p$, and let $h: M \rightarrow N$ be a homomorphism of arbitrary (not necessarily finitely generated) $R$-modules. Let $W$ be any submodule of $M$.

(a) $h\left(W_{M}^{* \text { abs }}\right) \subseteq h(W)_{N}^{* \text { abs }}$.

(b) If $h: M \subseteq N$ is an inclusion, $W_{M}^{* \text { abs }}=W_{N}^{* \text { abs }} \cap M$.

Proof. The map $h$ extends to a map $h^{\prime}$ of injective hulls $E(M) \rightarrow E(N)$ by virtue of the injectivity of $E(N)$, which is an injection in case (b) (because $M \rightarrow E(M)$ is essential). Since $W_{M}^{* \text { abs }}$ is $W_{E(M)}^{* f g} \cap M$, and $h^{\prime}$ maps $W_{E(M)}^{* f g}$ into $h(W)_{E(N)}^{* f g}$ and $M$ into $N$, it follows that $h\left(W_{M}^{* \text { abs }}\right) \subseteq h(W)_{N}^{* \text { abs }}$. The second part follows from the observation that $E(M)$ is a direct summand of $E(N)$. 
(8.25) Discussion and definitions. It follows that if $L$ is a left exact functor that assigns to each $R$-module a submodule of itself and acts on maps by restriction (e.g., the zero functor, the functor that, for some fixed ideal $I$, sends $M$ to $\mathrm{Ann}_{M} I\left(\cong \operatorname{Hom}_{R}(R / I, M)\right)$, or the local cohomology functor $H_{I}^{0}$ (whose value on $M$ is $\left.\bigcup_{t} \operatorname{Ann}_{M} I^{t}\right)$ ), we can define a new functor, $L^{\text {abs }}$, whose value on $M$ is the absolute tight closure of $L(M)$ in $M$. It is immediate from Proposition (8.24) that $L^{\text {abs }}$ is a left exact functor, and so has right derived functors $R^{i} L^{\text {abs }}$. These are of interest even if $L$ is the zero functor! For in that case $L^{\text {abs }}(M)$ is $0_{M}^{* \text { abs }}$. When $L$ is a zero functor, we shall write $\mathrm{ABS}^{i}$ for $R^{i} L^{\text {abs }}$. Note that if $R$ is weakly F-regular, then $\operatorname{ABS}^{i}(M)=0$ for $i \geq 1$.

(8.26) Definition. We say that a local ring $R$ of characteristic $p$ is $\tau$-complete if $R$ has a test element and $\tau(R)^{-}=\tau(\widehat{R})$.

We conjecture, but cannot prove, that the local rings for which we can prove the existence of test elements are $\tau$-complete. Of course, any complete local ring is $\tau$-complete.

(8.27) Proposition. If $(R, m)$ is $\tau$-complete and $M$ has finite length, then $\operatorname{ABS}^{i}(M) \cong \operatorname{Ext}_{R}^{i}(R / \tau(R), M)$ as functors of $M$.

Proof. The key point is that $M$ has an injective resolution by injectives each of which is a direct sum of copies of $E(R / m)$. It therefore suffices to show that $0_{E(M)}^{*}$ is the same as $\operatorname{Ann}_{E(M)} \tau(M)$, and our hypotheses allow us to pass to the case where $R$ is complete. In this case, taking annihilators gives bijections, in both directions, between submodules of $E(R / m)$ and ideals of $R$. Since $\mathrm{Ann}_{R} 0_{E(R / m)}^{*}=\tau(R), \mathrm{Ann}_{E(R / m)} \tau(R)=0_{E(R / m)}^{*}$, as required.

(8.28) Remark. If we do not know that $R$ is $\tau$-complete but we do know, at least, that $R$ has a test element that is a test element in $\widehat{R}$, we still have $\operatorname{ABS}^{i}(M)=\operatorname{Ext}_{\widehat{R}}^{i}(\widehat{R} / \tau(\widehat{R}), M)$ for $M$ of finite length, by the same argument.

\section{Phantom homology AND THE PHANTOM ACYCLICITY CRITERION IN CHARACTERISTIC $p$}

In this section, we introduce the notion of phantom (co)homology for a complex of modules over a Noetherian ring of characteristic $p$. A complex has phantom homology at a given spot if the cycles are in the tight closure of the boundaries. See the detailed definitions below. We give criteria for phantom acyclicity for free complexes (this means that the positively indexed homology is phantom) by proving parallels of the Buchsbaum-Eisenbud criterion for acyclicity of free complexes [BE]. However, in order to do this, we must prove "acyclicity" lemmas with denominators. We do not assume that complexes are actually acyclic, but keep track of an element, or a power of it, that kills homology. In fact, not only an element acts, but a power of Frobenius as well. The role of depth is replaced by $(\phi, c)$-depth, where $\phi$ is an endomorphism of $R$ (the identity when $R$ does not have characteristic $p$, and the identity or a power of the Frobenius endomorphism if $R$ does have characteristic $p$ ). This means that the $c \phi$ kills a certain family of Koszul homology modules. When 
$\phi$ is the identity and $c=1$, this notion coincides with ordinary depth, and the theory developed here then specializes to the situation of [BE].

In $\S 9$, all of this worked out under the assumption that $R$ is a locally equidimensional quotient of a Cohen-Macaulay ring.

In $\S 10$, we introduce a family of alternative notions of tight closure that includes our original notion. One of these is used to give a new proof of a generalization of the syzygy theorem. In $\S 11$, we give improved versions of some of the results of this section under the stronger hypothesis that $R$ is a homomorphic image of a Gorenstein ring of finite Krull dimension. One of the payoffs is that one knows in this situation that if $R_{c}$ is Cohen-Macaulay, then $c$ has a power $c^{\prime}$ that kills the higher homology of any complex $G$. of free modules that satisfies the phantom acyclicity criterion Theorem (9.8), and all of the higher homology images $F^{e} G$. under iterations of Frobenius as well.

In [HH4], we study the notion of the minheight of a module on an ideal, and introduce the notion of a weakly Cohen-Macaulay ring or module. This enables us to prove analogues of the phantom acyclicity criterion for free complexes tensored with an arbitrary module, as well as to obtain results when the module or ring is mixed. The point is to work with minheight instead of height, and likewise, to work with $c$ such that $R_{c}$ is weakly Cohen-Macaulay. These results, as well as some very general acyclicity criteria with denominators, are developed in the early part of [HH4], where we then use our theory of phantom acyclicity to prove several extraordinarily powerful vanishing theorems.

(9.1) Definition. Let $G$. (respectively, $G^{*}$ ) be a nonnegative left (respectively, right) complex of finitely generated modules over a Noetherian ring $R$ of characteristic $p$. We say that the $i$ th homology (respectively, cohomology) module $H_{i}(G$.$) (respectively, H^{i}\left(G^{*}\right)$ ) is phantom if every element in the kernel $Z_{i}$ of $G_{i} \rightarrow G_{i-1}$ (respectively, $Z^{i}$ of $G^{i} \rightarrow G^{i+1}$ ) is in the tight closure, within $G_{i}$ (respectively, $G^{i}$ ), of the image $B_{i}$ of $G_{i+1} \rightarrow G_{i}$ (respectively, $B^{i}$ of $G_{i-1} \rightarrow G_{i}$ ). If this holds for all $i \geq 1$, we say that $G$. (respectively, $G^{*}$ ) has phantom homology (respectively, cohomology), or is phantom acyclic.

We shall say that an element of a homology module of a complex is phantom if it is represented by a cycle that is in the tight closure of boundaries in the appropriate module of the complex. In this case, every representative cycle is in the tight closure of the boundaries. Thus, a homology module is phantom iff every element is phantom.

(9.2) Remark. Note that $H_{i}(G$.) is phantom if and only if it is in the tight closure of 0 in $G_{i} / B_{i}$.

One of the key points about phantom homology is

(9.3) Theorem. Let $R, S$ be Noetherian rings of characteristic $p$. Let $G$. be a complex of finitely generated $R$-modules. Suppose that $R \rightarrow S$ maps $R^{\circ}$ to $S^{\circ}$. If $H_{i}\left(G_{.}\right)$is phantom and $S$ is weakly $F$-regular, then the induced map $H_{i}(G.) \rightarrow H_{i}\left(G . \otimes_{R} S\right)$ is zero. The same result holds for cohomology.

The result also holds for any complex $G^{\prime}$. of finitely generated $S$-modules to which G. maps. 
If $R$ has a (weak) test element, the condition that $R^{\circ}$ map to $S^{\circ}$ may be weakened to the condition that at least one (weak) test element in $R$ map to $S^{\circ}$.

This rather easy result will produce a multitude of powerful vanishing theorems when coupled with the fact that the Koszul homology of a system of parameters is always phantom under extremely mild conditions on the ring. In order to prove Theorem (9.3), we establish a more general result.

(9.4) Theorem. Let $R, S$ be Noetherian rings of characteristic $p$. Let $G$. be a complex of finitely generated $R$-modules, and let $G^{\prime}$. be such a complex of $S$-modules. Suppose that $R \rightarrow S$ maps $R^{\circ}$ to $S^{\circ}$, or else suppose that $R$ has a weak test element that maps into $S^{\circ}$. Suppose also that a map $\phi_{.}: G . \rightarrow G^{\prime}$. is given. If $x \in H_{i}\left(G_{.}\right)$is phantom, then its image in $H_{i}\left(G^{\prime}\right)$ is phantom. If every submodule of $G_{i}^{\prime}$ is tightly closed, then the image of $x$ is 0 . In particular, if $S$ is weakly F-regular, then the image of $x$ is 0 . The same result holds for cohomology.

Proof. Let $z \in Z_{i}$ represent $x$. We know that $c z^{q} \in B_{i}^{[q]}$ for all $q \gg 0$, with $c \in R^{\circ}$ or with $c$ the weak test element specified. The map $G . \rightarrow G^{\prime}$. induces a map $F^{e}(R) \otimes_{R} G_{i} \rightarrow F^{e}(S) \otimes_{S} G_{i}^{\prime}$, i.e., of $F^{e}\left(G_{i}\right)$ (over $\left.R\right) \rightarrow F^{e}\left(G_{i}^{\prime}\right)$ (over $S$ ). Since $\phi\left(B_{i}\right) \subseteq B_{i}^{\prime}$, it follows that $\phi(c) \phi(z)^{q} \in B_{i}^{\prime[q]}$ for all $q \gg 0$. This establishes the first statement. The remaining statements are clear. Over a weakly F-regular ring, every submodule of a finitely generated module is tightly closed.

(9.5) Discussion. Our next objective is to prove an analogue of the BuchsbaumEisenbud criterion for the acyclicity of a complex (which is closely related to the Peskine-Szpiro acyclicity lemma; see [BE] and [PS1]). The BuchsbaumEisenbud criterion guarantees the acyclicity of a free complex under certain conditions when specific ideals associated with it have sufficiently large depths. Our results assert that the complexes have phantom homology $H_{i}$ for positive $i$ when the same ideals have sufficiently large heights. This yields very strong vanishing theorems when the complex is mapped to a complex over an F-regular ring.

The criterion we give is stable under application of $F^{e}$ and so implies that not only the original complex, but also all of its images under tensoring with Frobenius, have phantom homology. (Complexes that have phantom homology can, in the general case, lose this property under tensoring with iterations of Frobenius. See Example (9.19).) We shall show that, modulo nilpotents, a criterion similar to that of [BE] but with height replacing depth is necessary and sufficient for a finite nonnegative free complex and all its images under tensoring with iterations of Frobenius to have phantom homology in positive degree. Some hypothesis on the ring is necessary. As indicated in more detail in the introduction to this section, various generalizations of this result are developed in $\S \S 10,11$, and the early sections of [HH4]. The most striking applications are given in [HH4].

(9.6) Notation. In this paragraph, we fix some notation to which we refer repeatedly below. Let $R$ be a Noetherian ring, not necessarily of characteristic 
$p$, and let $G$. be a complex of finitely generated free modules

$$
0 \rightarrow G_{n} \rightarrow G_{n-1} \rightarrow \cdots \rightarrow G_{i} \rightarrow \cdots \rightarrow G_{1} \rightarrow G_{0} \rightarrow 0 .
$$

Denote the map from $G_{i}$ to $G_{i-1}$ by $\alpha_{i}$. Let $b_{i}$ denote the rank of $G_{i}$, with the convention $b_{i}=0$ if $i>n$ or $i<0$, and let $r_{i}=\sum_{t=i}^{n}(-1)^{t-i} b_{t}$, $1 \leq i \leq n$, while $r_{n+1}=0$. The $r_{i}$ are the unique integers such that $r_{n+1}=0$ and $r_{i+1}+r_{i}=b_{i}, 1 \leq i \leq n$.

By the rank of a map of free modules $\alpha: G \rightarrow G^{\prime}$ we mean the largest integer $r$ such that the induced map $\Lambda^{r} \alpha: \Lambda^{r} G \rightarrow \Lambda^{r} G^{\prime}$ is not zero. $I_{t}(\alpha)$ denotes the ideal generated by the size $t$ minors of a matrix for $\alpha$. It is independent of the choice of bases for $G$ and $G^{\prime}$. By convention, $I_{0}(\alpha)=R$, even when $\alpha$ is the zero map between modules, one or both of which are zero, while $I_{t}(\alpha)=(0)$ if $t$ exceeds the rank of either the domain or the target of the map $\alpha$. Thus, rank $\alpha$ is the same as the largest integer $r$ such that $I_{r}(\alpha) \neq 0$.

Many times throughout this paper we refer to "a complex as in (9.6)." This automatically introduces all the notation, conventions, and conditions of the above paragraphs. This will be convenient because both the criterion of [BE] for the acyclicity of a free complex and our various phantom acyclicity criteria are applied primarily to complexes of the type described.

We comment on two differences from [BE]. One is that we allow maps to be zero, which corresponds to allowing the numbers $\operatorname{rank} \alpha_{i}$ to be zero. Our convention about $I_{0}(\alpha)$ yields correct results in this case. Second, for simplicity, we have tended to restrict our treatment to free complexes. Since the results are local, one gets immediate extensions to the case of projective modules which, for the most part, we have not bothered to state. However, in [HH4], we go to an opposite extreme, and consider complexes in substantially greater generality than when the modules are projective. Instead, we place a condition on the "codimension" (in an appropriate sense, which may be that of depth, c-depth, height, or minheight) of the non-locally-free locus). We have purposely confined this level of technicality to one section in [HH4]. The reader can bypass most of that material if he is interested only in the case of free complexes over locally equidimensional rings.

(9.7) Discussion and definitions. We say that a complex as in (9.6) satisfies the standard condition on rank, if, for $1 \leq i \leq n$, $\operatorname{rank} \alpha_{i}=r_{i}$ (equivalently, $\left.b_{i}=\operatorname{rank} \alpha_{i+1}+\operatorname{rank} \alpha_{i}, 1 \leq i \leq n\right)$. We say that a complex as in (9.6) satisfies the standard condition for depth (respectively, height) if the depth (respectively, the height) of the ideal $I_{i}=I_{r_{i}}\left(\alpha_{i}\right)$ is at least $i, 1 \leq i \leq n$. Note that the depth (and height) of the ideal $I$ is $+\infty$ if $I=R$ while if $I$ is proper its depth is the length of any maximal $R$-sequence contained in $I$. Thus, the depth or height condition is satisfied whenever $I_{i}$ is the unit ideal. Recall that if $r=0$, we make the convention that $I_{r}(\alpha)$ is the unit ideal.

(Later we shall consider functions on ideals taking values in $\mathbf{N} \cup\{+\infty\}$ other than depth and height. E.g., we can consider the depth, height, or minheight (this is defined in [HH4]) of an ideal on a fixed module. We can then say that $G$. satisfies the standard condition for, say, depth on $N$, meaning that the depth of $I_{i}$ on $N$ is $\geq i, 1 \leq i \leq n$.) 
With this terminology, the acyclicity criterion of $[\mathrm{BE}]$ asserts that a complex as in (9.6) is acyclic iff it satisfies the standard conditions for rank and depth. (There is also a version for the result of tensoring $G$. with a module. We return to this point in [HH4].) The results we obtain on acyclicity with denominators will recover this result.

We can now state one of the results we are aiming for, although we shall not be able to give the proof until several preliminary results have been established.

(9.8) Theorem (phantom acyclicity criterion). Let $R$ be a Noetherian ring of characteristic $p$. Suppose that $R$ is a homomorphic image of a Cohen-Macaulay ring and is locally equidimensional. Let $G$. be a free complex over $R$ with notation as in (9.6). Let $\alpha_{i}^{\text {red }}$ be the result of tensoring with $R_{\text {red }}$. Suppose that $\operatorname{rank} \alpha_{i}^{\mathrm{red}}=r_{i}, 1 \leq i \leq n$ (equivalently, $b_{i}=\operatorname{rank} \alpha_{i+1}^{\mathrm{red}}+\operatorname{rank} \alpha_{i}^{\mathrm{red}}, 1 \leq i \leq n$ ), and suppose that the height of the ideal $I_{i}=I_{r_{i}}\left(\alpha_{i}\right)$ is at least $i, 1 \leq i \leq n$. Then $H_{i}\left(F^{e} G\right.$.) is phantom for all $e \in \mathbf{N}$ and all $i \geq 1$.

Conversely, let $R$ be an arbitrary Noetherian ring of characteristic $p>0$, and let $G$. be a free complex over $R$ with notation as in (9.6). If $H_{i}\left(F^{e} G\right.$.) is phantom for all $e \in \mathbf{N}$ and all $i \geq 1$, then $\operatorname{rank} \alpha_{i}^{\mathrm{red}}=r_{i}, 1 \leq i \leq n$, and the height of $I_{i}=I_{r_{i}}\left(\alpha_{i}\right)$ is at least $i, 1 \leq i \leq n$.

(9.9) Discussion and definitions. The development of the tools needed to prove this result and the proof itself will occupy most of the rest of this section.

Before proceeding further, we introduce the following terminology. Let $R$ be any Noetherian ring, not necessarily of characteristic $p$. Let $G$. be a free complex as in (9.6). We shall say that $G$. satisfies the phantom acyclicity criterion if $G_{.}^{\text {red }}$ satisfies the standard rank condition, and $G$. (or, equivalently, $G^{\text {red }}$ ) satisfies the standard height condition.

Notice that if $G$. satisfies the phantom acyclicity criterion, it continues to do so after tensoring with any ring $S$ such that ht $J S \geq$ ht $J$ for all ideals $J$ of $R$. This condition is automatic if $S$ is an integral extension of $R$ (it need not be an extension provided that the elements that map to 0 are nilpotent) or if $S$ is flat over $R$, e.g., a localization. If $S=R$ and the map is a power of the Frobenius endomorphism, the question of whether the phantom acyclicity criterion holds is unaffected. Thus, if we show that satisfying the criterion is sufficient for phantom acyclicity for $G_{\text {. }}$, it is clear that it will be sufficient for phantom acyclicity for $F^{e} G$. for all $e \in \mathbf{N}$. It is then clear that in order to have the converse hold, we must impose the condition that $F^{e} G$. have phantom homology for $i \geq 1$ for all $e \geq 0$. It is easy to give examples where $G$. is phantom, but this is not preserved when we apply Frobenius. See Example (9.19) below.

With this new terminology, we can restate Theorem (9.8) as follows.

Theorem $(9.8)^{\circ}$. Let $G$. be a free complex as in (9.6), and suppose $R$ is Noetherian of characteristic $p$. If $H_{i}\left(F^{e} G\right.$.) is phantom for all $i \geq 1$ and for all $e \geq 0$, then $G$. satisfies the phantom acyclicity criterion. If $R$ is locally equidimensional and a homomorphic image of a C-M ring, then $H_{i}\left(F^{e} G\right.$.) is phantom for all $i \geq 1$ and all $e$ if and only if $G$. satisfies the phantom acyclicity criterion. 
One of the tools we shall use in the proof is a technical generalization of the sufficiency of the Buchsbaum-Eisenbud criterion that is a refinement of the following observation. Suppose that the hypothesis of the Buchsbaum-Eisenbud criterion is satisfied after localizing at $c$. Then there is a power of $c$ that kills the homology of $G_{\text {. }}$. Note that this statement is a trivial consequence of the Buchsbaum-Eisenbud criterion. What we want to do is set up the hypothesis and conclusion in such a way that the power of $c$ in the conclusion is bounded in a manner independent of the maps in the complex, so that, in characteristic $p$, the same power can be used even after applying the Frobenius endomorphism.

We can handle this by assuming that $c$ kills the higher Koszul homology of sequences of elements of appropriate lengths in the ideals $I_{i}$ arising in the Buchsbaum-Eisenbud criterion, and also the homology of the sequences formed by taking their powers. Unfortunately, this result is not strong enough to achieve the theorem we want. For technical reasons, we need to consider a situation in which $c$ kills the image of the Koszul homology of the type described above after tensoring with a certain fixed power of the Frobenius endomorphism. The point is that we can construct elements $c$ with this weaker property for a large class of rings. For this reason, we need a sort of Buchsbaum-Eisenbud criterion with "denominators" (the denominator in question is $c$ ) in which a certain fixed endomorphism of the ring is also allowed to play a role. In the applications in this paper, the endomorphism in question will turn out to be either the identity or else some fixed positive power of the Frobenius endomorphism.

In $\S 11$, by limiting attention to images of finite-dimensional Gorenstein rings, we are able to show that it is not necessary to use the fixed power of Frobenius referred to above. One can kill off homology as necessary simply by multiplying by a suitable element. However, we need the more general set-up to handle homomorphic images of C-M rings. In this section, we consider only complexes of free modules, and we assume that the ring is locally equidimensional. In [HH4], we discuss how the theory can be extended to more general settings.

(9.10) Discussion and notation. In order to make a precise statement, we need to be careful about the sense in which one achieves the required depths upon localizing at $c$. Let $R$ be an arbitrary Noetherian ring, not necessarily of characteristic $p$. Let $\phi$ be a fixed endomorphism of $R$. Let $\Phi^{n}(R)$ denote $R$ viewed as an $(R, R)$-bimodule where the left action is given by the identity map and the right action by $\phi^{n}$. Given a left $R$-module $M$, we write $\Phi^{n}(M)$ for the left $R$-module $\Phi^{n}(R) \otimes_{R} M$. Note that $\Phi^{n}(R) \cong R$ and $\Phi^{n}\left(\right.$ Coker $\left.\left[a_{i j}\right]\right) \cong$ Coker $\left[\phi^{n}\left(a_{i j}\right)\right]$. When $\phi=F, \Phi^{n}$ is precisely the Peskine-Szpiro functor $F^{n}$. If $\mathbf{x}=x_{1}, \ldots, x_{i}$ is a sequence of elements of $R$, let $\mathbf{x}^{t}=x_{1}^{t}, \ldots, x_{i}^{t}$, and let $H_{j}\left(\mathbf{x}^{t} ; M\right)$ denote the $j$ th Koszul homology module of the $R$-module $M$ with respect to $\mathbf{x}^{t}$. Note that $H_{j}\left(\mathbf{x}^{t} ; R\right)$ maps into $H_{j}\left(\phi^{n}(\mathbf{x})^{t} ; \Phi^{n}(R)\right)$ (the map is not $R$-linear but is $\phi^{n}$-linear). If $d \in R$ is such that $d$ kills the image of this map, we shall say that " $d \Phi^{n}$ kills $H_{j}\left(\mathbf{x}^{t} ; R\right)$." More generally, for any complex G., $H_{j}\left(G\right.$.) maps $\left(\phi^{n}\right.$-linearly) to $H_{j}\left(\Phi^{n}\left(G_{.}\right)\right)$, and if $d$ kills the image, we shall say that " $d \Phi^{n}$ kills $H_{j}(G$.$) ."$ 
(9.11) Definition. Let $\phi$ be an endomorphism of a Noetherian ring $R$ that is either the identity map or else a power of the Frobenius endomorphism. We shall say that an ideal $I \subseteq R$ has $(\phi, c)$-depth $\geq n$ on an $R$-module $M$ if for every integer $i$ with $1 \leq i \leq n$, there are elements $x_{1}, \ldots, x_{i} \in I$ such that for every integer $j \geq 1$ and for every integer $t \geq 1, c \Phi$ kills $H_{j}\left(x_{1}^{t}, \ldots, x_{i}^{t} ; M\right)$. If $\phi$ is the identity, we may omit it from the notation and use the term $c$-depth. If $M=R$, we refer to the " $(\phi, c)$-depth of $I$ " instead.

(9.12) Remarks. If $\phi$ is the identity and $c=1$, this condition is equivalent to the condition depth $M \geq n$. In general, when $\phi$ is the identity, it implies that depth $I_{c} M_{c} \geq n$. However, it is a substantially stronger condition, since it bounds $a$ priori (with bound equal to one) the exponent on $c$ needed to obtain a power of $c$ that kills all the Koszul homology. It is not clear, in general, that one can use a power that is independent of $t$.

If $c=1$ and $\phi$ is a power of Frobenius, it is not so clear what the condition means. For example, the ideal $I$ will have $\left(F^{e}, 1\right)$-depth $\geq n$ for large $e$ provided either that the depth of $I$ on $R$ or the depth of $I R_{\text {red }}$ on $R_{\text {red }}$ is at least $n$.

We next need to introduce some functions. If $n$ is a nonnegative integer, we shall define $\square n$ recursively by the rules $\square 0=1$ and $\square(n+1)=\square n+$ $\sum_{t=0}^{n} \square t+n+2$. Thus, $\square 1=1+1+2=4, \square 2=4+(4+1)+3=12$, $\square 3=12+(12+4+1)+4=33$, etc. (It is routine to show that $\square n=f_{2 n+2}-1$, where $f_{n}$ denotes the $n$th Fibonacci number. $f_{0}=f_{1}=1$ and $f_{n+1}=f_{n}+f_{n-1}$, $n \geq 1$. We shall make no use of this fact.)

If $q$ is a positive integer and $n \in \mathbf{N}$, we shall write $q\langle n\rangle$ for $\sum_{t=0}^{n-1} q^{t}$. Thus, $q\langle 0\rangle=0,1\langle n\rangle=n$, and, of course, $q\langle n\rangle=\left(q^{n}-1\right) /(q-1)$ if $q>1$.

If $\phi$ is either the identity homomorphism on $R$ or else the $e$ th iteration of the Frobenius endomorphism, and we let $q^{\prime}=1$ in the former case and $q^{\prime}=p^{e}$ in the latter case, then for any element $c \in R$ we have, by a trivial induction on $N$, that $(c \Phi)^{N}=c^{q^{\prime}\langle N\rangle} \Phi^{N}$ as maps from a complex $G$. of $R$-modules to the complex $\Phi^{N} G_{\text {. }}$, and hence as maps of homology $H_{i}\left(G_{.}\right) \rightarrow H_{i}\left(\Phi^{N} G_{.}\right)$. Note that in the composition map $(c \Phi)^{N}$ the $j$ th copy of $c \Phi$ counting from the right should be interpreted as a map from $\Phi^{j-1} G$. to $\Phi^{j} G_{0}, 1 \leq j \leq N$. Thus, $(c \Phi)^{N}$ kills $H_{i}\left(G_{\text {. }}\right)$ if and only if $c^{q^{\prime}\langle N\rangle} \Phi^{N}$ kills $H_{i}\left(G_{\text {. }}\right)$, i.e., $c^{q^{\prime}\langle N\rangle}$ kills the image of $H_{i}\left(G_{\text {. }}\right)$ in $H_{i}\left(\Phi^{N}\left(G_{.}\right)\right)$.

We can now state one of our main technical lemmas. It can be viewed as a generalization of the sufficiency of the Buchsbaum-Eisenbud criterion for acyclicity. The original situation for the Buchsbaum-Eisenbud criterion is the case $c=1$, with $\phi$ the identity on $R$. The proof contains, as a special case, a new proof of the sufficiency of the Buchsbaum-Eisenbud criterion. In a certain sense, the argument is more "constructive" than previous ones.

(9.13) Theorem (free acyclicity criterion with denominators). Let $R$ be an arbitrary Noetherian ring and $\phi$ a fixed endomorphism that is either the identity or else a power of the Frobenius endomorphism. Let $\phi(c)=c^{q^{\prime}}$. $q^{\prime}$ may be 1 
(and must be 1 in characteristic 0). Let $G_{.}, b_{i}, r_{i}$, and $\alpha_{i}$ be as in (9.6). Suppose for $1 \leq i \leq n$ that $\operatorname{rank} \alpha_{i}=r_{i}$, and let $I_{i}=I_{r_{i}}\left(\alpha_{i}\right)$. Finally, suppose that the $(\phi, c)$-depth of $I_{i}$ is $\geq i$ for $1 \leq i \leq n$. Then $(c \Phi)^{\square t}=c^{q^{\prime}\langle\square t\rangle} \Phi^{\square t}$ kills $H_{n-t}(G),. 0 \leq t \leq n-1$.

The crucial point here is that the exponents $q^{\prime}\langle\square t\rangle$ and $\square t$ depend only on $q^{\prime}$ and $t$. In particular, if we apply an iteration of the Frobenius endomorphism, we can use the same exponents to kill homology no matter how high a power of Frobenius we apply.

Before giving the proof of this rather technical result, we want to show how it implies the sufficiency of the phantom acyclicity criterion, Theorem (9.8). We first need

(9.14) Theorem. (a) If $R$ has characteristic $p$, is a homomorphic image of a Cohen-Macaulay ring, and is locally equidimensional, there is an element $c \in R^{\circ}$ and a fixed integer $e^{\prime}$ such that for every ideal $I$ of $R$ the $\left(F^{e^{\prime}}, c\right)$-depth of $I$ is at least the height of $I$.

(b) If $R$ is an arbitrary Noetherian ring (i.e., not necessarily of characteristic $p), R$ is reduced, $R=S / Q$, where $S$ is Cohen-Macaulay, and $S_{q}$ is regular for each minimal prime $q$ of $Q$, and $R$ is locally equidimensional, then there is an element $c \in R^{\circ}$ such that for every ideal $I$ of $R$ the $\left(i d_{R}, c\right)$-depth of $I$ is at least the height of $I$.

Proof. We first give the proof of (a), and then describe the changes that need to be made to prove (b). If $R=R_{1} \times R_{2}$ and we have such a $c_{i}$ for each component $R_{i}$, then it is easy to see that the element $\left(c_{1}, c_{2}\right)$ solves the problem for $R$. It follows that there is no loss of generality in assuming that $\operatorname{Spec} R$ is connected. In this case, when we write $R=S / Q$ with $S$ Cohen-Macaulay, we know, as in the proof of Theorem (7.9), that $Q$ is equidimensional and hence that if $W$ is the complement of the set of minimal primes of $Q$, we can choose $z_{1}, \ldots, z_{d} \in$ $Q$ whose images in the semilocal ring $W^{-1} S$ are a system of parameters and such that $z_{1}, \ldots, z_{d}$ is an $S$-sequence. It follows that there is an element $c_{1} \in W$ and an integer $q^{\prime}=p^{e^{\prime}}$ such that $c_{1} Q^{\left[q^{\prime}\right]} \subseteq\left(z_{1}, \ldots, z_{d}\right) S \subseteq Q$. Let $c$ be the image of $c_{1}$ in $R$. Then $c$ has the required property. To see this, let $I$ be an ideal of height $n$ in $R$. It is easy to see that the inverse image of $I$ in $S$ has height $n+d$. It follows that $z_{1}, \ldots, z_{d}$ can be extended to a regular sequence $z_{1}, \ldots, z_{d}, x_{1}^{\prime}, \ldots, x_{n}^{\prime}$ in $I$. Let $x_{j}$ be the image of $x_{j}^{\prime}$ in $R$. Given a Koszul cycle $z$ in $K_{j}\left(\mathbf{x}^{t} ; R\right)$, we can lift it to an element $z^{\prime}$ of $K_{j}\left(\mathbf{x}^{t} ; R^{\prime}\right)$, where $R^{\prime}=S /\left(z_{1}, \ldots, z_{d}\right)$. It is now only a cycle modulo $Q K_{j}\left(\mathbf{x}^{t} ; R^{\prime}\right)$. However, $c_{1} z^{\prime\left[q^{\prime}\right]}$ is a cycle, and, hence, a boundary, since $\mathbf{x}$ and, hence, $\mathbf{x}^{t}$, is a regular sequence in $R^{\prime}$, and it follows that $c z^{\left[q^{\prime}\right]}$ is a boundary if $j \geq 1$. This is equivalent to the assertion that $c F^{e^{\prime}}$ annihilates $H_{j}\left(\mathbf{x}^{t} ; R\right)$ if $j \geq 1$.

(b) Let the minimal primes of $Q$ be $\left\{q_{i}\right\}$ and, as before, let $W=S-\bigcup_{i} q_{i}$. Then $W^{-1} S$ is regular, and $Q W^{-1} S$, which is an intersection of maximal 
ideals of the same height, say, $d$, is an ideal-theoretic complete intersection (this is clear after completion with respect to the Jacobson radical, which does not affect the issue). Moreover, by prime avoidance, it is possible to pick generators for $Q W^{-1} S$ in $Q$ that generate an ideal of height $d$ in $S$. (Suppose we choose $z_{1}, \ldots, z_{d} \in Q$ that generate $Q S_{q}$ for each $q=q_{i}$. Altering $z_{t}$ by an element in $Q^{2}$ does not affect this property, since $Q^{2} S_{q}=\left(q S_{q}\right)^{2}$ when $q=q_{i}$. It suffices then to observe that if $z_{1}, \ldots, z_{t}$ have been modified by elements in $Q^{2}$ so that they generate an ideal of height $t, t<d$, then $z_{t+1}$ can also be so modified. Otherwise, $z_{t+1}+Q^{2}$ would be contained in the union of the minimal primes of $\left(z_{1}, \ldots, z_{t}\right) S$, and then $Q^{2}$ would be contained in one of them (see Theorem 124 on p. 90 of [Kap]), contradicting the fact that height $Q^{2}=d$.)

With $z_{1}, \ldots, z_{d}$ constructed as above, since

$$
Q W^{-1} S=\left(z_{1}, \ldots, z_{d}\right) W^{-1} S,
$$

there is an element $c_{1} \in W$ with $c_{1} Q \subseteq\left(z_{1}, \ldots, z_{d}\right) S$. The rest of the argument is now precisely the same as in part (a), except that we can use $Q$ where we used $Q^{\left[q^{\prime}\right]}$, and we make no use of the Frobenius endomorphism.

Next we observe

(9.15) Lemma. Let $R$ be a Noetherian ring of characteristic $p$, and let $G$. be a free complex with notation as in (9.6).

(a) If $H_{i}\left(F^{e} G\right.$.) is phantom for all $i \geq 1$ and all $e \geq e^{\prime}$, then it is phantom for all $i \geq 1$ and all $e$. More precisely, if a cycle $z$ in $G_{i}$ has an image $z^{q^{\prime}}$ that is phantom in $H_{i}\left(F^{e^{\prime}} G\right.$.) for some $e^{\prime}$, then $z$ itself is phantom.

(b) Let $G^{\mathrm{red}}=G_{.} \otimes_{R} R_{\mathrm{red}}$. If $H_{i}\left(F^{e} G_{\text {. }}\right)$ is phantom for all $i \geq 1$ and all $e \in \mathbf{N}$, then $G^{\text {red }}$, thought of as a complex over $R_{\text {red }}$ has the same property.

(c) If $G$. satisfies the phantom acyclicity criterion, then for all sufficiently large $e, \operatorname{rank} F^{e}\left(\alpha_{i}\right)=r_{i}$.

(d) If $c \in R^{\circ}$ kills $H_{i}\left(F^{e} G\right.$.) for all $e$ (or for all sufficiently large $e$ ), then $H_{i}\left(F^{e} G\right.$.) is phantom for all $e$. If $R$ has a test element $c \in R^{\circ}$, then $H_{i}\left(F^{e} G\right.$.) is phantom for all $e$ iff $H_{i}\left(F^{e} G\right.$. $)$ is killed by $c$ for all $e$.

Proof. (a) It suffices to show that $H_{i}\left(G\right.$.) is phantom, $i \geq 1$, since every $F^{e} G$.

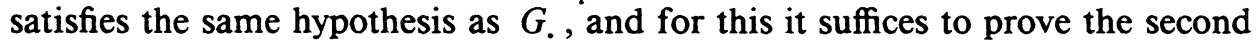
statement. Since $z^{q^{\prime}}$ in $F^{e^{\prime}} G$. is a cycle in the tight closure of the boundaries, there is a $c \in R^{\circ}$ such that $c\left(z^{q^{\prime}}\right)^{q}$ is a boundary for all sufficiently large $q$. Note that, by the right exactness of tensor, the module of boundaries in $F^{e^{\prime}+e} G$. may be identified with $B^{\left[q^{\prime}\right][q]}$, where $B$ is the module of boundaries in $Z_{i}$. It follows that $z$ is in the tight closure of $B$.

(b) If suffices to show that $H_{i}\left(G^{\text {red }}\right)$ is phantom over $R_{\text {red }}$ for $i \geq 1$. Let $z^{\text {red }}$ be a cycle in $G_{i}^{\text {red }}$. Let $z$ be a lifting of it to $G_{i}$. While $z$ does not need 
to be a cycle, we can choose $e^{\prime}$ such that $z^{q^{\prime}}$ is a cycle in $F^{e^{\prime}} G$. Since it is then in the tight closure of the boundaries, we obtain that for some $c \in R^{\circ}$ we have that $c\left(z^{q^{\prime}}\right)^{q} \in B^{\left[q^{\prime} q\right]}$ for all sufficiently large $q$, and this continues to hold when we tensor with $R_{\text {red }}$.

(c) The hypothesis of the phantom acyclicity criterion implies that the size $r_{i}$ minors generate an ideal of positive height and that the size $r_{i}+1$ minors are nilpotent. For large $e$ we then have that the size $r_{i}$ minors generate an ideal of positive height (and so are not all zero) while the size $r_{i}+1$ minors are zero, as required.

(d) The hypothesis that $c$ kills every $H_{i}\left(F^{e} G\right.$.) implies that if $z$ is a cycle in the $i$ th spot in any of these complexes, $F^{e} G$., then $c z^{q^{\prime}}$ is a boundary in $F^{e+e^{\prime}} G$. for all $q^{\prime}$ (respectively, for all sufficiently large $q^{\prime}$ ), which, by the right exactness of tensor, is exactly what we need to see that $z$ is in the tight closure of the module of boundaries in its complex. On the other hand, if all the $H_{i}\left(F^{e} G\right.$.) are phantom and $c$ is a test element, it is clear that $c$ kills all of them, since it kills $B_{G}^{*} / B$ for every pair of finitely generated modules $B \subseteq G$.

Proof of sufficiency in Theorem (9.8). By Lemma (9.15)(a), it suffices to show that $H_{i}\left(F^{e} G\right.$.) is phantom for sufficiently large $e, i \geq 1$. Replacing $G$. by $F^{e^{\prime}} G_{\text {. }}$, we may assume, by Lemma (9.15)(c), that rank $\alpha_{i}=r_{i}, 1 \leq i \leq n$, and that this continues to be true when we apply $F^{e}$.

Choose $c$ and $e^{\prime}$ as in Theorem (9.14). For each integer $e$, we let $q=p^{e}$ and apply Theorem $(9.13)$ to $F^{e}\left(G\right.$.) with $\phi=F^{e^{\prime}}$. Since applying $F^{e}$ replaces $I_{i}$ by $I_{i}^{[q]}$ but does not change its height, we conclude from Theorems (9.13) and (9.14) that $c^{q^{\prime}\langle\square n\rangle} F^{\square n}$ kills $H_{i}\left(F^{e}(G).\right)$ for all $e$. Since $q^{\prime}\langle\square n\rangle$ and $\square n$ do not depend on $e$, it follows that $G$. has phantom homology for $i \geq 1$.

The proof of Theorem (9.13) depends on the following lemma.

(9.16) Lemma. Let $R=R_{0} \rightarrow R_{1} \rightarrow \cdots \rightarrow R_{n}$ be a chain of ring homomorphisms, and let $c_{i}$ be an element of $R_{i}, 1 \leq i \leq n$. Let $M$. be a nonnegative left complex of $R$-modules such that $c_{i}$ kills $\operatorname{Im}\left(H_{i}\left(R_{i-1} \otimes_{R} M.\right) \rightarrow H_{i}\left(R_{i} \otimes_{R} M.\right)\right)$ for $1 \leq i \leq n-1$. Let $\theta_{i}$ denote the map from $M$. to $R_{i} \otimes M$. induced by tensoring $M$. with the map $R \rightarrow R_{i}$. Let $P$. be a nonnegative left complex of projective $R$-modules. Note that elements of $R_{i}$ act on $R_{j}$-modules if $i \leq j$, since we can map them to $R_{j}$.

(a) Suppose that we are given a map $f$ from $H_{0}\left(P_{\text {. }}\right)$ to $H_{0}\left(M_{\text {. }}\right)$. Then $c_{n-1} c_{n-2} \cdots c_{1}\left(\theta_{n-1} \circ f\right)$ lifts to a map of complexes $P . \rightarrow R_{n-1} \otimes_{R} M$. through $P_{n}$.

(b) Suppose that we have two liftings $g$. and $g^{\prime}$ of $f$ to maps of complexes. Let $d_{i}$ be the differential $P_{i} \rightarrow P_{i-1}$, and let $e_{i}$ be the differential in $M$. or any complex obtained from it by tensoring. Then there exist maps $h_{i}: P_{i} \rightarrow$ $R_{n-1} \otimes_{R} M_{i+1},-1 \leq i \leq n$ (where $P_{-1}=H_{0}\left(P_{.}\right), M_{-1}=H_{0}\left(M_{.}\right)$) such that $h_{-1}=0$ and such that $c_{n} c_{n-1} \circ c_{1}\left(\theta_{n} \circ\left(g_{i}-g_{i}^{\prime}\right)\right)=e_{i+1} h_{i}+h_{i-1} d_{i}$ for $0 \leq i \leq n$. 
Proof. (a) The argument begins precisely as in the usual case, and we simply give the inductive step. We may assume that $f^{\prime}=c_{n-2} \cdots c_{1}\left(\theta_{n-2} \circ f\right)$ has been lifted to a map $g .: P . \rightarrow R_{n-2} \otimes M$. out to the $(n-1)$ st stage. Let $c=c_{n-1}$ and $w: R_{n-2} \otimes M_{.} \rightarrow R_{n-1} \otimes M_{\text {. }}$

$$
\begin{aligned}
& R_{n-1} \otimes M_{n} \stackrel{e}{\longrightarrow} R_{n-1} \otimes M_{n-1} \stackrel{e}{\longrightarrow} R_{n-1} \otimes M_{n-2} \stackrel{e}{\longrightarrow} \cdots
\end{aligned}
$$

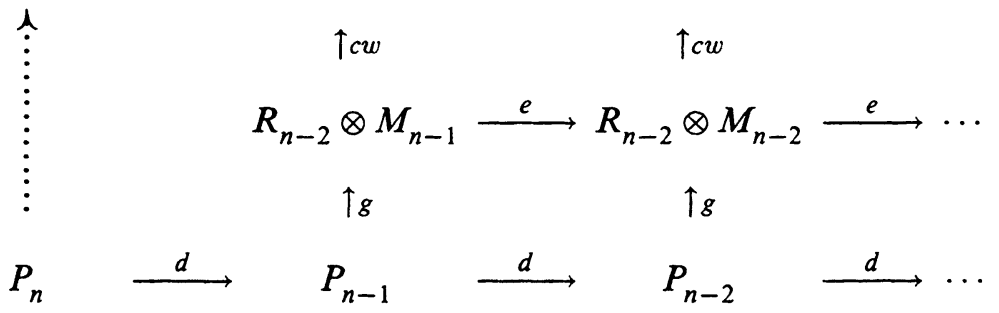

We must show that is it possible to fill in the map indicated by the dotted arrow in the diagram above so that it commutes. Since $d_{n-1} d_{n}$ is 0 , we know that $g_{n-2} d_{n-1} d_{n}=e_{n-1} g_{n-1} d_{n}=0$, and so $\operatorname{Im} g_{n-1} d_{n} \subseteq \operatorname{Ker} e_{n-1}$. Since $c w$ kills $H_{n-1}\left(R_{n-2} \otimes M_{\text {. }}\right)$, it follows that $c w g d\left(P_{n}\right) \subseteq e\left(R_{n-1} \otimes M_{n}\right)$, and so cwgd can be lifted to a map $P_{n} \rightarrow R_{n-1} \otimes M_{\text {. }}$, as required. This proves (a).

(b) By taking differences of maps, we may assume that $f$ and $g^{\prime}$ are 0 . We assume inductively that maps $h_{i}: P_{i} \rightarrow R_{n-1} \otimes M_{i+1}$ have been constructed out through $i=n-1$ with the required property. The early part of the argument is the same as in the standard construction of homotopies, and we assume that $n \geq 2$. Let $g^{\sim}$ denote $c_{n-1} \cdots c_{1} \theta_{n-1} g$. Let $w$ denote the map $R_{n-1} \otimes M$. $\rightarrow$ $R_{n} \otimes M$. . We replace the earlier $h_{i}$ by $h_{i}^{\prime}=c_{n} w h_{i}$, and it then suffices to show that one can fill in the dotted arrow in the diagram below with a map $h_{n}^{\prime}=h^{\prime}$ such that $c_{n} w g_{n}^{\sim}=c_{n} w h_{n-1} d_{n}+e_{n+1} h^{\prime}$, i.e., such that $e_{n+1} h^{\prime}=$ $c_{n} w\left(g_{n}^{\sim}-h_{n-1} d_{n}\right)$. Now,

$$
\begin{aligned}
e_{n}\left(g_{n}^{\sim}-h_{n-1} d_{n}\right) & =e_{n}{g_{n}^{\sim}}^{\sim}-e_{n} h_{n-1} d_{n} \\
& =g_{n-1}^{\sim} d_{n}-e_{n} h_{n-1} d_{n} \\
& =\left(g_{n-1}^{\sim}-e_{n} h_{n-1}\right) d_{n} \\
& =h_{n-2} d_{n-1} d_{n}=0,
\end{aligned}
$$

so that $\operatorname{Im}\left(g_{n}^{\sim}-h_{n-1} d_{n}\right) \subseteq \operatorname{Ker} e_{n}$.

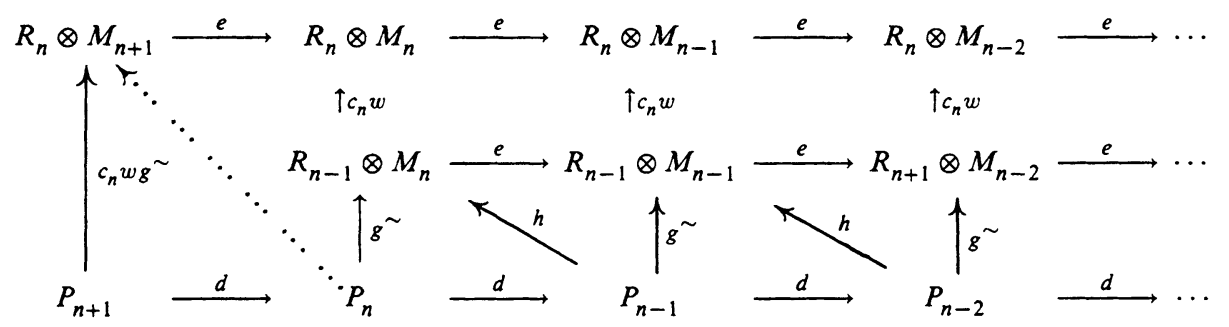

Since $c_{n} w$ kills $H_{n}\left(R_{n-1} \otimes M\right)$, it follows that $\operatorname{Im} c_{n} w\left(\tilde{g_{n}}-h_{n-1} d_{n}\right) \subseteq \operatorname{Im} e_{n+1}$, and this permits us to fill in the needed map. 
Remark. In the situation of Lemma (9.16)(b), if we are given some of the $h$ 's, say $h_{j}$ for $j<i$, the argument shows that we may construct $h_{i}, \ldots, h_{n}$ after multiplying the difference of the maps of complexes by operators that kill the homology at the $i$ th through $n$th spots.

Proof of Theorem (9.13). Let $\phi(c)=c^{q^{\prime}}$. When $n=0$, there are no values for $t$ in the specified range, and the result holds vacuously. We proceed by induction on $n$, assuming $n \geq 1$. We indicate the inductive step. Assume the result for complexes of length $n-1$ and consider a complex of length $n$. The conclusion we want, except at the $H_{1}$ spot, follows simply by considering the complex of length $n-1$ obtained by omitting $G_{0}$. Consequently, we consider a fixed cycle, represented by $z \in G_{1}$. We must show that $(c \Phi)^{\square(n-1)} z$ is a boundary. If this fails, we can preserve the failure after localizing at a prime. Our hypotheses all remain valid. It follows that we may assume that the ring is local.

Let $x_{1}, \ldots, x_{n}$ be a sequence in $I_{n}$ as in the definition of $(\phi, c)$-depth. Our hypotheses are stable under localization at $x_{i}$. If we localize at $x_{i}$, the minors of the last matrix in the resolution generate the unit ideal. We claim that when the maximal minors of the last matrix in $G$. generate the unit ideal, then $H_{n}(G)=$.0 while $(c \Phi)^{\square t}$ kills $H_{n-1-t}(G$. $)$ for $0 \leq t \leq n-2$. The statement for $H_{n}$ is well known. If the statement for $0 \leq t \leq n-2$ were false for some $t$, this could be preserved while localizing at a maximal ideal. However, once the ring is local, the fact that one of the maximal minors of the last matrix is a unit implies that, after a change of basis in $G_{n}$, we may assume that $G_{n}$ is generated by a subset of a free basis for $G_{n-1}$ and that the last map is an inclusion. The hypotheses are then valid for the complex

$$
0 \rightarrow G_{n-1} / G_{n} \rightarrow G_{n-2} \rightarrow \cdots \rightarrow G_{0},
$$

and the statement we want follows from the induction hypothesis applied to the displayed complex. We therefore conclude that $z^{\prime}=(c \Phi)^{\square(n-2)}[z]$ is a boundary after localization at $x_{i}$, which means that its homology class is killed by a power of $x_{i}$.

(At this point, we inject a comment about the case $n=1$. Let $z \in H_{1}\left(G_{\text {. }}\right) \subseteq$ $G_{1}$. Then some power of $x_{1}$, say $x_{1}^{w}$, kills $z$. But this means $z \in H_{1}\left(x_{1}^{w} ; G_{1}\right)$, a direct sum of copies of $H_{1}\left(x_{1}^{w} ; R\right)$, and so is killed by $c \Phi$. Henceforth, we assume that $n>1$.)

Since this is true for every $i$, we may choose $w$ such that $x_{1}^{w}, \ldots, x_{n}^{w}$ all kill the image $z^{\prime \prime}$ of $z^{\prime}\left(=(c \Phi)^{\square(n-2)}[z]\right)$ in the module

$$
M=\operatorname{Coker}\left(\Phi^{\square(n-2)} G_{2} \rightarrow \Phi^{\square(n-2)} G_{1}\right) .
$$

We change notation and write $x_{i}$ instead of $x_{i}^{w}$. Then there is a map from $R /\left(x_{1}, \ldots, x_{n}\right)$ to $M$ that sends the class of 1 to $z^{\prime \prime}$, which is induced by the map from $K_{0}(\mathbf{x} ; R)=R$ to $\Phi^{\square(n-2)} g_{1}$ that sends 1 to $z^{\prime}$. From Lemma (9.16)(a), we obtain a lifting of a certain "multiple" of this map to a map from the Koszul complex $K .(\mathbf{x} ; R)$ to a complex (truncated at the beginning) 
obtained from $\Phi^{\square(n-2)} G$. by further tensoring, using the fact that $(c \Phi)^{\square t}$ kills the homology of each complex $\Phi^{s} G$. at the $(n-t)$ th spot, $0 \leq t \leq n-2$. (We are using the fact that each of the complexes $\Phi^{s} G$. satisfies the same hypothesis as $G$. , and we are using the induction hypothesis repeatedly.)

To be precise, let $G_{j}^{+}=G_{j+1}$ if $j \geq 0, G_{j}=0$ if $j<0$, and let the differential on $G^{+}$be the same as on $G$. (except on $G_{0}$, which we have replaced by 0 in $\left.G_{.}^{+}\right)$. Then Lemma $(9.16)(a)$ yields a map from the Koszul complex $K .=K .(\mathbf{x} ; R)$ to $\Phi^{S}\left(\Phi^{\square(n-2)} G_{.}^{+}\right)$, where $S=\square(n-2)+\cdots+\square 0$, which, in degree 0 , sends 1 to $(c \Phi)^{S} z^{\prime}=(c \Phi)^{S+\square(n-2)} z$. We depict the situation in the diagram below.

Each number in the row of numbers at the top indicates what power of $c \Phi$ is needed to kill homology in $\Phi^{S} G$. at the spot directly beneath it. We have used a variable $s$ to remind the reader that $s$ varies in the proof of Lemma $(9.16)(a)$. The map we start with sends the generator 1 of $K_{0}$ to $(c \Phi)^{\square(n-2)} z$; each of the numbers in the row of exponents gets added during the course of construction of the vertical arrows, and the sum of these numbers is $S$.

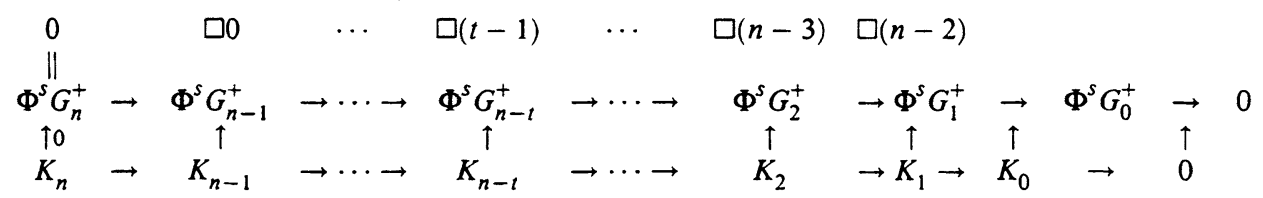

The map from $K_{n}$ is, of course, 0 , since $G_{n}^{+}=G_{n+1}=0$. This map from $K .(\mathbf{x} ; R)$ is $\phi^{S}$-linear, and we may replace $K .(\mathbf{x} ; R)$ by its tensor product with $\Phi^{S}(R)$, i.e., by $\Phi^{S} K .(\mathbf{x} ; R)=K$. $\left(\mathbf{x}^{S} ; R\right)$ to obtain an $R$-linear map.

Now apply $\operatorname{Hom}_{R}(, R)$. This produces a map $g$. from

$$
\operatorname{Hom}_{R}\left(\Phi^{S+\square(n-2)} G_{.}^{+}, R\right) \rightarrow \operatorname{Hom}_{R}\left(K .\left(\mathbf{x}^{S} ; R\right), R\right) .
$$

Note that the dual of the Koszul complex is a Koszul complex, and that the "first" map (to the dual of $\left.K_{n}\left(\mathbf{x}^{S} ; R\right)\right)$ is zero, since $G_{n+1}=0$. If we think of the numbering as reversed, we are in the situation of Lemma (9.16)(b). Recall that $c \Phi$ kills the homology of any Koszul complex on powers of the $x_{i}$ no matter what the exponent is!

We write $v$ to indicate $\operatorname{Hom}_{R}(, R)$. The situation is depicted in the diagram below. The row of 1's is a reminder that the exponent on $c \Phi$ needed to kill homology at the spot directly below in $K^{\vee}$ is always $1: c \Phi$ kills all such homology.

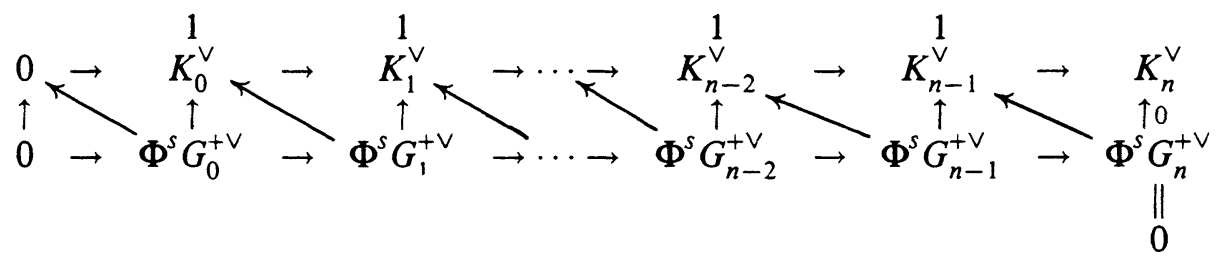

The exponent $s$ is $S+\square(n-2)$. We have two maps of complexes, $g$. and 0 ., inducing the same map on augmentations. We now apply Lemma $(9.16)(\mathrm{b})$ to 
construct diagonal arrows as indicated. We write $h_{t}$ for the map whose domain is $\Phi^{s} G_{n-t}^{+\vee}$. We obtain such $h_{t}$ after replacing $g$. by $(c \Phi)^{n} g$. Note that the powers of the $x$ 's in the Koszul complex have changed from $S$ to $S+n$ in the course of the argument. We now apply $\Phi^{n}$ to the bottom row so that we may assume that the vertical and slant arrows are $R$-linear. We continue to use $g$. and $h$. for the vertical and slant arrows: $g_{.}: \Phi^{s+n} G_{0}^{+\vee} \rightarrow K_{0}\left(\mathbf{x}^{S+n} ; R\right)$. Let $h=h_{n-1}$. Then $g_{0}=h d^{\vee}$. Dualizing again we obtain that $g_{0}^{\vee}=d h^{\vee}$. But $g_{0}^{\vee}(1)=(c \Phi)^{s+n} z$ is then the image of $d$, which proves that $(c \Phi)^{s+n}$ kills the typical cycle represented by $z$. Since we have

$$
\begin{aligned}
s+n & =\square(n-2)+S+n \\
& =\square(n-2)+(\square(n-2)+\cdots+\square 0)+n \\
& =\square(n-1),
\end{aligned}
$$

we can conclude that $(c \Phi)^{\square(n-1)}$ kills $H_{1}\left(G_{\text {. }}\right)$, completing the inductive step.

We need one more fact before proving the necessity part of Theorem (9.8).

(9.17) Proposition. Let $G$. be a possibly infinite complex of finitely generated projective modules over a Noetherian ring $R$ of characteristic $p$. Suppose that $M=\operatorname{Coker}\left(G_{h+1} \rightarrow G_{h}\right)$ is projective and that $H_{i}\left(G_{\text {. }}\right)$ is phantom for $i \geq h$. Then the complex

$$
\cdots \rightarrow G_{i} \rightarrow \cdots \rightarrow G_{h+1} \rightarrow G_{h} \rightarrow M \rightarrow 0
$$

is split exact.

Proof. Since the modules are projective, it suffices to prove exactness, and it will, in fact, suffice to prove exactness at $G_{h+1}$, for the hypotheses are stable and exactness at each successive point will then follow. Since $M$ is projective, $0 \rightarrow \operatorname{Im} G_{h+1} \rightarrow G_{h} \rightarrow M \rightarrow 0$ is split exact, and so $P=\operatorname{Im} G_{h+1}$ is projective. Thus, $0 \rightarrow Z \rightarrow G_{h+1} \rightarrow P \rightarrow 0$ is split exact, where $Z=\operatorname{Ker}\left(G_{h+1} \rightarrow G_{h}\right)$, and we can express $G_{h+1} \cong Z \oplus P$. Since $Z$ is in the tight closure of $B=$ $\operatorname{Im}\left(G_{h+2} \rightarrow G_{h+1}\right)$ in $G_{h+1} \simeq Z \oplus P$, we have that $Z$ is in the tight closure of $B$ in $Z$, by Proposition $(8.5)(\mathrm{m})$. But this implies that $Z=B$. (Otherwise, we can enlarge $B$ to $B^{\prime}$ so that $Z / B^{\prime} \cong R / m$ for a maximal ideal $m$. If $Z$ were in the tight closure of $B$, it would also be in the tight closure of $B^{\prime}$, and this would imply that $1 \in R$ is in the tight closure of $m$.)

Proof of necessity in Theorem (9.8). Now let $G$. be a fixed free complex, with notation as in (9.6), such that $H_{i}\left(F^{e} G\right.$.) is phantom for $i \geq 1$ and for all $e \geq 0$. First note that each $H_{i}\left(G_{\text {. }}\right)$, since it is phantom, is killed by an element of $R^{\circ}$. It follows that $G$. becomes acyclic if we tensor with $\left(R^{\circ}\right)^{-1} R_{\text {red }}$, which is isomorphic to the total quotient ring of $R_{\text {red }}$. It is then clear that the rank of $\alpha_{i}^{\mathrm{red}}$ is $r_{i}$, and that $I_{i}$ has positive height for $i \geq 1$. Assume there exist a ring $R$ and a complex for which the result fails, and choose $d$ as small as possible such that $I_{d}$ fails to have height at least $d$. By localizing at a minimal prime of $I_{d}$, we obtain an example in which $(R, m)$ is local and $\operatorname{dim} R<d$. The condition 
that every $H_{i}\left(F^{e} G_{.}\right)$is phantom is preserved by the localization. Likewise, we may replace $R$ by its completion and so assume that $R$ is complete. By Lemma (9.15)(b), we may replace $R$ by $R_{\text {red }}$ and thus assume also that $R$ is reduced. If one of the maps $G_{h+1} \rightarrow G_{h}$ has a free cokernel, then we may use Proposition (9.17) to split off the last part of the complex. Hence, using induction on the length of the complex, we may assume that all the $I_{h}$ are contained in $m$. By Corollary (6.26) there is a test element $c \in R^{\circ}$. In particular, $c$ kills the homology $H_{i}\left(F^{e}(G).\right)$ for all $e$ and all $i \geq 1$.

If $\operatorname{dim} R=0$, then $R$ is a field, phantom acyclicity implies acyclicity, and $I_{h} \subseteq m=0$ implies that $\alpha_{h}=0$, a contradiction if $d \geq 1$. If $\operatorname{dim} R=1$, then $R$ is C-M. If $d$ is at least 2 , then the last three terms of $G$. also give a counterexample. We might as well assume that $d=2$ is the length of $G$. Since ht $I_{2} \geq 1, I_{2}$ contains a nonzerodivisor and $\alpha_{2}$ is injective. By splitting off a summand of $G_{2}$ if necessary, we can also assume that $\alpha_{2}\left(G_{2}\right) \subseteq m G_{1}$, and hence that $\left(F^{e} \alpha_{2}\right)\left(G_{2}\right) \subseteq m^{[q]} G_{1}$. We can choose $e$ so large that $m^{[q]} \subseteq c^{2} R$, since $c$ is a parameter for $R$. Let $b$ be an element of the free basis for $G_{2}$. The $b^{q}$ is part of a free basis for $F^{e} G_{2}$ and, if $\beta=F^{e} \alpha_{2}$, we can write $\beta\left(b^{q}\right)=c^{2} w$ for some $w \in F^{e} G_{1}$. We shall obtain a contradiction by proving that $w$ represents an element of $H_{1}\left(F^{e} G\right.$.) not killed by $c$. Since $c$ is not a zerodivisor on the free module $F^{e} G_{0}$ and $c^{2}\left(F^{e} \alpha_{1}\right)(w)=\left(F^{e} \alpha_{1}\right)\left(c^{2} w\right)=$ $\left(F^{e} \alpha_{1}\right)\left(F^{e} \alpha_{2}\right)(b)=0$, we have that $\left(F^{e} \alpha_{1}\right)(w)=0$, so that $w$ does, in fact, represent an element of $H_{1}\left(F^{e} G\right.$.). To complete the argument, it suffices to show that $c w$ is not a boundary. Suppose we had $c w=\beta(g)$ for $g \in F^{e} G_{2}$. Then $c^{2} w=\beta(c g)=\beta(b)$, and, as already noted, $\beta$ is one-to-one, whence $b=c g$, a contradiction, since $c \in m$ and $b$ is a minimal generator of $F^{e} G_{2}$.

Now suppose that $\operatorname{dim} R \geq 2$. We can choose $x \in m \cap R^{\circ}$ such that the ideal $(c, x) R$ has height two. Let $R^{\prime}=R / x R$. We shall show that if $G^{\prime}$. is the complex obtained from $G$. by tensoring with $R^{\prime}$ and replacing the $G_{0} \otimes R^{\prime}$ term by 0 , numbered so that $G_{j}^{\prime}=G_{j+1} \otimes R^{\prime}$, then, working over $R^{\prime}, H_{j}\left(F^{e} G_{\text {. }}^{\prime}\right)$ is phantom for $j \geq 1$. This will contradict the minimality of $d$, since $\operatorname{dim} R^{\prime}<$ $d-1$ and $I_{d-1}$ for the complex $G^{\prime}$. is contained in $m R^{\prime}$. By Lemma $(9.15)(\mathrm{d})$, it will suffice to show that $c^{2}$ kills $H_{j}\left(F^{e} G^{\prime}\right.$ ) (with $F^{e}$ calculated over $R^{\prime}$ ) for all $e$, for the fact that $(x, c) R$ has height two implies that the image of $c$ in $R^{\prime}$ is in $R^{\prime \circ}$. But $H_{j}\left(F^{e} G_{.}^{\prime}\right) \cong H_{j+1}\left(F^{e} G_{.} \otimes R^{\prime}\right)$ for $j \geq 1$, and the short exact sequence $0 \rightarrow R \stackrel{x}{\rightarrow} R \rightarrow R^{\prime} \rightarrow 0$, tensored with $F^{e} G$., yields a long exact sequence for homology that includes

$$
\cdots \rightarrow H_{j+1}\left(F^{e} G_{.}\right) \rightarrow H_{j+1}\left(F^{e} G_{.} \otimes R^{\prime}\right) \rightarrow H_{j}\left(F^{e} G_{.}\right) \rightarrow \cdots
$$

Since $c$ (a test element) kills the first and last of the three terms shown, $c^{2}$ kills the middle term for $j \geq 1$.

We conclude this section with two examples.

(9.18) Example To see the need for calculating ranks in $R_{\text {red }}$ rather than $R$ in the statement of the phantom acyclicity criterion, consider the following 
example. Let $K$ be a field of characteristic $p$, and let

$$
R=K[S, T, U, V, X, Y] / J,
$$

where $J$ is generated by $U S+V T, X S+Y T$, and $(U Y-V X)^{t}$, where $t$ is an integer $\geq 2$. Let $\alpha$ have matrix $\left[\begin{array}{l}s \\ t\end{array}\right]$ and let $\beta$ have matrix $\left[\begin{array}{ll}u & v \\ x & y\end{array}\right]$, where the lower case letters denote the images of the corresponding capital letters. Then

$$
0 \rightarrow R \stackrel{\alpha}{\rightarrow} R^{2} \stackrel{\beta}{\rightarrow} R^{2} \rightarrow 0
$$

satisfies the phantom acyclicity criterion, and so this complex has phantom homology for $i \geq 1$, but $\beta$ has the wrong rank (rank 2) if we compute over $R$ instead of $R_{\text {red }}$.

(9.19) Example. Let $(R, m)$ be a C-M local ring of characteristic $p$ with $\operatorname{dim} R=n$. Let $x_{1}, \ldots, x_{n}$ be a system of parameters and $y \in m$ be such that if $I=\left(x_{1}, \ldots, x_{n}\right) R$, then $I:{ }_{R} y R \subseteq I^{*}$. We shall show below that there exist many examples of this type, even with $R$ a local isolated hypersurface singularity. In this situation, the Koszul complex $K .\left(x_{1}, \ldots, x_{n}, y ; R\right)$ has phantom homology for $i \geq 1$, but this is not preserved when we apply $F^{e}$. Indeed, it cannot be, since the complex is too long to satisfy the phantom acyclicity criterion. The Koszul homology here is 0 for $i \geq 2$ since the $x_{i}$ form a regular sequence. To see that $H_{i}$ is phantom, we must show that any relation $\rho=\left(r_{1}, \ldots, r_{n}, s\right)$ on $x_{1}, \ldots, x_{n}, y$ is in the tight closure of the trivial (or Koszul) relations. Since $s \in I: y R \subseteq I^{*}$, we can choose $c$ such that $c s^{q} \in I^{[q]}$ for all $q \gg 0$. It follows that we can subtract a linear combination of $q$ th powers of trivial relations from $\rho^{q}$ so as to make the last entry 0 . What remains is, essentially, a relation on the elements $x_{1}^{q}, \ldots, x_{n}^{q}$, and is thus a linear combination of trivial relations on these. But each such trivial relation is a $q$ th power of a trivial relation on $x_{1}, \ldots, x_{n}$.

Specifically, let $S$ be either $K\left[X_{1}, \ldots, X_{n}, Y\right]_{m}$, where $m$ is the homogeneous maximal ideal, or $K\left[\left[X_{1}, \ldots, X_{n}, Y\right]\right]$, let $R$ be the local hypersurface $S / H S$, where

$$
H=Y^{2}-\left(X_{1}^{d_{1}}+\cdots+X_{n}^{d_{n}}\right),
$$

and let $y, x_{i}$ be the images of $Y, X_{i}, 1 \leq i \leq N$. As above, let $I=$ $\left(x_{1}, \ldots, x_{n}\right) R$. For any sufficiently large choices of the $d_{i}, y$ will be in $I^{*}$ in $R$ for all but finitely many choices of the characteristic of $K$. This follows from the results of [HH5] on Gorenstein rings (a Gorenstein local ring is Fregular if and only if the ideal generated by one system of parameters is tightly closed) and [HH7] on rational singularities (if $R$ has isolated singularities and is F-regular, then the $\operatorname{Spec} R$ must have rational singularities). Over a field of characteristic 0 , the hypersurface will be an isolated but not rational singularity, and since it is Gorenstein, it will follow that $I$ is not tightly closed for almost all choices of the characteristic. But $y$ generates the socle modulo $I$, and so must be in $I^{*}$ if $I^{*} \neq I$. Note that $I:{ }_{R} y=m_{R}=I+y R$, and so $I:{ }_{R} y=m_{R} \subseteq I^{*}$, since $y \in I^{*}$, and this is what we needed. (The results of $\S 5$ on the Briançon-Skoda theorem can also be used to show that $y \in I^{*}$ if the $d_{i}$ 
are sufficiently large, independent of the characteristic. E.g., if all the $d_{i} \geq 2 n$, then $y^{2} \in I^{2 n} \Rightarrow y \in\left(I^{n}\right)^{-} \subseteq I^{*}$ by Theorem (5.4).)

\section{THE SYZYGY THEOREM AND VARIANT NOTIONS OF TIGHT CLOSURE IN CHARACTERISTIC $p$}

In this section we introduce, for Noetherian rings of characteristic $p$, a family of notions of tight closure. Our original notion is one of them, the one we feel is best "for general purposes." However, the others will clearly be of some importance. We shall use one of them, later in this section, to give a direct proof of the syzygy theorem in characteristic $p$. Another of these notions is explored in some detail in the next section. It strengthens the conclusions one can derive from the phantom acyclicity criterion, and plays a key role in the proof that direct summands of regular rings containing a field are CohenMacaulay (a result that is obtained in [HH7] in complete generality for the first time).

(10.1) Definition. Let $R$ be a Noetherian ring of characteristic $p$, and let $\mathscr{C}$ be a nonempty family of ideals of $R$ directed by $\supseteq$, i.e., for all $C, C^{\prime} \in \mathscr{C}$, there is an ideal $C^{\prime \prime} \subseteq C \cap C^{\prime}$ such that $C^{\prime \prime} \in \mathscr{C}$. If $N \subseteq M$ are $R$-modules, we say that an element $x \in M$ is in the tight closure of $N$ in $M$ with respect to $\mathscr{C}$, denoted $\mathscr{C}_{M} N_{M}^{*}$ or $\mathrm{Cl}_{\mathscr{C}}(N, M)$, if there exists an ideal $C \in \mathscr{C}$ such that for all $q \gg 0, C x^{q} \in N^{[q]} \subseteq F^{e}(M)$.

We shall say that $x \in M$ is in the small tight closure of $N$ in $M$ with respect to $\mathscr{C}$, denoted $\mathscr{C}_{M} N_{M}^{* s}$, if there exists an ideal $C \in \mathscr{C}$ such that $C x^{q} \in N^{[q]}$ for all $q$.

We shall say that a complex of modules $M^{\prime} \rightarrow M \rightarrow M^{\prime \prime}$ has phantom homology with respect to $\mathscr{C}$ at $M$ (or that the homology at $M$ is $\mathscr{C}$-phantom, or that the homology at $M$ is phantom in the sense of tight closure with respect to $\mathscr{C}$ ) if the cycles in $M$ are in the tight closure, in $M$, of the boundaries with respect to the family $\mathscr{C}$.

(10.2) Discussion and examples. We omit the subscripts $M$ and $\mathscr{C}$ whenever they are clear from context. Note that we could allow arbitrary subsets of $R$ in $\mathscr{C}$ instead of ideals, but that we get the same notion. We may replace each of these sets by the ideal it generates. For any given choice of $\mathscr{C}, N^{* s} \subseteq N^{*}$, of course.

Our standard notion of tight closure is the one obtained by letting $\mathscr{C}=$ $\left\{c R: c \in R^{\circ}\right\}$. Note that when $R$ has a test element $c$, we get the same notion by letting $\mathscr{C}=\{c R\}$ or by letting $\mathscr{C}=\{\tau(R)\} \quad(\tau(R)$ is the ideal generated by all the test elements). When $R$ is reduced or has a test element, the notions of tight closure and small tight closure coincide for $\mathscr{C}=\left\{c R: c \in R^{\circ}\right\}$. When the tight closure and the small tight closure are different, it is often problematic to decide which is the "better" notion.

We shall see in [HH5] that, if $R$ is reduced and $R^{1 / p}$ is module-finite over $R$, we also get the standard notion of tight closure by letting $\mathscr{C}$ be the set of powers of any ideal defining the nonregular locus in $\operatorname{Spec}(R)$. The key point is 
that for such an $R$, every element $c$ such that $R_{c}$ is regular has a power that is a test element. See also [HH3].

The condition that the family $\mathscr{C}$ be directed is needed to ensure that the tight closure is closed under addition.

If $\mathscr{C}=\{R\}$, the small tight closure of $N$ is $N$ itself, while the tight closure is the Frobenius closure of the submodule, i.e., the elements that are in the contraction of its expansion with respect to some power of the Frobenius endomorphism.

If $\mathscr{C}=\{(0)\}$, the tight closure of $N$ in $M$ is always $M$. Because this notion is completely uninteresting, one tends to feel that one ought to place a nondegeneracy condition on $c$ in the "all-purpose" notion of tight closure. However, it turns out that even when $c$ is nilpotent (and, perhaps, very badly a zerodivisor in other ways), the notion of tight closure with respect to $\{c R\}$ yields information. This point will be illuminated by the proof of the syzygy theorem below. It depends on the following.

(10.3) Lemma. Let $N \subseteq M$ be finitely generated modules over a local ring $(R, m)$, and let $\mathscr{C}$ be a family of nonzero ideals of $R$ directed by $\supseteq$. Then ${ }_{\mathscr{C}} N_{M}^{*} \subseteq N+m M$. Thus, if $x$ represents a minimal generator of $M / N$, then $x \notin \mathscr{C} N_{M}^{*}$.

Proof. We may kill $N$ without affecting any relevant issue. It suffices then to show that $m M$ is tightly closed in $M$ with respect to $\mathscr{C}$, and we may then kill $m M$. In this way, we reduce the problem to showing that 0 is tightly closed in $M / m M$, which is a finite direct sum of copies of $K$, and so it suffices to show that $m$ is tightly closed in $R$ with respect to $\mathscr{C}$. But if not, we would have $C 1^{q} \subseteq m^{[q]}$ for all $q \gg 0$ for a fixed nonzero ideal $C$ of $R$, a contradiction.

Before using this fact to prove the syzygy theorem, however, we want to discuss one more very important example of a variant notion of tight closure.

(10.4) Definition. Let $R$ be a Noetherian ring of characteristic $p$, and suppose that $Z=\left\{P \in \operatorname{Spec}(R): R_{P}\right.$ is not C-M $\}$ is closed. Let

$$
J=\bigcap\{P: P \in Z\}=\left\{c \in R: R_{c} \text { is C-M }\right\},
$$

the radical ideal defining the non-Cohen-Macaulay locus in $\operatorname{Spec}(R)$. Let $\mathscr{C}$ be the family $\left\{J^{t}: t\right.$ is a positive integer $\}$. We refer to $\mathscr{C}_{M}^{*}$ (respectively, $\mathscr{C}_{M} N_{M}^{* s}$ ) as the Cohen-Macaulay tight closure or C-M tight closure of $M$ in $N$ (respectively, the small C-M tight closure), and denote it $N_{M}^{* c m}$ (respectively, $N_{M}^{* c m s}$ ). As usual, the subscript $M$ is omitted whenever possible.

(10.5) Proposition. (a) With notation as in Definition (10.4), $N^{* c m s} \subseteq N^{* c m} \subseteq$ $N^{*}$.

(b) If $R$ is Cohen-Macaulay, $N^{* c m s}=N$ while $N^{* c m}$ is the Frobenius closure.

Proof. (a) The first inclusion is trivial, while the second follows from the fact that $J$ (and hence every $J^{t}$ ) meets $R^{\circ}$. $J$ cannot be contained in a minimal prime, since 0 -dimensional rings are $\mathrm{C}-\mathrm{M}$. 
(b) follows at once from the fact that when $R$ is C-M, $J$ is the unit ideal.

(10.6) Remarks. There are Cohen-Macaulay rings of characteristic $p$ such that every submodule of every module is contracted with respect to the Frobenius endomorphism, but which are not weakly F-regular. $K[X, Y] /(X Y)$ is an example. Thus, in general, $N^{* c m}$ is smaller than $N^{*}$. However, we shall show in the next section that, under mild restrictions on $R$, when a complex $G$. satisfies the phantom acyclicity criterion, the cycles are in the C-M tight closure of the boundaries. It turns out to be important to know this for the following reason. Suppose we map $R$ to $S$, where $S$ is regular, in such a way that for some $c \in R$ such that $R_{c}$ is C-M, the image of $c$ is not zero. (This condition is enormously weaker than requiring that $R \rightarrow S$ be injective!) Because a power of $c$ can be used as a "test element" for C-M tight closure, it follows that the map $H_{i}(G.) \rightarrow H_{i}(G . \otimes S)$ is $0, i \geq 1$. This is one of our main motivations for considering alternative notions of tight closure. The issue is discussed in detail in the next section.

Later we shall introduce the notion of weakly C-M rings and modules. See [HH4]. When the ring is not locally equidimensional, one gets corresponding results for the family $\left\{\mathscr{A}^{t}: t\right\}$, where $\mathscr{A}$ is the defining ideal of the nonweakly C-M locus in $R$, at least if $R$ is a homomorphic image of a Gorenstein ring of finite Krull dimension. We have not introduced special names for this kind of tight closure.

We now turn to the proof of the syzygy theorem mentioned earlier. First recall that if $x$ is an element of $M$, where $M$ is an $R$-module, the order ideal, $O_{M}(x)$, of $x$ is defined as $\left\{f(x): f \in \operatorname{Hom}_{R}(M, R)\right\}$. Although the syzygy theorem of Evans and Griffith (see [EvG1, EvG2]) ostensibly deals with the ranks of modules of syzygies, the heart of their result is really the following.

(10.7) Theorem (Evans-Griffith). Let $R$ be a local ring that contains a field. Let $M$ be a finitely generated $k$ th syzygy of finite projective dimension, and let $x$ be a minimal generator of $M$. Then the order ideal $O_{M}(x)$ has height at least $k$.

The condition that $M$ be a finitely generated $k$ th syzygy is equivalent to the existence of an exact sequence

$$
0 \rightarrow M \rightarrow G_{k-1} \rightarrow \cdots \rightarrow G_{i} \stackrel{\phi_{i}}{\rightarrow} G_{i-1} \rightarrow \cdots \rightarrow G_{0},
$$

where the $G_{i}$ are finitely generated free modules. We give an improved version of this result in which we show that the depth of $R$ on $O_{M}(x)$ is at least $k$. The proof, in characteristic $p$, is extremely simple. The general equicharacteristic case then follows by standard techniques of reduction to characteristic $p$, which are discussed in [HH7]. Explicitly,

(10.8) Theorem. Let $(R, m)$ be a local ring of characteristic $p$. Let $M$ be a finitely generated kth syzygy of finite projective dimension, and let $x$ be a minimal generator of $M$. Then the depth of $R$ on the order ideal $O_{M}(x)$ is at least $k$. 
Proof. We may think of $M$ as $\operatorname{Ker} \phi_{k-1}$ in a free resolution of a module $N$. If the result is false, we can choose a maximal regular sequence $y_{1}, \ldots, y_{d}$ in $I=O_{M}(x)$ with $d<k$ and $c \in R-J$, where $J=\left(y_{1}, \ldots, y_{d}\right) R$, such that $c I \subseteq J$. Since $x$ is a minimal generator of $M$, we can choose a minimal generator $g$ of $G=G_{k}$ that maps to $x$ ( $G$ maps onto $\left.M\right)$. Let $B=\operatorname{Ker} \phi$, where $\phi=\phi_{k}$, so that $M \cong G / B$. Let $R^{\prime}=R / J, G^{\prime}=G . \otimes_{R} R / J$, and let $B^{\prime}$ be the image of $B$ in $G / J G=G^{\prime}$. We shall show that the image $g^{\prime}$ of $g$ in $G^{\prime}$ is in the tight closure of $B^{\prime}$ with respect to $\left\{c R^{\prime}\right\}$ over $R^{\prime}$. The point is that, since $\operatorname{pd}_{R} N$ is finite, $F^{e}$ is exact when applied to $G$. . Now $\operatorname{Tor}_{k}^{R}\left(R / J, F^{e} N\right)=0$ (because pd $R / J=d<k$ ), and so $H_{k}\left(F^{e} G . \otimes R^{\prime}\right)=0$, and $F^{e} G . \otimes R^{\prime}$ may be identified with $F^{e}\left(G_{.}^{\prime}\right)$ computed thinking over $R^{\prime}$. The definition of $I$ implies that every map from $M$ to a free module $H$ maps $x$ into $I H$. Since $\phi$ factors through $M$ and sends $g$ to $x, \phi(g) \subseteq I G_{k-1}$ and $F^{e} \phi\left(g^{q}\right) \subseteq I^{[q]} F^{e} G_{k-1}$. Working modulo $J$, we obtain that

$$
\begin{aligned}
F^{e} \phi^{\prime}\left(c g^{\prime[q]}\right) & =c F^{e} \phi^{\prime}\left(g^{\prime[q]}\right) \\
& \subseteq c I^{[q]} F^{e} G_{k-1}^{\prime} \\
& \subseteq c I F^{e} G_{k-1}^{\prime} \\
& \subseteq J F^{e} G_{k-1}^{\prime}=0,
\end{aligned}
$$

so that $c g^{\prime[q]}$ is a cycle, and the vanishing of $\operatorname{Tor}_{k}^{R}\left(R / J, F^{e} N\right)$ implies that $c g^{\prime[q]}$ is a boundary, i.e., is in $B^{\prime q]}$ (using, tacitly, the right exactness of tensor). This is exactly what we needed. Since $g^{\prime}$ is in the tight closure of $B^{\prime}$ in $G^{\prime}$, its image in $G^{\prime} / B^{\prime} \cong M / J M$ is in the tight closure of 0 in $M / J M$. But the image of $g^{\prime}$ in $M / J M$ is the same as the image of $x$, and so is a minimal generator for $M / J M$. But then Lemma (10.3) implies that the image of $g^{\prime}$ cannot be in the tight closure of 0 . This contradiction establishes the theorem.

It is not difficult to present a version of the above argument in which any explicit reference to notions of tight closure is suppressed. See [EvG3]. However, we feel that the above argument gives a certain kind of insight into what is happening that is lost any other way. Notice that, except possibly in the case $I=J$, the image of $c$ in $R / J$ is, in fact, a zerodivisor, since $c I \subseteq J$.

We conclude this section with several corollaries of the above result. We first note the following result, which does not appear to be available in exactly this form in the literature, although it is quite similar to results in $[\mathrm{AuBr}]$ and $[\mathrm{BrV}]$ and is readily deducible from a result explicit in [BrV].

(10.9) Lemma. Let $R$ be an arbitrary Noetherian ring, and let $M$ be a finitely generated $R$-module. Suppose $\operatorname{pd}_{R} M<\infty$. Let $k \in \mathbf{N}$. Then the following two conditions are equivalent.

(a) $M$ is a kth syzygy of a finitely generated $R$-module $N$.

(b) For every prime ideal $P$ of $R$, either $M_{P}$ is free or depth $M_{P} \geq k$. 
Proof. First assume (a). If $M_{P}$ is not free,

$$
\text { depth } \begin{aligned}
M_{P} & =\operatorname{depth} R_{P}-p d_{R_{P}} M_{P} \\
& =\operatorname{depth} R_{P}-\left(\operatorname{pd}_{R_{P}} N_{P}-k\right) \\
& =\operatorname{depth} N_{P}+k,
\end{aligned}
$$

by the Auslander-Buchsbaum theorem (applied twice).

Since (b) evidently implies the condition $\left(\widetilde{S}_{k}\right)$ defined in Proposition (16.29) on p. 213 of [BrV], it follows at once from Proposition (16.33) on p. 215 of $[\mathrm{BrV}]$ that $(\mathrm{b}) \Rightarrow(\mathrm{a})$.

We next observe the following consequence of Theorem (10.8).

(10.10) Corollary. If $x_{1}, \ldots, x_{t}$, where $1 \leq t \leq k$, are part of a minimal set of generators for a $k$ th syzygy $M$ of finite projective dimension over a local ring $R$ of characteristic $p$, then $F=R x_{1}+\cdots+R x_{t}$ is a free module with $x_{1}, \ldots, x_{t}$ as free basis, and $M / F$ is a $(k-t)$ th syzygy. Moreover, the ideal $J$ of $R$ such that $c \in J$ precisely if $0 \rightarrow F_{c} \rightarrow M_{c}$ splits has depth at least $k-t+1$. (If $t=1, J$ is the same, up to radicals, as the order ideal of $x=x_{1}$.)

Proof. If $t=1$, we know that the order ideal of $x=x_{1}$ has depth at least $k \geq 1$. Since $x$ can be mapped to a nonzerodivisor in $R, \operatorname{Ann}_{R} x=0$, and so $R x \cong R$. We must see that $M / x R$ is a $(k-1)$ th syzygy. But if we localize at a prime $P$ that contains the order ideal of $x$, then $\operatorname{depth} R_{P} \geq k$, and so $\operatorname{depth} M_{P} \geq k$ (since $M_{P}$ is a $k$ th syzygy), and then $\operatorname{depth}(M / R x)_{P} \geq$ $k-1$, while otherwise $(M / R x)_{P}$ is a direct summand of $M_{P}$ and so is free (respectively, has depth $\geq k$ ) if $M_{P}$ satisfies the condition. This shows that $M / R x$ is a $(k-1)$ th syzygy, as claimed.

Now assume that the result has been established for sequences of minimal generators of length less than $t$. Let $J_{i}$ be the order ideal of the image of $x_{i}$ in $M_{i}=M / F_{i}$, where $F_{i}=\sum_{j<i} R x_{j}$. From the induction hypothesis, $J_{1}, \ldots, J_{t-1}$ have depths at least $k, k-1, \ldots, k-t+2$, respectively, and $J_{t}$ has depth at least $k-t+1$ because the image $y$ of $x_{t}$ in $M_{t}$ is a minimal generator in a $(k-t+1)$ th syzygy of finite projective dimension. The fact that $R y \cong R$ shows that $F$ is free, and if we localize at any element in $J=\bigcap_{j=1}^{t} J_{j}$, we see easily that $F$ splits off from $M$ (by splitting off the images of the cyclic free modules generated by $R x_{1}, R x_{2}$, etc., one at a time). Moreover, depth $J=\min _{t}$ depth $J_{t} \geq k-t+1$. The result now follows from the case $t=1$ applied to the element $y$ in $M_{t}$.

Note that Coroilary (10.10) is a rather strong form of the original syzygy theorem. If $M$ has $\leq k$ minimal generators, it will be free, while otherwise it will have a free submodule of rank $k$ (generated by any $k$ element subset of a set of minimal generators).

We conclude this section with a matrix interpretation of Corollary (10.10).

(10.11) Corollary. Let $R$ be a Noetherian local ring of characteristic $p$, and let $G$. be a finite free resolution over $R$. Consider a matrix $\alpha$ for the map of free modules $G_{k} \rightarrow G_{k-1}$, let $\operatorname{Im} G_{k}=M \subseteq G_{k-1}$, and consider the matrix $\beta$ 
formed from any $t$ columns of $\alpha$ corresponding to elements $f_{1}, \ldots, f_{t}$ in the free basis for $G_{k}$ that map to part of a minimal set of generators of $M$, where $1 \leq t \leq k$. Then $I_{t}(\beta)$ has depth $\geq k-t+1$.

Proof. If not, we can localize at a prime containing $I_{t}(\beta)$ in such a way that the depth of the ring becomes $<k-t+1$. Since depth $R \leq k-1$ and Coker $\alpha$ is a $(k-1)$ th syzygy, Coker $\alpha$ is free, and so $M$ is a free summand of $G=G_{k-1}$. By Corollary (10.10), the submodule $F$ generated by the images of $f_{1}, \ldots, f_{t}$ will be a free summand of $M$. Thus, $F$ will be a free summand of $G$, which implies that $\operatorname{Coker} \beta$ is free of rank $r-t$. But a necessary and sufficient condition for Coker $\beta$ to be free of rank $r-t$ is that $I_{t}(\beta)$ be the unit ideal, a contradiction.

(10.12) Remark. Note that when $t=1, I_{1}(\beta)$, the ideal generated by the elements of a column corresponding to a minimal generator $x$ of $M$, is always contained in the order ideal of $x$. More generally, in the situation of Corollary (10.11), if $N=$ Coker $\alpha, \Lambda^{t} \alpha$ gives a presentation of $\Lambda^{t} N$, and $f_{1} \wedge \cdots \wedge f_{t}$ maps to a certain element $u$ in $\operatorname{Im}\left(\bigwedge^{t} \alpha\right)$ (which is a first module of syzygies for $\left.\Lambda^{t} M\right)$. The order ideal of $u$ in $\operatorname{Im}\left(\Lambda^{t} \alpha\right)$ contains $I_{t}(\beta)$ and so has depth at least $k-t+1$.

\section{Phantom acyclicity for images of Gorenstein Rings}

In this section, we are concerned with showing that under mild conditions on a Noetherian ring $R$, there exist fixed elements $c \in R^{\circ}$ that kill all higher Koszul homology on parameters. For example, when $R$ is a locally equidimensional image of a Gorenstein ring of finite Krull dimension, every element $c$ such that $R_{c}$ is Cohen-Macaulay has a power that has this property. Results of this type are not dependent on any assumptions about the characteristic. We then use the techniques of $\S 9$ to show that each such $c$ has a power that kills the higher homology of every complex that satisfies the phantom acyclicity criterion. In consequence, we are able to show that in such a complex the cycles are in the C-M tight closure of the boundaries (in the sense of $\S 10$ ). Roughly speaking, this gives us a larger class of elements that can be used as test elements.

The availability of elements of $R^{\circ}$ that kill all higher Koszul homology of parameters noticeably simplifies the arguments of $\S 9$. There, in order to obtain the results for images of arbitrary C-M rings, it was necessary to apply fixed powers of the Frobenius endomorphisms as well as multiplication by a fixed element of $R^{\circ}$ to kill such homology.

Several of the results of this section are close cousins of Theorem 1 of [Ro1]. Theorem (11.5) can easily be deduced from it. The arguments given here are a bit more elementary, in that the use of dualizing complexes and spectral sequences is avoided. In the excellent case, similar results can be obtained by using the idea that occurs in the proof of Theorem (4.8) of this paper (and in the proof of the existence of big C-M modules in [Ho3]). After reducing to the complete case, one can arrange that a power of the constant $c$ multiplies the ring being studied into a free module over a regular ring. These results are an important ingredient in the proof in [HH9] that the integral closure of 
a biequidimensional, excellent, semilocal domain of characteristic $p$ in an algebraic closure of its fraction field is a big Cohen-Macaulay module for the original domain.

Until the contrary is specified, after the statement of Theorem (11.8), there are no assumptions on the characteristics of the rings in this section. However, all given rings are assumed Noetherian.

Before beginning our systematic study of images of Gorenstein rings, we note one other situation in which we know that elements $c \in R^{\circ}$ as mentioned above exist. This result is simply a restatement of what was proved in Theorem (9.14)(b), with the language of " $c$-depth" suppressed.

(11.1) Proposition. Let $R$ be reduced, locally equidimensional, and a homomorphic image of a $C-M$ ring $S$, say $R=S / Q$. Suppose also that for each minimal prime q of $J, S_{q}$ is regular. If $\operatorname{Spec}(R)$ is connected, then there exists an ideal $J_{0} \subseteq J$ generated by a regular sequence and an element $c^{\prime} \in S$ not in any minimal prime of $J$ such that $c^{\prime} J \subseteq J_{0}$. If $c$ is the image of $c^{\prime}$ in $R$, then $c \in R^{\circ}$, and for every ideal $I$ of $R$ such that ht $I \geq n$, there exist $x_{1}, \ldots, x_{n} \in I$ such that $c$ annihilates $H_{i}\left(x_{1}^{t}, \ldots, x_{n}^{t} ; R\right)$ for all $i, t \geq 1$.

If $\operatorname{Spec}(R)$ is not necessarily connected, an element $c \in R^{\circ}$ can be constructed componentwise by carrying through the procedure above for each component separately, and it will again have the property that for every ideal $I$ of $R$ with ht $I \geq n$, there exist $x_{1}, \ldots, x_{n} \in I$ such that $c$ kills $H_{i}\left(x_{1}^{t}, \ldots, x_{n}^{t} ; R\right)$ for all $t \geq 1$ and all $i \geq 1$.

(11.2) Remarks. The statement above is a bit subtle. The $c$ we construct above kills all $H_{i}\left(x_{1}^{t}, \ldots, x_{n}^{t} ; R\right), i \geq 1, t \geq 0$, but not for every choice of the $x_{i}$ such that $\operatorname{ht}\left(x_{1}, \ldots, x_{n}\right) R=n$. Rather, given $I$ of height $n$, Proposition (11.1) guarantees that we can choose $x_{1}, \ldots, x_{n} \in I$ such that $c$ kills the $H_{i}\left(\mathbf{x}^{t} ; R\right)$ for all $i \geq 1, t \geq 0$. However, Theorem (9.13) guarantees that a fixed power of $c$ will kill all $H_{i}\left(x_{1}, \ldots, x_{n} ; R\right)$ for parameters $x_{i}$, at least if there is a bound on $n$, e.g., if Krull $\operatorname{dim} R$ is finite. First, we restate the special case of Theorem (9.13) where the endomorphism $\phi$ of $R$ is the identity (still avoiding the " $c$-depth" terminology of Definition (9.11)). Recall that the function $\square t$ is defined following Remarks (9.12).

(11.3) Theorem. Let $R$ be an arbitrary Noetherian ring. Let $G$. be a finite complex of finitely generated free $R$-modules, and let $b_{i}, r_{i}$ and $\alpha_{i}$ be as in (9.6). Suppose for $1 \leq i \leq n$ that $\operatorname{rank} \alpha_{i}=r_{i}$, and let $I_{i}=I_{r_{i}}(\alpha)$. Let $c \in R$, and suppose that for every $i, 1 \leq i \leq n$, for every $j, 1 \leq j \leq i$, there exist $x_{1}, \ldots, x_{j} \in I_{i}$ such that $c$ kills $H_{\nu}\left(x_{1}^{t}, \ldots, x_{j}^{t} ; R\right)$ for all $\nu, t \geq 1$. Then $c^{\square t}$ kills $H_{n-t}\left(G_{\text {. }}\right), 0 \leq t \leq n-1$.

Our primary goal in the remainder of this section is to develop other situations in which there is a fixed element $c \in R^{\circ}$ that kills all higher Koszul homology of parameters. Our main result along these lines is Theorem (11.5) below. 
(11.4) Theorem. Let $R$ be an arbitrary Noetherian ring.

(a) Suppose that $R$ is locally equidimensional and a homomorphic image of a Gorenstein ring of finite Krull dimension. Let $c$ be any element of $R$ such that $R_{c}$ is $C-M$. Then $c$ has a fixed power that kills $H_{Q}^{i}\left(R_{Q}\right)$ for all $i<\mathrm{ht} Q$ for all prime ideals $Q$ of $R$.

(b) Suppose that $R$ has finite Krull dimension $\leq d$. Let $c$ be an element of $R$ that kills $H_{Q}^{i}\left(R_{Q}\right)$ for all $i<\mathrm{ht} Q$ for every prime ideal $Q$ of $R$. Then for every sequence of elements $x_{1}, \ldots, x_{n}$ of $R$ such that $\mathrm{ht}\left(x_{1}, \ldots, x_{n}\right) R \geq n$, $c^{2^{d}-1}$ kills $H_{i}\left(x_{1}^{t}, \ldots, x_{n}^{t} ; R\right)$ for every $i \geq 1$ and for all $t \in \mathbf{N}$.

Postponing the proof for a moment, we first state the following immediate corollary.

(11.5) Theorem. Let $R$ be an arbitrary Noetherian ring. Suppose that $R$ is a homomorphic image of a Gorenstein ring of finite Krull dimension and is locally equidimensional. Suppose that $c \in R$ is an element such that $R_{c}$ is Cohen-Macaulay. Then there is a fixed power $c^{\prime}$ of $c$ such that for every sequence of elements $x_{1}, \ldots, x_{n}$ of $R$ such that $\mathrm{ht}\left(x_{1}, \ldots, x_{n}\right) R \geq n, c^{\prime}$ kills $H_{i}\left(x_{1}^{t}, \ldots, x_{n}^{t} ; R\right)$ for all $i, t \geq 1$.

(11.6) Remark. In both Theorems (11.4)(b) and (11.5), the hypothesis that $\operatorname{ht}\left(x_{1}, \ldots, x_{n}\right) R \geq n$ is preserved when we replace the $x$ 's by their $t$ th powers. Thus, we could have omitted any reference to $t$ in the statements of these theorems without actually weakening their conclusions. We chose to include $t$ to make it obvious that the hypothesis of Theorem (11.3) holds in later applications.

Proof of Theorem (11.4). (a) We may assume $\operatorname{Spec}(R)$ is connected, for we can construct the needed power working on each connected component separately, if necessary. It then follows that $R=S / J$, where $S$ is Gorenstein of finite Krull dimension and all minimal primes of $J$ have the same height, as in the proof of Theorem (7.9). Let $h$ be the height of $J$. We claim that there is a power of $c$ that kills each of the modules $\operatorname{Ext}_{S}^{j}(R, S), j \neq h$. To see this, note that it suffices to prove that these $R$-modules, which vanish for $j>\operatorname{dim} S$, and which are finitely generated, vanish after localizing at $c$, and hence to prove that they vanish after localizing at any prime $q$ of $S$ such that $c \notin q$ and $q \supseteq J$. Let $Q=q / J$. Then $R_{Q}=S_{q} / J S_{q}$ is Cohen-Macaulay, height $J S_{q}=h$ (since all minimal primes of $J$ have height $h$ ), and so $\operatorname{Ext}_{S_{q}}^{j}\left(R_{Q}, S_{q}\right)=0$ if $j \neq h$, as required.

Let $c^{\prime}$ be a power of $c$ that kills the modules $\operatorname{Ext}_{S}^{j}(R, S)$ for $j \neq h$. Then, given a prime $Q$ of $R$, we may think of it as $q / J$ for a certain prime $q$ of $S$, and localizing at $q$ we find that $c^{\prime}$ kills $\operatorname{Ext}_{S_{q}^{j}}\left(R_{Q}, S_{q}\right)$. By local duality over $S_{q}$ (see $\left.[\mathrm{GrH}]\right), H_{Q}^{i}\left(R_{Q}\right)$ is the dual, into the injective hull of $S_{q} / q S_{q}$ over $S_{q}$, of $\operatorname{Ext}_{S_{q}}^{j}\left(R_{Q}, S_{q}\right)$, where $j=\operatorname{dim} S_{q}-i$. It now follows that $c^{\prime}$ kills each 
$H_{Q}^{i}\left(R_{Q}\right)$ for $i<\mathrm{ht} Q$, since $i \neq \mathrm{ht} Q$ if and only if $j=\operatorname{dim} S_{q}-i \neq h$. This establishes (a).

(b) As observed in Remark (11.6), it suffices to consider the case $t=1$. Let $D=D(d)=2^{d}-1$. Suppose that $c^{D}$ fails to kill $H_{i}\left(x_{1}, \ldots, x_{n} ; R\right), i \geq 1$. Then this remains true for some localization of $R$. Thus, we may assume without loss of generality that $(R, m, K)$ is a local ring in the statement of the theorem. We may then assume that the $x_{i}$ are in $m$, since otherwise the Koszul homology vanishes. We use induction on $d$.

If $d=0$, then the sequence of $x$ 's must be empty, and $H_{i}$ will be 0 for $i \geq 1$. Henceforth, we assume $d \geq 1$. Let $N$ denote the ideal $H_{m}^{\circ}(R)=$ $\bigcup_{t} \mathrm{Ann}_{R} m^{t}$. Let $R^{\prime}=R / N$. Note that $c$ kills $N$, by assumption. It will suffice to show that $c^{D(d)-1}$ kills $H_{i}\left(x_{1}, \ldots, x_{n} ; R^{\prime}\right), i \geq 1$, by virtue of the long exact sequence for Koszul homology induced by the short exact sequence $0 \rightarrow N \rightarrow R \rightarrow R^{\prime} \rightarrow 0$,

$$
\cdots \rightarrow H_{i}(\mathbf{x} ; N) \rightarrow H_{i}(\mathbf{x} ; R) \rightarrow H_{i}\left(\mathbf{x} ; R^{\prime}\right) \rightarrow \cdots,
$$

for then $c$ kills the leftmost term and $c^{D(d)-1}$ kills the rightmost term, which implies that $c^{D(d)}$ kills the middle term. We change notation and write $R$ for $R^{\prime}$, assuming that depth $R \geq 1$. Let $C=c^{2 D(d-1)}$. Since $D(d)-1=2 D(d-1)$, what we must show is that $C$ kills $H_{i}(\mathbf{x} ; R)$ when depth $R \geq 1$.

Consider a nonzerodivisor $y$ in $m$. Let $T=R / y R$. The long exact sequence for local cohomology shows that for any prime ideal $Q$ of $T$, which we may write as $q / y R$ for a certain prime $q$ of $R$, we have

$$
\cdots \rightarrow H_{q}^{i}\left(R_{q}\right) \rightarrow H_{Q}^{i}\left(T_{Q}\right) \rightarrow H_{q}^{i+1}\left(R_{q}\right) \rightarrow \cdots,
$$

and if $i<\mathrm{ht} Q$, then both $i$ and $i+1$ are $<\mathrm{ht} q$. Thus, $c^{2}$ kills all the $H_{Q}^{i}\left(T_{Q}\right)$ for $i<\mathrm{ht} Q$, and $\operatorname{dim} T \leq d-1$. It follows from the induction hypothesis that $\left(c^{2}\right)^{D(d-1)}=C$ kills $H_{i}(\mathbf{z} ; R / y R)$ for $i \geq 1$ and any sequence $\mathrm{z}=z_{1}, \ldots, z_{j}$ in $R$ such that $\mathrm{ht}\left(z_{1}, \ldots, z_{j}\right)(R / y R) \geq j$.

We consider two cases. If $n<\operatorname{dim} R$, by prime avoidance we can extend $x_{1}, \ldots, x_{n}$ to a sequence $x_{1}, \ldots, x_{n}, y$ such that $y$ is not a zerodivisor in $R$ and $\operatorname{ht}\left(x_{1}, \ldots, x_{n}, y\right) R=n+1$. Now suppose that $C$ does not kill a certain element $w \in H_{i}(\mathbf{x} ; R)$. Represent $w$ by a Koszul cycle $v \in K_{i}(\mathbf{x} ; R)$. Let $T_{t}=R / y^{t} R$. For $t \gg 0$, the image of $C v$ in $K_{i}\left(\mathbf{x} ; T_{t}\right)$ will represent a nonzero element of $H_{i}\left(\mathbf{x} ; T_{t}\right)$. (Otherwise, if $B$ denotes the module of Koszul boundaries in $G=K_{i}(\mathbf{x} ; R)$, we would have $C v \in B+y^{t} G$ for all $t$, which contradicts the fact that $G / B$ is $m$-adically separated.) But this contradicts the discussion of the preceding paragraph, since $y^{t}$ is a nonzerodivisor in $R$ for all $t$ and $h t\left(x_{1}, \ldots, x_{n}, y\right)=n+1 \Rightarrow \operatorname{ht}\left(x_{1}, \ldots, x_{n}\right) T_{t}=n$.

The second case is the one where $\eta=\operatorname{dim} R$. In this case, we know the depth of $R$ on $\left(x_{1}, \ldots, x_{n}\right)=J$ is at least 1 , and we can pick a minimal generator $y$ for $J$ which is a nonzerodivisor in $R$ (this only requires avoiding $m J$ and a finite set of primes). We can extend $y$ to a minimal set of generators for $J$. If we use these new generators to calculate the Koszul homology, 
we get the same result. Therefore, there is no loss of generality in assuming that $y=x_{n}$ is a nonzerodivisor. In this situation, $H_{i}\left(x_{1}, \ldots, x_{n-1}, y ; R\right) \cong$ $H_{i}\left(x_{1}, \ldots, x_{n-1} ; R / y R\right)$. (This is easily seen if one thinks of the Koszul homology as a Tor as in [S, p. IV-6], and then uses the long exact sequence that is the degeneration of the spectral sequence for change of rings for Tor (cf. [S, $\mathrm{p}$. $\mathrm{V}-17$, second paragraph from the bottom of the page]). Alternatively, because the sequence $0 \rightarrow R \stackrel{y}{\rightarrow} R \rightarrow R / y R \rightarrow 0$ is exact, the homology of

$$
K .\left(x_{1}, \ldots, x_{n-1} ; R\right) \otimes_{R}(0 \rightarrow R \stackrel{y}{\rightarrow} R \rightarrow 0)=K .\left(x_{1}, \ldots, x_{n} ; R\right)
$$

equals that of

$$
K .\left(x_{1}, \ldots, x_{n-1} ; R\right) \otimes_{R} R / y R=K .\left(x_{1}, \ldots, x_{n-1} ; R / y R\right) .
$$

But then our earlier argument shows that $C$ kills the latter module and, hence, the former.

(11.7) Definition. In discussing complexes satisfying the phantom acyclicity criterion in $\S 9$, the emphasis was on rings of characteristic $p$. But the conditions used in the criterion, which we review below, make sense and were defined over an arbitrary Noetherian ring $R$. However, we need to be able to refer to complexes that satisfy the condition on ranks even if one does not kill the nilpotents. We recall the conventions of (9.7). If $G$. is a finite complex of finitely generated free $R$-modules with notation as in (9.6), we shall say that $G$. satisfies the standard rank and height conditions if the rank of $\alpha_{i}=r_{i}$, $1 \leq i \leq n$, and height $I_{i} \geq i, 1 \leq i \leq n$. With this terminology, $G$. satisfies the phantom acyclicity criterion iff $G . \otimes_{R} R_{\text {red }}$ satisfies the standard rank and height conditions over $R_{\text {red }}$.

Using this terminology, we can put Theorems (11.5) and (11.3) together to get

(11.8) Theorem. Let $R$ be an arbitrary Noetherian ring, not necessarily of characteristic $p$. Suppose that $R$ is a homomorphic image of a Gorenstein ring of finite Krull dimension and is locally equidimensional. Let $c$ be an element of $R$ such that $R_{c}$ is Cohen-Macaulay. Then there is a fixed power $c^{\prime}$ of $c$ such that for every finite complex $G$. satisfying the standard rank and height conditions, $c^{\prime}$ kills $H_{i}(G$.$) for all i \geq 1$.

Hence, if $\mathscr{A}$ is the defining ideal of the non-Cohen-Macaulay locus in $R$, there is a power $\mathscr{A}^{\prime}$ of $\mathscr{A}$ such that for every finite complex $G$. satisfying the standard rank and height conditions, $\mathscr{A}^{\prime}$ kills $H_{i}\left(G_{\text {. }}\right)$ for all $i \geq 1$.

(11.9) Remark. One can get more specific information by tracing through the ideas in the proof of Theorem (11.5). In the connected case, let $R=S / J$ with $S$ Gorenstein of finite Krull dimension. Let $d=\operatorname{dim} R$. Replace $c$ by a power $c^{\prime \prime}$ that kills all the $\operatorname{Ext}_{S}^{j}(R, S)$ for $j \neq$ ht $J$. Then $c^{\prime \prime\left(2^{d}-1\right)}$ kills the higher Koszul homology of any sequence of parameters, and $c^{\prime \prime\left(2^{d}-1\right) \square(d-1)}$ kills the higher homology of any complex $G$. satisfying the standard rank and height conditions. (Note that if the length $n$ of $G$. is $>d=\operatorname{dim} R$, we must 
have that $I_{i}$ is the unit ideal for $i>d$. After one splits off the part of the complex containing the terms for $i>d$, if there are such terms, the complex that remains, which gives rise to all the homology of the original complex, has length at most $d$, and so we can use the exponent $\square(d-1)$ on $c^{\prime \prime\left(2^{d}-1\right)}$.) Of course, once a power of each element of $\mathscr{A}$ kills all such homology, so does a power of $\mathscr{A}$.

For the remainder of this section we assume that given rings have characteristic $p$. What we have proved yields the following result at once.

(11.10) Theorem. Let $R$ be a Noetherian ring of characteristic $p$ that is a homomorphic image of a Gorenstein ring of finite Krull dimension and is locally equidimensional. Let $\mathscr{A}$ be the defining ideal of the non-Cohen-Macaulay locus in $R$. Then there is a power $\mathscr{A}^{\prime}$ of $\mathscr{A}$ (chosen as in Theorem (11.8)) such that the following conditions on any finite complex $G$. of finitely generated free $R$-modules, with notation as in (9.6), are equivalent.

(a) For all sufficiently large $e, F^{e} G$. has phantom homology for $i \geq 1$.

(b) For all $e, F^{e} G$. has phantom homology for $i \geq 1$.

(c) For some element $c \in R^{\circ}, c$ kills $H_{i}\left(F^{e} G\right.$.) for all sufficiently large $e$ and all $i \geq 1$.

(d) $G$. satisfies the phantom acyclicity criterion.

(e) $F^{e} G$. satisfies the standard rank and height conditions for all sufficiently large $e$.

(f) For some power $\mathscr{A}^{N}$ of $\mathscr{A}, \mathscr{A}^{N}$ kills $H_{i}\left(F^{e} G_{.}\right)$for all $e \gg 0$ and all $i \geq 1$.

(g) $\mathscr{A}^{\prime}$ kills $H_{i}\left(F^{e} G\right.$.) for all $e \gg 0$ and all $i \geq 1$.

(h) For all $i \geq 1$ and all sufficiently large $e$, the cycles in $F^{e} G_{i}$ are in the small $C-M$ tight closure of the boundaries.

(i) For all $i \geq 1$ and all $e$, the cycles in $F^{e} G_{i}$ are in the $C-M$ tight closure of the boundaries.

Moreover, if these equivalent conditions are satisfied, then $\mathscr{A}^{\prime}$ kills $H_{i}\left(F^{e} G_{.}\right), i \geq 1$, for all $e$ such that $F^{e} G$. satisfies the standard rank and height conditions. In all of the statements above that refer to sufficiently large $e$, the statement will hold when $e$ is so large that the standard rank condition holds for $F^{e} G$. . In particular, if the standard rank and height conditions hold for $G$. itself, then each statement referring to "all sufficiently large $e$ " is equivalent to the corresponding statement with "all $e$ " instead. Thus, if $R$ is reduced (so that $G$. satisfies the phantom acyclicity criterion iff it satisfies the standard rank and height conditions), then "all sufficiently large $e$ " can be replaced by "all $e$ " throughout to obtain a new set of equivalent statements.

Proof. The equivalence of (a), (b), (c), (d), and (e) follows from Lemma (9.15) and the phantom acyclicity criterion of $\S 9$. Note that once the standard rank and height conditions hold, they continue to hold. Fix $e^{\prime}$ so that they hold for $e \geq e^{\prime}$. Theorem (11.8) shows that for $e \geq e^{\prime}, \mathscr{A}^{\prime} H_{i}\left(F^{e} G_{0}\right)=0, i \geq 1$, and so we have that $(\mathrm{e}) \Rightarrow(\mathrm{g}) \Rightarrow$ (f) in a strong form that shows that $(\mathrm{g})$, (f) hold for $e \geq e^{\prime}$ when (e) does. But (f) $\Rightarrow$ (i) by the definition of C-M tight closure and the same idea as in the proof of Lemma (9.15), and (i) $\Rightarrow(b)$, 
which we already know to be equivalent to (e). The argument given justifies the two statements that follow the list of equivalent conditions, and the remaining statements are straightforward consequences of the first two.

(11.11) Discussion. The next two theorems assert that under mild conditions on the ring $R$, the "kind" of phantom homology that "arises" from the phantom acyclicity criterion is killed if one maps to a regular (or sufficiently good weakly F-regular) ring $S$, provided that some element $c$ such that $R_{c}$ is CohenMacaulay is mapped into $S^{\circ}$. This simply means, geometrically, that the image of every component of $\operatorname{Spec}(S)$ meets the Cohen-Macaulay locus in Spec $R$. Thus, the kind of phantom homology that arises from the phantom acyclicity criterion may be thought of as a consequence of the failure of the Cohen-Macaulay property in $R$. It is worth noting that this is not the only kind of phantom homology. Cohen-Macaulay rings are not, in general, weakly F-regular. We also note that, in mapping to $S$, the height conditions are lost. If they were retained, we would get the vanishing we want from the Buchsbaum-Eisenbud criterion, and we would not need to impose any F-regularity condition on $S$. Only the Cohen-Macaulay property would be needed. We would then get that the tensor product of the original complex with $S$ is acyclic, not just the more subtle result that the induced map of homology is 0 . Moreover, we would not need any restrictions on the characteristic. The Frobenius endomorphism would not come into it.

The power of the theory presented here is that there is no necessity to preserve heights under the map to $S$, only to keep one element $c$ such that $R_{c}$ is C-M from being "too degenerate" in $S$.

The vanishing theorems for maps of homology follow.

(11.12) Theorem. Let $G$. be a finite free complex of finitely generated free modules over a Noetherian ring $R$ of characteristic $p$, as in (9.6). Moreover, suppose that $R$ is a homomorphic image of a Gorenstein ring of finite Krull dimension, and is locally equidimensional. Suppose that $G$. satisfies the phantom acyclicity criterion. Let $c \in R$ be such that $R_{c}$ is Cohen-Macaulay, and let $S$ be a regular (or weakly F-regular ring). Suppose that $h: R \rightarrow S$ with $h(c) \in S^{\circ}$. Then the induced map $H_{i}\left(G_{.}\right) \rightarrow H_{i}\left(G . \otimes_{R} S\right)$ is 0 for $i \geq 1$, and the same is true for the map $H_{i}\left(G_{.} ; \rightarrow H_{i}\left(G^{\prime}\right)\right.$ for any complex $G^{\prime}$. of $S$-modules to which G. maps.

Proof. Consider a cycle $z \in G_{i}$. We can assume that $G_{.}^{\prime}=G . \otimes_{R} S$, since the map to $G^{\prime}$. will factor through $G . \otimes_{R} S$. From Theorem (11.10), we know that $c$ has a power $c^{\prime}$ such that $c^{\prime} z^{q} \in B^{[q]}$ for all sufficiently large $q$, and it follows that $h\left(c^{\prime}\right) h(z)^{[q]} \in B^{\prime[q]}$ in $F^{e}\left(G^{\prime}\right)$ for all $e \gg 0$, where $B^{\prime}$ is the module of boundaries in $G_{i}^{\prime}$. Since $h\left(c^{\prime}\right) \in S^{\circ}$, we have that $h(z) \in B^{\prime *}=B^{\prime}$, as required.

We can prove the same vanishing theorem under somewhat different hypotheses on $R$ and $S$.

(11.13) Theorem. Let $G$. be a finite free complex of finitely generated free modules over a Noetherian ring $R$ of characteristic $p$, as in (9.6). Moreover, 
suppose that $R$ has $C-M$ formal fibers and is locally formally equidimensional. Suppose that $G$. satisfies the phantom acyclicity criterion. Let $c \in R$ be such that $R_{c}$ is Cohen-Macaulay, and let $S$ be a regular ring (or a weakly F-regular ring with a completely stable test element). Suppose $h: R \rightarrow S$ with $h(c) \in S^{\circ}$. Then the induced map $H_{i}\left(G_{.}\right) \rightarrow H_{i}\left(G . \otimes_{R} S\right)$ is 0 for $i \geq 1$, and the same is true for the map $H_{i}\left(G_{0}\right) \rightarrow H_{i}\left(G_{\text {. }}^{\prime}\right)$ for any complex $G^{\prime}$. of $S$-modules to which G. maps.

Proof. If we have a counterexample, we can replace $S$ by its localization at a suitable maximal ideal, and we still have a counterexample. We can then replace $S$ by its completion. We can localize $R$ at the contraction of the maximal ideal of $S$ to $R$ without affecting any of our hypotheses and then complete. When we tensor $G$. with the completed, localized $R$, it still satisfies the phantom acyclicity criterion. Our hypotheses guarantee that the complete local ring $R^{\prime}$ is equidimensional. The fact that $R$ has C-M formal fibers implies that $R_{c}^{\prime}$ is C-M. But now $R^{\prime}$ satisfies the hypotheses of Theorem (11.12), and this gives a contradiction.

\section{ACKNOWLEGMENTS}

We would like to thank Jürgen Herzog and Joseph Lipman for helpful comments. We would also like to thank Ian Aberbach, Sankar Dutta, V. Kodiyalam, N. Mohan Kumar, K. N. Raghavan, Irena Swanson, and the referee for their comments and corrections.

Parts of this research were done at the Mathematical Sciences Research Institute, Berkeley and at the University of Stockholm. We would like to thank both for their hospitality.

\section{BIBLIOGRAPHY}

[A] M. Artin, Algebraic approximation of structures over complete local rings, Inst. Hautes Étude Sci. Publ. Math. 36 (1969), 23-56.

[AuBr] M. Auslander and M. Bridger, Stable module theory, Mem. Amer. Math. Soc., no. 94, Amer. Math. Soc., Providence, RI, 1969.

[B] J.-F. Boutot, Singularités rationelles et quotients par les groupes réductifs, Invent. Math. 88 (1987), 65-68.

[BE] D. Buchsbaum and D. Eisenbud, What makes a complex exact, J. Algebra 25 (1973), 259-268.

[Bor] A. Borel, Linear algebraic groups, Benjamin, New York, 1969.

[BrS] J. Briançon and $\mathrm{H}$. Skoda, Sur la clôture intégrale d'un idéal de germes de fonctions holomorphes en un point de $C^{n}$, C. R. Acad. Sci. Paris Sér. A 278 (1974), 949-951.

[BrV] W. Bruns and U. Vetter, Determinantal rings, Lecture Notes in Math., vol. 1327, SpringerVerlag, Berlin, 1988.

[DEP] C. De Concini, D. Eisenbud, and C. Procesi, Hodge algebras, Astérisque 91 (1982), 1-87.

[Du1] S. P. Dutta, Frobenius and multiplicities, J. Algebra 85 (1983), 424-448.

[Du2] _ On the canonical element conjecture, Trans. Amer. Math. Soc. 299 (1987), 803-811.

[Du3] _ Ext and Frobenius, J. Algebra (to appear).

[EHo] J. A. Eagon and M. Hochster, R-sequences and indeterminates, Quart. J. Math. Oxford Ser. (2) 25 (1974), 61-71. 
[Ei] D. Eisenbud, Homological algebra on a complete intersection with an application to group representations, Trans. Amer. Math. Soc. 260 (1980), 35-64.

[EvG1] E. G. Evans and P. Griffith, The syzygy problem, Ann. of Math. (2) 114 (1981), 323-333.

[EvG2] __ Syzygies, London Math. Soc. Lecture Note Ser., no. 106, Cambridge Univ. Press, Cambridge, 1985.

[EvG3] __, Order ideals, Commutative Algebra, Proc. Microprogram, June 15-July 12, 1987, Math. Sci. Res. Inst. Publ., no. 15, Springer-Verlag, New York, Berlin, Heidelberg, London, Paris, Tokyo, 1989, pp. 213-225.

[FeW] R. Fedder and $\mathrm{K}$. Watanabe, $A$ characterization of F-regularity in terms of F-purity, Commutative Algebra, Proc. Microprogram, June 15-July 12, 1987, Math. Sci. Res. Inst. Publ., no. 15, Springer-Verlag, New York, Berlin, Heidelberg, London, Paris, Tokyo, 1989, pp. 227-245.

[Fu] W. Fulton, Intersection theory, Springer-Verlag, Berlin, 1984.

[GraR] H. Grauert and O. Riemenschneider, Verschwindungsätze für analytische kohomologiegruppen auf komplexen Räumen, Invent. Math. 11 (1970), 263-290.

[GrH] A. Grothendieck (notes by R. Hartshorne), Local cohomology, Lecture Notes in Math., vol. 41, Springer-Verlag, Heidelberg, 1967.

[He] J. Herzog, Ringe der Charakteristik $p$ und Frobenius-funktoren, Math. Z. 140 (1974), 67-78. [HH1] M. Hochster and C. Huneke, Tightly closed ideals, Bull. Amer. Math. Soc. 18 (1988), 45-48.

[HH2] _ Tight closure, Commutative Algebra, Proc. Microprogram, June 15-July 12, 1987, Math. Sci. Res. Inst. Publ., no. 15, Springer-Verlag, New York, Berlin, Heidelberg, London, Paris, Tokyo, 1989, pp. 305-324.

[HH3] _ Tight closure and strong F-regularity, Mem. Soc. Math. France (N.S.), numéro consacré au colloque en l'honneur de P. Samuel (to appear).

[HH4] _ Phantom homology, preprint, 1989.

[HH5] _-, Tight closure, F-regularity, test elements and smooth base change (in preparation).

[HH6] __ Tight closures of parameter ideals and splitting in module-finite extensions (in preparation).

[HH7] _ Tight closure in characteristic zero (in preparation).

[HH8] _ Tight closure and elments of small order in integral extensions, preprint, 1989.

[HH9] __ Infinite integral extensions and big Cohen-Macaulay algebras, preprint, 1989.

[Ho1] M. Hochster, Rings of invariants of tori, Cohen-Macaulay rings generated by monomials, and polytopes, Ann. of Math. (2) 96 (1972), 318-337.

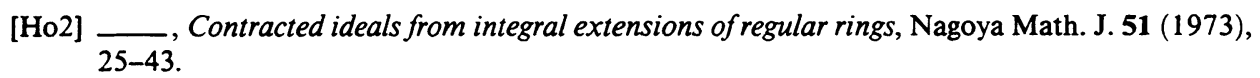

[Ho3] _ Topics in the homological theory of modules over commutative rings, C.B.M.S. Regional Conf. Ser. in Math., no. 24, Amer. Math. Soc., Providence, RI, 1975.

[Ho4] __, Big Cohen-Macaulay modules and algebras and embeddability in rings of Witt vectors, Proc. Queen's University Commutative Algebra Conference, Queen's Papers in Pure and Appl. Math. 42 (1975), 106-195.

[Ho5] _ Cyclic purity versus purity in excellent Noetherian rings, Trans. Amer. Math. Soc. 231 (1977), 463-488.

[Ho6] _ Some applications of the Frobenius in characteristic 0, Bull. Amer. Math. Soc. 84 (1978), 886-912.

[Ho7] _ Cohen-Macaulay rings and modules, Proc. Internat. Congr. Math., Helsinki, Finland, Vol. I, Academia Scientarium Fennica, 1980, pp. 291-298.

[Ho8] __ Associated graded rings derived from integrally closed ideals, Proc. Conf. Commutative Algebra (Rennes, France, May 1981), pp. 1-27.

[Ho9] ___ Canonical elements in local cohomology modules and the direct summand conjecture, J. Algebra 84 (1983), 503-553. 
[HoE] M. Hochster and J. A. Eagon, Cohen-Macaulay rings, invariant theory, and the generic perfection of determinantal loci, Amer. J. Math. 93 (1971), 1020-1058.

[HR1] M. Hochster and J. L. Roberts, Rings of invariants of reductive groups acting on regular rings are Cohen-Macaulay, Adv. in Math. 13 (1974), 115-175.

[HR2] _ The purity of the Frobenius and local cohomology, Adv. in Math. 21 (1976), 117-172.

[Hu1] C. Huneke, Hilbert functions and symbolic powers, Michigan Math. J. 34 (1987), 293-318.

[Hu2] _ An algebraist commuting in Berkeley, Math. Intelligencer 11 (1989), 40-52.

[It1] S. Itoh, Integral closures of ideals generated by regular sequences, J. Algebra 117 (1988), 390-401.

[It2] _ Integral closures of ideals of the principal class, Hiroshima Math. J. 17 (1987), 373375.

[Kap] I. Kaplansky, Commutative algebra, Allyn and Bacon, 1970; revised edition, Univ. Chicago Press, Chicago, IL, 1974.

[Ke] G. Kempf, The Hochster-Roberts theorem of invariant theory, Michigan Math. J. 26 (1979), 19-32.

[Ku1] E. Kunz, Characterizations of regular local rings of characteristic p, Amer. J. Math. 91 (1969), $772-784$.

[Ku2] _ On Noetherian rings of characteristic p, Amer. J. Math. 98 (1976), 999-1013.

[L] J. Lipman, Relative Lipschitz saturation, Amer. J. Math. 97 (1975), 791-813.

[LS] J. Lipman and A. Sathaye, Jacobian ideals and a theorem of Briançon-Skoda, Michigan Math. J. 28 (1981), 199-222.

[LT] J. Lipman and B. Teissier, Pseudo-rational local rings and a theorem of Briançon-Skoda about integral closures of ideals, Michigan Math. J. 28 (1981), 97-116.

[Ma] F. Ma, Splitting in integral extensions, Cohen-Macaulay modules and algebras, J. Algebra 116 (1988), 176-195.

[Mac] S. Mac Lane, Homology, Springer-Verlag, Berlin, Gottingen, and Heidelberg, 1963.

[Mat] H. Matsumura, Commutative algebra, Benjamin, New York, 1970.

[MeSr] V. B. Mehta and V. Srinivas, Normal F-pure surface singularities, Tata Institute, Bombay, preprint.

[Mon] P. Monsky, The Hilbert-Kunz function, Math. Ann. 263 (1983), 43-49.

[N] M. Nagata, Local rings, Interscience, New York, 1972.

[NoR1] D. G. Northcott and D. Rees, Reductions of ideals in local rings, Proc. Cambridge Philos. Soc. 50 (1954), 145-158.

[NoR2] _ A note on reductions of ideals with an application to the generalized Hilbert function, Proc. Cambridge Philos. Soc. 50 (1954), 353-359.

[PS1] C. Peskine and L. Szpiro, Dimension projective finie et cohomologie locale, Inst. Hautes Études Sci. Publ. Math. 42 (1973), 323-395.

[PS2] _ Syzygies et multiplicités, C. R. Acad. Sci. Paris Sér. A 278 (1974), 1421-1424.

[Rat] L. J. Ratliff, Chain conjectures in ring theory, Springer-Verlag, Berlin and New York, 1978.

[Re1] D. Rees, $A$ note on asymptotically equidimensional ideals, Math. Proc. Cambridge Philos. Soc. 98 (1985), 33-35.

[Re2] — Reductions of modules, Math. Proc. Cambridge Philos. Soc. 101 (1987), 431-449.

[Ro1] P. Roberts, Two applications of dualizing complexes over local rings, Ann. Sci. École Norm. Sup. (4) 9 (1976), 103-106.

[Ro2] Cohen-Macaulay complexes and an analytic proof of the new intersection conjecture, J. Algebra 66 (1980), 225-230.

[Ro3] - Homological invariants of modules over commutative rings, Sém. Math. Sup., Presses Univ. Montréal, 1980.

[Ro4] _ The vanishing of intersection multiplicities of perfect complexes, Bull. Amer. Math. Soc. 13 (1985), 127-130. 
[Ro5] —, Le théorème d'intersection, C. R. Acad. Sci. Paris Sér I Math. 304 (1987), 177-180.

[Ro6] __, Intersection theorems, Commutative Algebra, Proc. Microprogram, June 15-July 12, 1987, Math. Sci. Res. Inst. Publ., no. 15, Springer-Verlag, New York, Berlin, Heidelberg, London, Paris, Tokyo, 1989, pp. 417-436.

[S] J.-P. Serre, Algèbre locale. Multiplicités, Lecture Notes in Math., no. 11, Springer-Verlag, Berlin, Heidelberg, and New York, 1965.

[Se] G. Seibert, Complexes with homology of finite length and Frobenius functors, preprint.

[Sk] H. Skoda, Applications des techniques $L^{2}$ a la théorie des idéaux d'une algèbre de fonctions holomorphes avec poids, Ann. Sci. Ecole Norm. Sup (4) 5 (1972), 545-579.

[Sr] V. Srinivas, Normal surface singularities of F-pure type, Tata Institute, Bombay, preprint.

[Sz] L. Szpiro, Sur la théorie des complexes parfaits, Commutative Algebra, Durham, 1981, London Math. Soc. Lecture Notes Ser., no 72, Cambridge Univ. Press, 1982, pp. 83-90.

[Tay] D. Taylor, Thesis, Ideals generated by monomials in an $R$-sequence, Univ. of Chicago, 1966.

[Wal] C. T. C. Wall, Lectures on $C^{\infty}$ stability and classification, Proc. Liverpool SingularitiesSymposium I, Lecture Notes in Math., no. 192, Springer-Verlag, Heidelberg, 1971.

[W] K. Watanabe, Study of F-purity in dimension two, Algebraic Geometry and Commutative Algebra in honor of Masayoshi Nagata, North-Holland, Amsterdam, New York, Oxford, 1987, pp. 791-800.

[ZS] O. Zariski and P. Samuel, Commutative algebra, Vols. I and II, Van Nostrand, Princeton, 1958 and 1960.

Department of Mathematics, University of Michigan, Ann Arbor, Michigan 481091003

Department of Mathematics, Purdue University, West Lafayette, Indiana 47907 